\title{
CRITICAL POINTS AND SUPERSYMMETRIC VACUA, III: STRING/M MODELS
}

\author{
MICHAEL R. DOUGLAS, BERNARD SHIFFMAN, AND STEVE ZELDITCH
}

\begin{abstract}
A fundamental problem in contemporary string/M theory is to count the number of inequivalent vacua satisfying constraints in a string theory model. This article contains the first rigorous results on the number and distribution of supersymmetric vacua of type IIb string theories compactified on a Calabi-Yau 3-fold $X$ with flux. In particular, complete proofs of the counting formulas in Ashok-Douglas [AD] and Denef-Douglas [DD1] are given, together with van der Corput style remainder estimates.

Supersymmetric vacua are critical points of certain holomorphic sections (flux superpotentials) of a line bundle $\mathcal{L} \rightarrow \mathcal{C}$ over the moduli space of complex structures on $X \times T^{2}$ with respect to the Weil-Petersson connection. Flux superpotentials form a lattice of full rank in a $2 b_{3}(X)$-dimensional real subspace $\mathcal{S} \subset H^{0}(\mathcal{C}, \mathcal{L})$. We show that the density of critical points in $\mathcal{C}$ for this lattice of sections is well approximated by Gaussian measures of the kind studied in DSZ1, DSZ2, AD, DD1.
\end{abstract}

Contents

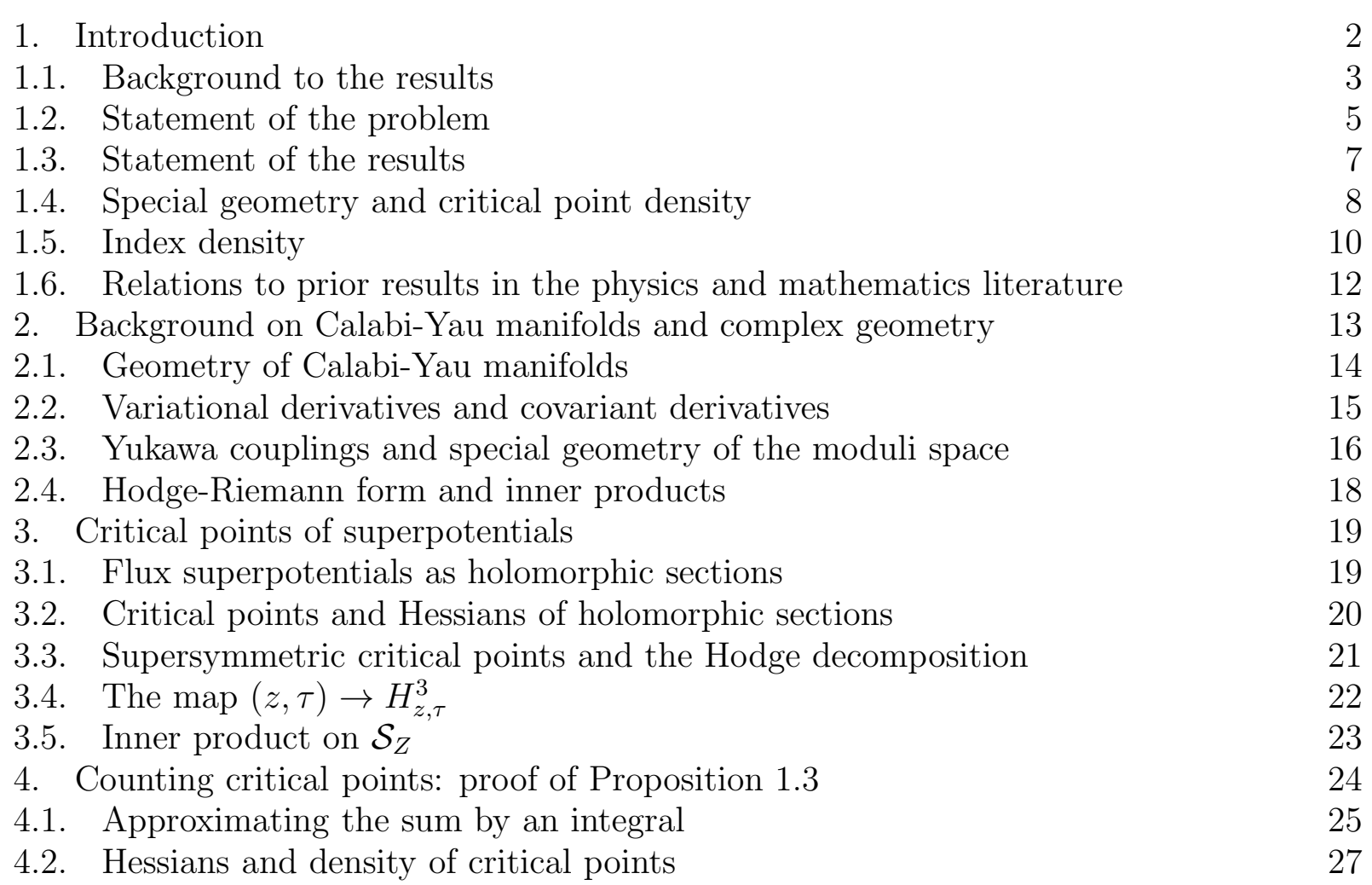

Research partially supported by DOE grant DE-FG02-96ER40959 (first author) and NSF grants DMS0100474 (second author) and DMS-0302518 (third author). 
4.3. Completion of the proof of Lemma 4.3 28

5. Proof of Theorem 1.4 29

5.1. A local van der Corput Theorem 30

5.2. Van der Corput for critical points 35

6. Special geometrv and density of critical points 36

6.1. The range of the Hessian map 36

6.2. Distortion of inner product under the Hessian map 38

6.3. Proof of Theorem 1.8 40

6.4. Examples 42

7. Problems and heuristics on the string theorv landscape 44

7.1. Complexity of the string theory landscape 44

7.2. Problems 45

7.3. Heuristic estimate of the critical point density 47

$\begin{array}{ll}\text { References } & 50\end{array}$

\section{INTRODUCTION}

This is the third in a series of articles DSZ1, DSZ2] (see also [Ze2]) by the authors on statistics of critical points of random holomorphic sections and their applications to the vacuum selection problem in string/M theory. We recall that, in these articles, a 'vacuum' in string theory is a Calabi-Yau manifold of complex dimension $d=3$ which forms the 6 'small dimensions' of the 10-dimensional universe, together with a choice of orientifolding and flux. Mathematically, vacua are critical points of a superpotential $W$, a holomorphic section of a line bundle $\mathcal{L} \rightarrow \mathcal{C}$ over the configuration space $\mathcal{C}$ which will be recalled in $\$ 1.1$ The 'vacuum selection problem' is that there exists no principle at present which selects a unique superpotential, nor a unique critical point of a given superpotential, out of a large ensemble of possible vacua. This motivates the program of studying statistics of vacua, whose basic problems are to count the number of vacua satisfying physically natural constraints and to determine how they are distributed in $\mathcal{C}$ (see [Do, DD1, AD, DGKT, KL, Si]). In this article, we present the first rigorous results on counting vacua with remainder estimates. In particular, we justify and improve on the approximations made in [DD1].

Our previous articles [DSZ1, DSZ2 were devoted to the statistics of critical points of Gaussian random holomorphic sections of line bundles over complex manifolds. The principal issue we face in this article is that the physically relevant ensembles of superpotentials are not Gaussian but rather are discrete ensembles of 'quantized flux' superpotentials which form a set of lattice points in a hyperbolic shell in $H^{3}(X, \mathbb{C})$. This hyperbolic shell is defined by the inequality (known as the tadpole constraint)

$$
0 \leq Q[\varphi] \leq L
$$

where

$$
Q[\varphi]=Q(\varphi, \bar{\varphi})=-\sqrt{-1} \int_{X} \varphi \wedge \bar{\varphi}
$$

is the Hodge-Riemann bilinear form. As will be recalled in $\$ 2.4, Q$ is an indefinite quadratic form, whose 'null cone' $\{G: Q[G]=0\}$ is a real quadric hypersurface which separates $H^{3}(X, \mathbb{C})$ into the interior $\{W: Q[G]>0\}$ and the exterior where $Q[G]<0$. As will be seen 
below (Propositions 3.1 and 2.11), only flux superpotentials corresponding to lattice points in $\{G: Q[G]>0\}$ contribute vacua, and that is why we consider the shell (11).

Our main results show that as $L \rightarrow \infty$, the statistics of critical points relative to the discrete lattice ensemble is well approximated by the statistics of critical points relative to the continuum ensemble in the shell, which is dual to the Gaussian ensembles of [DSZ1, DSZ2 and is therefore well understood. Thus, the vacuum statistics problem in string/M theory is a mixture of two kinds of equidistribution problems:

(1) The distribution of radial projections of lattice points onto a quadric hypersurface;

(2) The distribution of critical points of a continuous ensemble of random holomorphic sections (related to a Gaussian ensemble) of a negative line bundle, and their interpretation in the special geometry of Calabi-Yau moduli spaces.

The equidistribution problem in (2) is analyzed in detail in DSZ1, DD1, so the main purpose of this paper is to analyze (1) and to combine it with the previous analysis of (2).

At the end of this article in $\$ 7$ and in [Ze2], we compare the mathematical results of this article to discussions of vacua in the string theory literature.

1.1. Background to the results. To state our results, we will need some notation (see $§ 2$ for more details). The models we consider in this article are called type IIb flux compactifications GVW, GKP. We fix a complex 3-dimensional Calabi-Yau manifold $X$, i.e. a complex manifold with trivial canonical bundle $K_{X} \simeq \mathcal{O}$ and with first Betti number $b_{1}(X)=0$. In some of the physics literature, it is also assumed that $H^{2,0}(X)=0$, but our results hold without this assumption. For each complex structure $z$ on $X$, there is a corresponding Hodge decomposition

$$
H^{3}(X, \mathbb{C})=H_{z}^{3,0}(X) \oplus H_{z}^{2,1}(X) \oplus H_{z}^{1,2}(X) \oplus H_{z}^{0,3}(X) .
$$

The space $H_{z}^{3,0}(X)$ of $(3,0)$-forms relative to $z$ is one-dimensional and is spanned by a nowhere vanishing holomorphic volume form $\Omega_{z}$. We also put $b_{3}=b_{3}(X)=\operatorname{dim} H^{3}(X, \mathbb{R})$, $h^{p, q}=h^{p, q}(X)=\operatorname{dim}_{\mathbb{C}} H^{p, q}(X)$. Thus, $b_{3}=2\left(h^{2,1}+1\right)$.

When we speak of vacua of string theory compactified on the Calabi-Yau space $X$, we refer to classical vacua of the effective supergravity theory it determines. As discussed in St2, the effective supergravity Lagrangian is derived by 'integrating out' or neglecting the massive modes (positive eigenvalues) of various operators. The data of effective supergravity consists of $(\mathcal{C}, \mathcal{L}, W)$ where:

(1) $\mathcal{C}$ is the configuration space;

(2) $\mathcal{L} \rightarrow \mathcal{C}$ is a holomorphic line bundle.

(3) the superpotential $W$ is a holomorphic section of $\mathcal{L}$.

In type IIb flux compactifications the configuration space is the moduli space of Calabi-Yau (Ricci flat Kähler) product metrics on $X \times T^{2}$. At this time of writing, the study of vacua in string theory is simplified by replacing the moduli space of Calabi-Yau metrics by the moduli space of complex structures on $X$ (see e.g. [Do, $\mathrm{AD}$ ). In the case where $h^{2,0}(X)=0$, this is equivalent to fixing the Kähler class $[\omega] \in H^{2}(X, \mathbb{R})$ of the Calabi-Yau metrics. Hence we define the configuration space to be

$$
\mathcal{C}=\mathcal{M} \times \mathcal{E}
$$


where $\mathcal{M}$ is the moduli space of complex structures on $X$ and where $\mathcal{E}=\mathcal{H} / S L(2, \mathbb{Z})$ is the moduli space of elliptic curves. Throughout this paper we identify $\mathcal{C}=\mathcal{M} \times \mathcal{E}$ with a fundamental domain $\mathcal{D}$ for the modular group $\Gamma$ in the Teichmüller space $\mathcal{T}$ eich $(X) \times \mathcal{H}$ of complex structures (see \$2.1). For simplicity of exposition, we refer to restrictions to $\mathcal{D}$ of holomorphic objects on $\mathcal{T}$ eich $(X) \times \mathcal{H}$ as holomorphic objects over $\mathcal{C}$.

The line bundle $\mathcal{L}$ is defined to be the dual line bundle to the Hodge bundle $H^{3,0}(X) \otimes$ $H^{1,0}\left(T^{2}\right) \rightarrow \mathcal{C}$, where $T^{2}=\mathbb{R}^{2} / \mathbb{Z}^{2}$. We give $\mathcal{C}$ the Weil-Petersson Kähler form $\omega_{W P}$ induced from the Weil-Petersson metric on $\mathcal{L}$ (see $\$ 3.3)$. To be precise, $\mathcal{L}$ is a holomorphic line bundle over $\mathcal{T}$ eich $(X) \times \mathcal{H}$, and $W$ is a holomorphic section of $\mathcal{T}$ eich $(X) \times \mathcal{H}$. But as mentioned above, by holomorphic sections $W \in H^{0}(\mathcal{C}, \mathcal{L})$ we mean restrictions to $\mathcal{D}$ of holomorphic sections of $H^{0}(\mathcal{T}$ eich $(X) \times \mathcal{H}, \mathcal{L})$.

Type IIb flux compactifications contain two non-zero harmonic 3-forms $F, H \in H^{3}(X, \mathbb{Z})$ which are known respectively as the RR (Ramond-Ramond) and NS (Neveu-Schwartz) 3form field strengths. We combine them into a complex flux $G=F+i H \in H^{3}(X, \mathbb{Z} \oplus i \mathbb{Z})$. The parameter $\tau \in \mathcal{E}$ is known as the dilaton-axion and may be viewed as the period of $\omega_{\tau}=d x+\tau d y$ over the one-cycle dual to $d y$ in $T^{2}$. Given $G \in H^{3}(X, \mathbb{Z} \oplus \sqrt{-1} \mathbb{Z})$, physicists define the corresponding flux superpotential $W_{G}$ by:

$$
W_{G}(z, \tau)=\int_{X}(F+\tau H) \wedge \Omega_{z}
$$

where $\Omega_{z} \in H^{3,0}(X)$. This is not well-defined as a function on $\mathcal{C}$ since $\Omega_{z}$ and $\tau$ depend on a choice of frame. To be more precise, $G \in H^{3}(X, \mathbb{C})$ determines a section $W_{G}$ of the line bundle

$$
\mathcal{L}=\left(H^{3,0}(X) \otimes H^{1,0}\left(T^{2}\right)\right)^{*} \rightarrow \mathcal{T} \operatorname{eich}(X) \times \mathcal{H}
$$

by making $G$ into the following linear functional on $H_{z}^{3,0}(X) \otimes H_{\tau}^{1,0}\left(T^{2}\right)$ :

$$
\left\langle W_{G}(z, \tau), \Omega_{z} \otimes \omega_{\tau}\right\rangle=\int_{X \times T^{2}}(F \wedge d y-H \wedge d x) \wedge\left(\Omega_{z} \wedge \omega_{\tau}\right) .
$$

The map $G \rightarrow W_{G}$ defines an injective real (but not complex) linear map which embeds complex integral fluxes

$$
H^{3}(X, \mathbb{Z} \oplus \sqrt{-1} \mathbb{Z}) \rightarrow H^{0}(\mathcal{C}, \mathcal{L})
$$

as a lattice of rank $2 b_{3}$ in $H^{0}(\mathcal{M} \times \mathcal{E}, \mathcal{L})$ which we call the lattice $\mathcal{S}^{\mathbb{Z}}$ of integral flux superpotentials. The real span

$$
\mathcal{S}=\mathbb{R} \mathcal{S}^{\mathbb{Z}} \subset H^{0}(\mathcal{M}, \mathcal{L})
$$

of $\mathcal{S}^{\mathbb{Z}}$ is also important, and will be referred as the space of flux superpotentials. We emphasize here that $\mathcal{S}$ is not a complex vector space, nor are any of the associated spaces discussed below. We also use the (real-linear) map $G \mapsto W_{G}$ to regard $Q$ as a quadratic form on $\mathcal{S}$, writing

$$
Q\left[W_{G}\right]:=Q[G]=-\sqrt{-1} \int_{X} G \wedge \bar{G}=2 \int_{X} F \wedge H, \quad G=F+i H \in H^{3}(X, \mathbb{C}) .
$$

The bundles $H_{z}^{3,0} \rightarrow \mathcal{M}$ and $H_{\tau}^{1,0} \rightarrow \mathcal{E}$ carry Weil-Petersson Hermitian metrics $h_{W P}$ defined by

$$
h_{W P}\left(\Omega_{z}, \Omega_{z}\right)=e^{-K(z, \bar{z})}=i \int_{X} \Omega_{z} \wedge \bar{\Omega}_{z}
$$


and their associated Chern connections $\nabla_{W P}$. They induce dual metrics and connections on $\mathcal{L}$. We denote the connection simply by $\nabla$.

1.2. Statement of the problem. Given a flux superpotential $W$, there is an associated potential energy on $\mathcal{C}$ defined by

$$
V_{W}(Z)=|\nabla W(Z)|^{2}-3|W(Z)|^{2} .
$$

(See WB for background on $V$ ). By a vacuum we mean a critical point of $V(Z)$ on $\mathcal{C}$. In this paper, we only study supersymmetric vacua, namely $Z \in \mathcal{C}$ which are connection critical points in the sense that $\nabla_{W P} W(Z)=0$. We denote the set of supersymmetric vacua of $W$ by

$$
\operatorname{Crit}(W)=\left\{Z \in \mathcal{C}: \nabla_{W P} W(Z)=0\right\}
$$

Our goal is thus to count and find the distribution law of the supersymmetric vacua

$$
\{\text { SUSY vacua }\}=\bigcup_{G \in \mathcal{S}^{\mathbb{Z}}: Q[G] \leq L} \operatorname{Crit}\left(W_{G}\right)
$$

as $W_{G}$ varies over the lattice $\mathcal{S}^{\mathbb{Z}}$ within the hyperbolic shell (11). To define the distribution law, we introduce the incidence relation

$$
\mathcal{I}=\left\{\left(W_{G}, Z\right) \in \mathcal{S} \times \mathcal{C}: \nabla W_{G}(Z)=0\right\} .
$$

We shall view $\mathcal{C}$ as a fundamental domain for the modular group $\Gamma$ in Teichmüller space (cf. \$2). The incidence variety $\mathcal{I}$ is then a real $2 m$-dimensional subvariety of $\mathcal{C} \times \mathcal{S}$ with the following diagram of projections:

$$
\begin{array}{ll} 
& \multicolumn{1}{|c}{\subset \mathcal{C} \times \mathcal{S}} \\
\rho \swarrow & \searrow \pi \\
\mathcal{C} & \mathcal{S}
\end{array}
$$

The fiber $\pi^{-1}(W)$ is the set $\operatorname{Crit}(W)$ of critical points of $W$ in $\mathcal{C}$. Since $\mathcal{C}$ is regarded as a fundamental domain in Teichmüller space, the map $\pi$ is not surjective: there exist $W$ with no critical points in $\mathcal{C}$; hence $\pi(\mathcal{C})$ is a domain with boundary in $\mathcal{S}$ (see 66.4.1). Critical points can move out of $\mathcal{C}$ as $W$ varies in $\mathcal{S}$. (There is a similar but more complicated theory of non-supersymmetric vacua [DD2.)

The fibers of $\rho$ are the subspaces

$$
\mathcal{S}_{Z}:=\left\{W \in \mathcal{S}: \nabla_{W P} W(Z)=0\right\},
$$

which play a crucial role in this article. They have the remarkable Hodge theoretic identifications,

$$
\mathcal{S}_{z, \tau} \equiv H_{z}^{2,1}(X) \oplus H_{z}^{0,3}(X) \quad \text { (Proposition 3.1) }
$$

It then follows (see Proposition [3.2) that $\mathcal{I} \stackrel{\rho}{\rightarrow} \mathcal{C}$ is a vector bundle (with fiber $\approx \mathbb{C}^{b_{3} / 2}$ ) over a manifold with boundary. Another key point is that the restrictions of $Q$ to the fibers are always positive definite:

$$
\left.Q\right|_{H_{z}^{2,1}(X) \oplus H_{z}^{0,3}(X)} \gg 0 \quad \text { (Proposition 2.1), }
$$

i.e. $\mathcal{S}_{Z}$ lies in the positive cone $\{Q(\varphi, \bar{\varphi})>0\}$ of the indefinite quadratic (Hodge-Riemann) form (2) (cf. 92.4). 
We now define the discriminant locus

$$
\widetilde{\mathcal{D}}=\left\{(Z, W) \in \mathcal{I}: \operatorname{det} H^{c} W(Z)=0\right\}
$$

of points $(Z, W) \in \mathcal{I}$ such that $Z$ is a degenerate critical point of $W$, where $H^{c} W(Z)$ is the complex Hessian of $W$ at the critical point $Z$ as defined in (59)-(61). Equivalently, $\widetilde{\mathcal{D}}$ is the set of critical points of the second projection $\mathcal{I} \stackrel{\pi}{\rightarrow} \mathcal{S}$ together with the singular points of $\mathcal{I}$. Its image $\mathcal{D}=\pi(\widetilde{\mathcal{D}})$ under $\pi$ is the discriminant variety of superpotentials with degenerate critical points.

For each $W \in \mathcal{S} \backslash\{0\}$, we define its distribution of (non-degenerate) critical points as the measure $C_{W}$ on $\mathcal{I} \backslash \widetilde{\mathcal{D}}$ defined by

$$
\left\langle C_{W}, \psi\right\rangle=\sum_{Z \in C r i t(W)} \psi(Z, W),
$$

for $\psi \in \mathcal{C}(\mathcal{I})$ such that $\rho(\operatorname{Supp} \psi)$ is relatively compact in $\mathcal{C}$ and $\operatorname{Supp} \psi$ is disjoint from $\widetilde{\mathcal{D}}$. A more general definition of $C_{W}$ is

$$
C_{W}=\left|\operatorname{det} H^{c} W(Z)\right| \nabla W^{*} \delta_{0}
$$

which will be discussed in 4.2 . We make these assumptions on $\psi$ so that the sum on the right side is a finite and well-defined sum. Indeed, the pull back is not well-defined (without further work) on $\widetilde{\mathcal{D}}$. We will say more about $\widetilde{\mathcal{D}}$ after the statement of Theorem 1.4.

The basic sums we study are :

$$
\begin{aligned}
\mathcal{N}_{\psi}(L) & =\sum\left\{\left\langle C_{N}, \psi\right\rangle: N \in \mathcal{S}^{\mathbb{Z}}, Q[N] \leq L\right\} \\
& =\sum\left\{\psi(Z, N):(Z, N) \in \mathcal{I}, N \in \mathcal{S}^{\mathbb{Z}}, 0 \leq Q[N] \leq L\right\} .
\end{aligned}
$$

For instance, when $\psi \equiv \chi_{K}$ is the characteristic function of a compact subset $K \subset \subset \mathcal{I} \backslash \widetilde{\mathcal{D}}$, $N_{\psi}(L)$ counts the total number of non-degenerate critical points lying over $\rho(K)$ coming from all integral flux superpotentials with $Q[W] \leq L$. Physicists are naturally interested in counting the number of vacua with close to the observed values of the cosmological constant and other physical quantities, and hence would study sums relevant to such quantities. For instance, the cosmological constant of the theory defined by a vacuum $Z$ is the value $V(Z)$ of the potential there (see [DD1], §3.3). Thus, we may state the main problem of this paper:

Problem 1.1. Find the asymptotics and remainder for $\mathcal{N}_{\psi}(L)$ as $L \rightarrow \infty$.

As indicated above, this problem is very closely related to the pure lattice point problem of measuring the rate of uniform distribution of radial projections of lattice points onto the surface of a quadric hypersurface. More generally one could consider any smooth strictly convex set $Q \subset \mathbb{R}^{n}(n \geq 2)$ with $0 \in Q^{\circ}$. Associated to $Q$ is the norm $|X|_{Q}$ of $X \in \mathbb{R}^{n}$ defined by

$$
Q=\left\{X \in \mathbb{R}^{n}:|X|_{Q}<1\right\} .
$$

To measure the equidistribution of radial projections of lattice points to $\partial Q$, one considers the sums

$$
S_{f}(t)=\sum_{k \in \mathbb{Z}^{n} \cap t Q \backslash\{0\}} f\left(\frac{k}{|k|_{Q}}\right), \quad \text { with } f \in C^{\infty}(\partial Q), t>0 .
$$

The parallel lattice point problem is then 
Problem 1.2. Find the asymptotics and remainder for $S_{f}(t)$ as $t \rightarrow \infty$.

1.3. Statement of the results. In Theorem 5.1 we obtain a van der Corput type estimate for the lattice point problem 1.2. For the critical point problem, we first give an elementary formula which is based on a trivial lattice counting estimate (which is useful since it is sometimes sharp), namely where the remainder term is simply a count of the cubes of the lattice which intersect the boundary. We denote by $\chi_{Q_{Z}}$ the characteristic function of the shell $\left\{W \in \mathcal{S}_{Z}: 0<Q_{Z}[W]<1\right\}$.

Proposition 1.3. Suppose that $\psi=\chi_{K}$ where $K \subset \mathcal{I}$ such that $(Z, W) \in K \Leftrightarrow(Z, r W) \in$ $K$ for $r \in \mathbb{R}^{+}$. Assume further that $\rho(K)$ is relatively compact in $\mathcal{C}$ and $\pi(\partial K)$ is piecewise smooth. Then

$$
\mathcal{N}_{\psi}(L)=L^{b_{3}}\left[\int_{\mathcal{C}} \int_{\mathcal{S}_{Z}} \psi(Z, W)\left|\operatorname{det} H^{c} W(Z)\right| \chi_{Q_{Z}}(W) d W d \operatorname{Vol}_{W P}(Z)+O\left(L^{-1 / 2}\right)\right] .
$$

Here and in Theorem 1.4 below, $d W$ means the multiple of Lebesgue measure on $\mathcal{S}_{Z}$ which gives the volume form for the positive-definite quadratic form $Q_{Z}=\left.Q\right|_{\mathcal{S}_{Z}}$. We note that the integral converges, since by (18), $\left\{Q_{Z} \leq 1\right\}$ is an ellipsoid of finite volume.

It would be interesting to know if the remainder estimate is sharp for any domain $K \subset \mathcal{I}$. In the pure lattice point Problem 1.2, the corresponding 'trivial estimate' is sharp. For instance, consider the domain $K=S_{+}^{n-1} \subset S^{n-1}$ formed by the northern hemisphere and put $\psi=\chi_{K}$. Then the remainder term

$$
\sum_{k \in \mathbb{Z}^{n},|k| \leq \sqrt{L}} \chi_{K}\left(\frac{k}{|k|}\right)-L^{\frac{n}{2}} \int_{K} f d A
$$

reflects the concentration of projections of lattice points on the boundary $\partial S_{+}^{n-1}$, namely a great equatorial sphere. When the equator is defined by $x_{n}=0$, the lattice points projecting over the equator are the lattice points in $\mathbb{Z}^{n-1} \subset \mathbb{R}^{n-1}$ and the number with $|k| \leq \sqrt{L}$ is of size $\sim L^{\frac{n-1}{2}}$. Analogously one may ask if there are domains $K \subset \mathcal{C}$ along which critical points concentrate to the same maximal degree. Some evidence that the answer is 'no' will be presented in \$4.1.

Our main result stated below is a much sharper van der Corput type asymptotic estimate of $\mathcal{N}_{\psi}(L)$ as $L \rightarrow \infty$ for homogeneous test functions which vanish near the discriminant locus. Here, we say that a function $\psi \in \mathcal{C}(\mathcal{I})$ is homogeneous of order $\alpha$ if

$$
\psi(Z, r W)=r^{\alpha} \psi(Z, W), \quad(Z, W) \in \mathcal{I}, r \in \mathbb{R}^{+} .
$$

We consider homogeneous functions since they include (smoothed) characteristic functions as well as the cosmological constant (which is homogeneous of degree 2).

ThEOREM 1.4. Let $\psi \in \mathcal{C}^{\infty}(\mathcal{I})$ be homogeneous of order $\alpha \geq 0$ and suppose that $\rho(\operatorname{Supp} \psi)$ is a compact subset of $\mathcal{C}$ and $\operatorname{Supp} \psi \cap \widetilde{\mathcal{D}}=\emptyset$. Then

$$
\mathcal{N}_{\psi}(L)=L^{b_{3}+\alpha / 2}\left[\int_{\mathcal{C}} \int_{\mathcal{S}_{Z}} \psi(Z, W)\left|\operatorname{det} H^{c} W(Z)\right| \chi_{Q_{Z}}(W) d W d \operatorname{Vol}_{W P}(Z)+O\left(L^{-\frac{2 b_{3}}{2 b_{3}+1}}\right)\right] .
$$

It is reasonable to make the assumption $\operatorname{Supp} \psi \cap \widetilde{\mathcal{D}}=\emptyset$, because degenerate critical points cannot be physically acceptable vacua in string/M theory. Indeed, the Hessian of $W$ at a critical point defines the 'fermionic mass matrix' of the theory, and a degenerate critical 
point would give rise to massless fermions which are not observed in physics. (See WB for definitions of the mass matrix.)

Let us note some key features of the geometry of $\widetilde{\mathcal{D}}$ which play a role in the assumptions (and proofs) of Proposition 1.3] and Theorem [1.4. First, as observed in [DSZ1, DSZ2, its defining equation

$$
\operatorname{det} H^{c} W(Z)=\operatorname{det}\left(H^{*} H-|W|^{2} I\right)=0
$$

is real valued; here, $H$ is the holomorphic Hessian (see 33.2 ). Hence, $\widetilde{\mathcal{D}} \subset \mathcal{I}$ is a real analytic hypersurface (with boundary). For test functions $\psi$ which do not vanish on $\widetilde{\mathcal{D}}$, the expression $\left\langle C_{W}, \psi\right\rangle$ (when well-defined) can jump as one passes from one component of $\mathcal{S} \backslash \mathcal{D}$ to another or across the boundary of $\mathcal{C}$. It follows from (23) that $\widetilde{\mathcal{D}} \cap\left(\{Z\} \times \mathcal{S}_{Z}\right)$ is a real conic hypersurface for all $Z \in \mathcal{C}$. Thus $\widetilde{\mathcal{D}} \rightarrow \mathcal{C}$ is a bundle of conic hypersurfaces and $\rho(\widetilde{\mathcal{D}})=\mathcal{C}$; i.e., every point of moduli space is a degenerate critical point of some superpotential. We further note that $\mathcal{S} \backslash \mathcal{D}$ consists of a finite number of connected components, and that $\pi: \mathcal{I} \backslash \widetilde{\mathcal{D}} \rightarrow \pi(\mathcal{S}) \backslash \mathcal{D}$ is a finite covering over each connected component of $\pi(\mathcal{S}) \backslash \mathcal{D}$.

1.4. Special geometry and critical point density. In obtaining reliable order of magnitude results on numbers of vacua in a given string/M model, it is important to estimate the size of the leading coefficient

$$
\int_{\mathcal{C}} \psi(Z) \int_{\mathcal{S}_{Z}}\left|\operatorname{det} H^{c} W(Z)\right| \chi_{Q_{Z}}(W) d W d \operatorname{Vol}_{W P}(Z)
$$

and of the remainder. Since little is known about the volume of $\mathcal{C}$ at present (cf. [LS1]), we concentrate on estimating the integrand

$$
\mathcal{K}^{\mathrm{crit}}(Z):=\int_{\mathcal{S}_{Z}}\left|\operatorname{det} H^{c} W(Z)\right| \chi_{Q_{Z}} d W
$$

in the $b_{3}$ aspect. It is also important to study the behavior of the $\mathcal{K}^{\text {crit }}(Z)$ as $Z$ tends to 'infinity' in $\mathcal{C}$, or to a singular point such as a conifold point (when one exists).

A key feature of $\mathcal{K}^{\text {crit }}(Z)$ is that it is the integral of a homogeneous function of order $b_{3}$ over a space of dimension $\operatorname{dim}_{\mathbb{R}} \mathcal{S}_{Z}=b_{3}=2\left(h^{2,1}+1\right)$. Among the known Calabi-Yau 3-folds it is common to have $300<b_{3}<1000$, hence the integral is often over a space of large dimension. The $b_{3}$-dependence is sensitive since (e.g.) the ratio of the $L^{\infty}$ norm to the $L^{2}$ norm of a homogeneous function of degree $b_{3}$ in $b_{3}$ variables can be of order $b_{3}^{b_{3}}$. It is useful to have alternative formulas for the leading coefficient, and we now present a few. We will use them to suggest conjectures on the order of magnitude of $\mathcal{K}^{\text {crit }}(Z)$ in the $b_{3}$ aspect in $\$ 7$

First, using the homogeneity of the integrand, we may rewrite the integral in terms of a Gaussian density

$$
\mathcal{K}^{\text {crit }}(Z)=\frac{1}{b_{3} !} \int_{\mathcal{S}_{Z}}\left|\operatorname{det} H^{c} W(Z)\right| e^{-\left\langle Q_{Z} W, W\right\rangle} d W
$$

This formula shows that $\mathcal{K}^{\text {crit }}$ is formally analogous to density of critical points of random holomorphic sections relative to a Gaussian measure studied in [DSZ1. For this reason, we call (24) the critical point density. However, the measure $e^{-Q[W]} \chi_{\{0<Q<1\}}(W) d W$ is of infinite volume, so the analogy should not be taken too literally. The density $\mathcal{K}^{\text {crit }}(Z)$ is well-defined despite the infinite volume of the underlying measure on $\mathcal{S}$ because the fibers 
$Q_{Z}$ of $\left.\rho\right|_{Q}$ are of finite volume. Indeed, the conditional measures of $e^{-Q[W]} d W$ are standard (un-normalized) Gaussian measures $e^{-Q_{Z}(W)} d W$.

Next, we rewrite the integrals by the methods in [DSZ1, DSZ2. The first method is to change variables to the Hessian $H^{c} W(Z)$, i.e. to 'push-forward' the $\mathcal{S}_{Z}$ integral under the Hessian map

$$
H_{Z}: \mathcal{S}_{Z} \rightarrow \operatorname{Sym}(m, \mathbb{C}) \oplus \mathbb{C}, \quad H_{Z}(W)=H^{c} W(Z)
$$

where $m=\operatorname{dim} \mathcal{C}=h^{2,1}+1$. In [DSZ1, DSZ2, we used this change of variables to simplify the formulas for the density of critical points. There, however, the spaces of holomorphic sections of the line bundles $L \rightarrow M$ were so large that the image of the Hessian map was the entire space $\operatorname{Sym}(m, \mathbb{C}) \oplus \mathbb{C}$ of complex Hessians of rank equal to the dimension $m=\operatorname{dim} M$. In the case of type IIb flux compactifications, the dimension of the configuration space $\mathcal{C}$ is as large as the dimension of the space $\mathcal{S}$ of sections, and the Hessian map is by no means surjective. Indeed, in Lemma 6.1, we prove that the Hessian map is an isomorphism to a real $b_{3}$-dimensional space $\mathcal{H}_{Z} \oplus \mathbb{C}$, where $\mathcal{H}_{Z}$ is spanned (over $\mathbb{R}$ ) by the $2 h^{2,1}$ Hermitian matrices

$$
\xi^{j}:=\left(\begin{array}{cc}
0 & e_{j} \\
e_{j}^{t} & \mathcal{F}^{j}(z)
\end{array}\right), \quad \xi^{h^{2,1}+j}:=\left(\begin{array}{cc}
0 & i e_{j} \\
i e_{j}^{t} & -i \mathcal{F}^{j}(z)
\end{array}\right), \quad j=1, \ldots, h^{2,1} .
$$

Here, $e_{j}$ is the $j$-th standard basis element of $\mathbb{C}^{h^{2,1}}$ and $\mathcal{F}^{j}(z) \in \operatorname{Sym}\left(h^{2,1}, \mathbb{C}\right)$ is the matrix $\left(\mathcal{F}_{i k}^{\bar{j}}(z)\right)$ whose entries define the 'Yukawa couplings' on $\mathcal{M}$ (see (46), \$2.3] or [St1, [CO]) with respect to normal coordinates at the point $z \in \mathcal{M}$.

Since $\mathcal{H}_{Z}$ is not a complex subspace of $\operatorname{Sym}(m, \mathbb{C})$, we regard $\operatorname{Sym}(m, \mathbb{C})$ as a real vector space with inner product

$$
(A, B)_{\mathbb{R}}=\operatorname{Re}\langle A, B\rangle_{H S}=\operatorname{Re}\left(\operatorname{Trace} A B^{*}\right) .
$$

To state our next result, we let $\Lambda_{Z}$ be the operator given by the distortion under the Hessian map (see 6.2 ):

$$
\left(\left(\Lambda_{Z} \oplus I_{\mathbb{C}}\right)^{-1} H_{Z} W, H_{Z} W\right)_{\mathbb{R}}=Q[W] \quad\left(W \in \mathcal{S}_{Z}\right),
$$

where $Q[W]$ is given by (9). In terms of the basis $\left\{\xi^{a}\right\}_{1 \leq a \leq 2 h^{2,1}}$,

$$
\Lambda_{Z} \xi^{a}=\sum_{b=1}^{2 h^{2,1}} \Lambda_{a b} \xi^{b}, \quad \Lambda_{a b}=\left(\xi^{a}, \xi^{b}\right)_{\mathbb{R}} .
$$

The $\Lambda$ matrix has the block form

$$
\left(\Lambda_{a b}\right)=\left(\begin{array}{cc}
\Lambda^{\prime} & \Lambda^{\prime \prime} \\
\Lambda^{\prime \prime} & \Lambda^{\prime}
\end{array}\right), \quad \Lambda_{j k}^{\prime}=2 \delta_{j k}+\operatorname{Re} \operatorname{Tr} \mathcal{F}^{j} \mathcal{F}^{k *}, \quad \Lambda_{j k}^{\prime \prime}=\operatorname{Im} \operatorname{Tr} \mathcal{F}^{j} \mathcal{F}^{k *}
$$

In Proposition 6.2, we show that the $(1,1)$ form

$$
\omega_{\Lambda}:=\frac{i}{2} \sum\left(\Lambda_{j k}^{\prime}+i \Lambda_{j k}^{\prime \prime}\right) d z^{j} \wedge d \bar{z}^{k}=\frac{i}{2} \sum\left[2 \delta_{j k}+\operatorname{Tr} \mathcal{F}^{j}\left(z_{0}\right) \mathcal{F}^{k *}\left(z_{0}\right)\right] d z^{j} \wedge d \bar{z}^{k}
$$

is the so-called Hodge metric $(m+3) \omega_{W P}+\operatorname{Ric}\left(\omega_{W P}\right)$ of the Weil-Petersson metric [Lu, Wa2]. 
By the injectivity of the Hessian map (stated in Lemma 6.1), we can make the change of variables $W \mapsto(H, x)$ in (24) - (25) to obtain the following alternate formulas for $\mathcal{K}^{\text {crit }}(Z)$ :

$$
\begin{aligned}
\mathcal{K}^{\text {crit }}(Z) & =\left.\frac{1}{\sqrt{\operatorname{det} \Lambda_{Z}}} \int_{\mathcal{H}_{Z} \oplus \mathbb{C}}\left|\operatorname{det} H^{*} H-\right| x\right|^{2} I \mid \chi_{\Lambda_{Z}}(H, x) d H d x, \\
& =\left.\frac{1}{b_{3} ! \sqrt{\operatorname{det} \Lambda_{Z}}} \int_{\mathcal{H}_{Z} \oplus \mathbb{C}}\left|\operatorname{det} H^{*} H-\right| x\right|^{2} I \mid e^{-\left(\Lambda_{Z}^{-1} H, H\right)_{\mathbb{R}}-|x|^{2}} d H d x
\end{aligned}
$$

where $\chi_{\Lambda_{Z}}$ is the characteristic function of the ellipsoid $\left\{\left(\Lambda_{Z}^{-1} H, H\right)_{\mathbb{R}}+|x|^{2} \leq 1\right\}$. These formulas are analogous to Theorem 1 and Corollary 2 of [DSZ1, the key difference being that here we integrate over a moving subspace $\mathcal{H}_{Z}$ of symmetric matrices.

We similarly have the following alternative formulations of Proposition 1.3 and Theorem 1.4 .

Corollary 1.5. Let $\psi=\chi_{K}$, where $K \subset \mathcal{I}$ is as in Proposition 1.3, and let $\tilde{\psi}\left(Z, H_{Z} W\right)=$ $\psi(Z, W)$. Then,

$$
\begin{array}{r}
\mathcal{N}_{\psi}(L)=\frac{L^{b_{3}}}{b_{3} !}\left[\left.\int_{\mathcal{C}} \frac{1}{\sqrt{\operatorname{det} \Lambda_{Z}}} \int_{\mathcal{H}_{Z} \oplus \mathbb{C}} \tilde{\psi}(Z ; H, x)\left|\operatorname{det} H^{*} H-\right| x\right|^{2} I \mid e^{-\left(\Lambda_{Z}^{-1} H, H\right)_{\mathbb{R}}-|x|^{2}} d H d x d \operatorname{Vol}_{W P}(Z)\right. \\
\left.+O\left(L^{-1 / 2}\right)\right] .
\end{array}
$$

Corollary 1.6. Let $\psi \in \mathcal{C}^{\infty}(\mathcal{I})$ be homogeneous of order $\alpha \geq 0$ and suppose that $\rho(\operatorname{Supp} \psi)$ is a compact subset of $\mathcal{C}$ and $\operatorname{Supp} \psi \cap \widetilde{\mathcal{D}}=\emptyset$. Let $\tilde{\psi}\left(Z, H_{Z} W\right)=\psi(Z, W)$. Then,

$$
\begin{aligned}
\mathcal{N}_{\psi}(L)=\frac{L^{b_{3}+\alpha / 2}}{\Gamma\left(b_{3}+\alpha / 2+1\right)}\left[\int_{\mathcal{C}} \frac{1}{\sqrt{\operatorname{det} \Lambda_{Z}}} \int_{\mathcal{H}_{Z} \oplus \mathbb{C}} \tilde{\psi}(Z ; H, x)\right. \\
\left.\quad \times\left.\left|\operatorname{det} H^{*} H-\right| x\right|^{2} I \mid e^{-\left(\Lambda_{Z}^{-1} H, H\right)_{\mathbb{R}}-|x|^{2}} d H d x d \operatorname{Vol}_{W P}(Z)+O\left(L^{-\frac{2 b_{3}}{2 b_{3}+1}}\right)\right] .
\end{aligned}
$$

It is not obvious how to estimate the dependence of the integral for $\mathcal{K}^{\text {crit }}(Z)$ on the subspace $\mathcal{H}_{Z}$. There are two natural ways to parameterize this space. One (which is used in [DD1]) is to use as a basis of $\mathcal{H}_{Z}$ the Hessians of a $Q_{Z}$-orthonormal basis of $\mathcal{S}_{Z}$. A second method is to use the orthonormal basis of eigenmatrices $\left\{H_{j}\right\}$ of $\Lambda_{Z}$ with respect to the inner product (28). We thus put $\Lambda_{Z} H_{j}(Z)=\mu_{j}(Z) H_{j}(Z)$, and $H(y, Z)=\sum_{j} y_{j} H_{j}(Z)$. We also let $D(\mu)$ denote the diagonal matrix with entries $\mu_{j}$. Changing variables to $\mu_{j}^{1 / 2} y$ cancels $\frac{1}{\sqrt{\operatorname{det} \Lambda_{Z}}}$ and we obtain:

Corollary 1.7. We have:

$$
\mathcal{K}^{\text {crit }}(Z)=\left.\int_{|y|^{2}+|x|^{2} \leq 1}\left|\operatorname{det} H(D(\mu) y, Z)^{*} H(D(\mu) y, Z)-\right| x\right|^{2} I \mid d y d x .
$$

In $\$ 7$ we will discuss some conjectural bounds on the density of critical point based on the assumption that the subspaces $\mathcal{H}_{Z}$ are sufficiently random subspaces of $\operatorname{Sym}\left(h^{2,1}, \mathbb{C}\right)$.

1.5. Index density. The absolute value in the expressions for the distribution of critical points $C_{W}$ of a single section (20) and the expected distribution of critical points of a random section (e.g., (32) ) make it very difficult to estimate the order of magnitude of the density of critical points. A simplifying 'approximation' is to drop the absolute value around the 
determinant. The resulting density is index density for critical points. It was used in AD] and DD1] to give a lower bound for the critical point density.

To be precise, we modify (20) by defining the signed distribution of critical points of $W$ as the measure $C_{W}$ on $\mathcal{I} \backslash \widetilde{\mathcal{D}}$ given by

$$
\left\langle\operatorname{Ind}_{W}, \psi\right\rangle=\sum_{Z \in C r i t(W)}\left(\operatorname{sign} \operatorname{det} D^{2} W(Z)\right) \psi(Z, W),
$$

where $\operatorname{sign} a=1,0,-1$ if $a$ is positive, 0 , or negative, respectively. We then study the sums

$$
\mathcal{I n d}_{\psi}(L)=\sum\left\{\left\langle\operatorname{Ind}_{N}, \psi\right\rangle: N \in \mathcal{S}^{\mathbb{Z}}, Q[N] \leq L\right\} .
$$

For instance, if $\psi(Z, W)=\chi_{K}(Z)$ is the characteristic function of a compact set $K \subset \mathcal{C}$, then $\mathcal{I n d}_{\psi}(L)$ is the sum $\sum_{Z \in C r i t(W) \cap K}\left(\operatorname{sign} \operatorname{det} D^{2} W(Z)\right)$ over all non-degenerate critical points lying over $K$ of all integral flux superpotentials with $Q[W] \leq L$.

Simultaneously with Proposition 1.3, we obtain formula (1.5) of Ashok-Douglas [AD] with an estimate for the error produced by passing from the sum to the integral (cf. \&4):

TheOREM 1.8. Let $K$ be a compact subset of $\mathcal{C}$ with piecewise smooth boundary. Then

$$
\mathcal{I}_{n d_{\chi_{K}}}(L)=\frac{(\pi L)^{b_{3}}}{b_{3} ! 2^{b_{3} / 2}}\left[\int_{K} c_{m}\left(T^{*(1,0)}(\mathcal{C}) \otimes \mathcal{L}, \omega_{W P} \otimes h_{W P}^{*}\right)+O\left(L^{-1 / 2}\right)\right],
$$

where $m=\operatorname{dim} \mathcal{C}=b_{3} / 2$ and $c_{m}\left(T^{*(1,0)}(\mathcal{C}) \otimes \mathcal{L}, \omega_{W P} \otimes h_{W P}^{*}\right)=\frac{1}{\pi^{m}} \operatorname{det}\left(-R-\omega_{W P} \otimes I\right)$ is the $m$-th Chern form of $T^{*(1,0)}(\mathcal{C}) \otimes \mathcal{L}$ with respect to the Weil-Petersson metric $\omega_{W P} \otimes h_{W P}^{*}$.

Here, $R=\sum_{i j} R_{\ell i \bar{j}}^{k} d z^{i} \wedge d \bar{z}^{\bar{j}}$ is the curvature $(1,1)$ form of $T^{*(1,0)}(\mathcal{C})$ regarded as an $m \times m$ Hermitian-matrix-valued 2-form (with $m=\operatorname{dim} \mathcal{C}=b_{3} / 2$ ) and $\omega_{W P} \otimes I$ is a scalar 2-form times the $m \times m$ identity matrix. The determinant is defined as in Chern-Weil theory. The only additional step in the proof is the evaluation (given in Lemma 6.3) of the analogue of (25) in terms of the curvature form:

$$
\int_{\mathcal{S}_{Z}} \operatorname{det} H^{c} W(Z) e^{-\left\langle Q_{Z} W, W\right\rangle} d W=\left(\frac{\pi}{2}\right)^{m} \frac{\operatorname{det}\left(-R-\omega_{W P} \otimes I\right)}{d \operatorname{Vol}_{W P}} .
$$

Recall that the Chern-Gauss-Bonnet theorem tells us that if $W$ is a holomorphic section of a complex line bundle $L \rightarrow M_{m}$ over a compact complex manifold such that $\nabla W$ has only non-degenerate zeros, then

$$
c_{m}\left(T^{*(1,0)} M \otimes L\right)=\operatorname{Ind} \nabla W:=\sum_{p: \nabla W(p)=0} \operatorname{sign} \operatorname{det} H^{c} W(p) .
$$

However, the Chern-Gauss-Bonnet theorem does not apply in our setting, and indeed Ind $\nabla W$ is not constant in $W$, since $\mathcal{C}$ is an incomplete Kähler manifold and critical points can occur on the boundary or disappear. There exists a Chern-Gauss-Bonnet theorem for manifolds with boundary which expresses $\operatorname{Ind} \nabla W$ as $c_{n}(E)$ plus a boundary correction depending on $W$, but the correction term involves integrating a differential form over the boundary and that becomes problematic when the boundary is highly irregular as in the case of $\mathcal{C}$. Nevertheless, the theorem shows that asymptotically the average index density equals the Chern-Gauss-Bonnet form. 
1.6. Relations to prior results in the physics and mathematics literature. We now relate our results to the physics literature on the number of vacua and the complexity of the string theory landscape as well as to the mathematical literature on lattice points. A more detailed discussion of the landscape aspects is given in $\$ 7$

First, the string/M aspects. Over the last five years or so, many physics articles have been devoted to estimating the number of candidate vacua $N_{v a c}$ of string/M theory, in particular those which are consistent with the standard model. The candidate vacua are often pictured as valleys in a 'string theory landscape', which is the graph of the effective potential. The number of vacua is often stated as being around $10^{500}$. In [BP] Bousso-Polchinski related the number of vacua to the number of quantized fluxes $N$ satisfying a constraint $|N| \leq L$, which implies $N_{v a c}(L) \sim \frac{L^{b_{3}}}{b_{3} !}$ (see also [AD, Si]). In the specific type IIb flux compactifications studied in this paper, the constraint is hyperbolic rather than elliptic (as imagined in [BP]), and the more precise estimate $N_{v a c}(L) \sim \frac{L^{b_{3}}}{b_{3} !} f\left(b_{3}\right)$ was given in [AD, DD1] where $f\left(b_{3}\right)$ is the moduli space integral of the Gaussian integral in (32); it will be discussed further in 97. There we will also review the heuristics and the mathematics of the landscape in more detail.

What do our results imply about the number of vacua? Since Proposition 1.3 and Theorem 1.4 are asymptotic results as $L \rightarrow \infty$, they are most useful when $L^{b_{3}}$ is very large. But it is difficult to quantify 'very large' due to the complexity of the leading coefficient (24), of the remainder and of the volume of $\mathcal{C}$. Hence, we cannot make precise estimates on the number of vacua at this time.

However, to bridge our results with estimates in string theory, we make a speculative attempt in $\$ 7.3$ to draw order of magnitude conclusions from Theorem 1.4. We will use the symbol $\simeq$ in an informal sense of 'same order of magnitude' (factorial, exponential and so on). There we give a heuristic estimate of $\mathcal{K}^{\text {crit }}(Z) \simeq \frac{1}{b_{3} !}\left(b_{3} / 2\right) ! \mu^{b_{3}}$ for certain $\mu>0$. More precisely, we give heuristic upper and lower bounds with different $\mu$ which are irrelevant when comparing factorials. To obtain an order of magnitude for $\frac{f\left(b_{3}\right)}{b_{3} !}$ one would need to integrate $\mathcal{K}^{\text {crit }}$ over $\mathcal{C}$. At this time, the order of magnitude of the Weil-Petersson volume $\operatorname{Vol}_{W P}(\mathcal{C})$ of $\mathcal{C}$ is not known, even approximately $(\mathrm{Z}$. Lu). We can however make a plausible estimate for the integral of $\mathcal{K}^{\text {crit }}$ over the region where the norm of $\Lambda_{Z}$ is bounded by a uniform constant (independent of $b_{3}$ ). Since $\Lambda_{Z}$ is essentially the Hodge metric, regions where $\left\|\Lambda_{Z}\right\| \leq \mu$ are regions $K_{\mu}$ where the norm of the Ricci curvature of $\omega_{W P}$ ) is bounded above by a uniform constant. It appears likely that the volume of such regions is bounded above by the volume of balls in $\mathbb{C}^{b_{3} / 2}$ of fixed radius (Z. Lu). Since the volume of balls in $\mathbb{C}^{b_{3} / 2}$ decays like $\frac{1}{\left(b_{3} / 2\right)}$ !, we would find that the number of vacua in $K_{\mu}$ would be approximately $\frac{L^{b_{3}}}{b_{3} !} \mu^{b_{3}}$.

Now, in the physical models, $L$ is not a free parameter but is determined by $X$. In the case when there exists an involution $g$ of $X$ (an 'orientifolding') and a Calabi-Yau 4-fold $Z$ which is an elliptic fibration over $X / g$, the 'tadpole' number is then given by:

$$
\text { tadpole number : } L=\chi(Z) / 24 \text {. }
$$

In many known examples [KLRY], one has $300<b_{3}<1,000$ and $L \simeq C b_{3}$ where $1 / 3 \leq C \leq$ 3. Hence the number of vacua in $K_{\mu}$ (and possibly in all of $\mathcal{C}$ ) with the tadpole constraint $L \sim C b_{3}$ would have exponential growth $\frac{\left(C b_{3}\right)^{b_{3}}}{b_{3} !} \mu^{b_{3}}$. 
Next we turn to the purely lattice point aspects of the problem. From a mathematical point of view, this article combines statistical algebraic geometry in the sense of BSZ1, DSZ1, DSZ2 with the study of radial projections of lattice points. As far as we know, the radial projection of lattice points problem has not been studied systematically before in mathematics (we thank B. Randol for helping to sort out the historical background on this problem). The much harder problem of equidistribution of lattice points of fixed height $R$, i.e. lying on a sphere or hyperboloid of fixed radius $R$, has been studied by Yu. Linnik, C. Pommerenke $[\mathrm{Po}], \mathrm{W}$. Duke and others. But the remainders obtained in this more delicate problem are not as accurate as ours are for the bulk problem of projecting all lattice points of height $<R$. Counting projections of lattice points in domains of a hypersurface is equivalent to counting lattice points in certain cones, and there are some additional studies of this by methods of automorphic forms. In certain right circular cones with a flat top, Duke and Imamoglu [DO] use Dirichlet series and Shimura lifts to obtain the leading order asymptotics. Radial projections of lattice points additionally bear some resemblance to rational points. Some results and references for this problem are contained in [DO]. In [Ze2], the general problem of counting radial projections of lattice points in smooth domains of non-degenerate hypersurfaces is studied. In [NR], some further results are given on radial projections of lattice points, in particular in the case of hypersurfaces with flat spots or in the case of polyhedra.

Acknowledgement: We would like to thank Zhiqin Lu for many helpful comments regarding the Weil-Petersson and Hodge metrics on the moduli space of a Calabi-Yau 3-fold. In particular, our discussion of the Weil-Petersson volume $V_{W P}(\mathcal{C})$ and estimates of the eigenvalues of $\Lambda_{Z}$ are based on his remarks.

\section{Background on CAlabi-Yau manifolds AND COMPlex GeOMETRY}

As mentioned in the introduction, the supersymmetric vacua of type IIb flux compactifications on a $C Y_{3}$ are critical points of holomorphic sections of the holomorphic line bundle $\mathcal{L} \rightarrow \mathcal{C}$ dual to the Hodge bundle $H^{4,0}\left(X \times T^{2}\right)$, where the configuration space $\mathcal{C}$ is the moduli space $\mathcal{M} \times \mathcal{E}$ of product complex structures on $X \times T^{2}$. In this section, we give the geometric background necessary for the analysis of critical points and Hessians of the holomorphic sections $W_{G}$ of (5) .

The most significant aspects of Calabi-Yau geometry in the study of critical points of flux superpotentials are the following:

- The space $\mathcal{S}_{Z}$ of flux superpotentials with $\nabla W_{G}(Z)=0$ may be identified with the space $H_{Z}^{3}(X)$ of fluxes $G=F+i H$ with the special Hodge decomposition $F+\tau H \in$ $H_{z}^{2,1}(X) \oplus H_{z}^{0,3}(X)$. See Proposition 3.1 .

- The space $H_{z}^{2,1}(X) \oplus H_{z}^{0,3}(X) \subset H^{3}(X, \mathbb{C})$ is a positive complex Lagrangian subspace. See Proposition 2.1. Hence, $\mathcal{S}_{Z}$ is endowed with an inner product.

In addition, we review the relation between holomorphic derivatives, covariant derivatives and second fundamental forms for holomorphic frames $\Omega_{z}$ of the Hodge bundle, and recall the definition of the prepotential. These results are needed for the calculations in Lemmas 107] and 6.1. Much of this material is essentially standard [CO, St1, DD1, but it is not always stated precisely in the physics sources. We therefore present it here for the sake of clarity and completeness. 
2.1. Geometry of Calabi-Yau manifolds. We recall that a Calabi-Yau $d$-fold $M$ is a complex $d$-dimensional manifold with trivial canonical bundle $K_{M}$, i.e. $c_{1}(M)=0$. By the well-known theorem of Yau, there exists a unique Ricci flat Kähler metric in each Kähler class on $M$. In this article, we fix the Kähler class, and then the Calabi-Yau metrics correspond to the complex structures on $M$ modulo diffeomorphisms. We denote the moduli space of complex structures on $M$ by $\mathcal{M}$.

As mentioned in the introduction, the Calabi-Yau manifolds of concern in this article are the 4-folds $M=X \times T^{2}$, where $T^{2}=\mathbb{R}^{2} / \mathbb{Z}^{2}$. The $T^{2}$ factor plays a special role, and the geometric aspects mainly concern $X$. We only consider product Calabi-Yau metrics and complex structures on $M$. Thus, the configuration space $\mathcal{C}=\mathcal{M} \times \mathcal{E}$ where $\mathcal{M}$ is the moduli space of complex structures on $X$ and where $\mathcal{E}$ is the moduli space of elliptic curves. We denote a point of $\mathcal{C}$ by $Z=(z, \tau)$ where $z$ denotes a complex structure on $X$ and where $\tau$ denotes the complex structure on $T^{2}$ corresponding to the elliptic curve $\mathbb{C} / \mathbb{Z} \oplus \mathbb{Z} \tau$.

It is often simplest to view the moduli space of complex structures on $X$ as the quotient by the mapping class group $\Gamma$ of the Teichmüller space $\mathcal{T}$ eich $(X)$, where

$$
\text { Teich }(X)=\{\text { complex structures on } X\} / \text { Diff }_{0}
$$

where $J \sim J^{\prime}$ if there exists a diffeomorphism $\varphi \in$ Diff $_{0}$ isotopic to the identity satisfying $\varphi^{*} J^{\prime}=J$. The mapping class group is the group of connected components of the diffeomorphism group,

$$
\Gamma_{X}:=\operatorname{Diff}(X) / \operatorname{Diff}_{0}(X) .
$$

We shall identify $\mathcal{M}$ with a fundamental domain for $\Gamma_{X}$ in $\mathcal{T}$ eich $(X)$, and $\mathcal{E}$ with the usual modular domain in $\mathcal{H}$.

The mapping class group for a Calabi-Yau $d$-fold has a representation on $H^{d}(M, \mathbb{R})$ which preserves the intersection form $Q$, which is symplectic in odd dimensions, and indefinite symmetric in even dimensions. In odd dimensions, this representation gives a homomorphism $\varphi: \Gamma_{M} \rightarrow \operatorname{Sp}\left(b_{d}(M), \mathbb{Z}\right)$, while in even dimensions it gives a homomorphism to the corresponding orthogonal group. It was proved by D. Sullivan Sul that if $d \geq 3$, then $\varphi\left(\Gamma_{M}\right)$ is an (arithmetic) subgroup of finite index (in $\operatorname{Sp}\left(b_{d}(M), \mathbb{Z}\right)$ if $d$ is odd), and the kernel of $\varphi$ is a finite subgroup.

On any CY manifold $M$ of dimension $d$, the space $H_{z}^{d, 0}(M)$ of holomorphic $(d, 0)$ forms for a complex structure $Z$ is one-dimensional. It depends holomorphically on $Z$ and hence defines a complex holomorphic line bundle $\mathcal{L}_{\mathcal{M}}^{*}=H^{d, 0} \rightarrow \mathcal{M}$, which we refer to as the 'Hodge bundle.' The Hodge bundle is equipped with the Weil-Petersson (WP) Hermitian metric of (10), which we repeat here:

$$
h_{W P}\left(\Omega_{z}, \Omega_{z}\right)=i^{d^{2}} \int_{X} \Omega \wedge \bar{\Omega}
$$

For a holomorphic Hermitian line bundle $(L, h) \rightarrow M$ and local holomorphic frame $e_{L}$ over an open set $U \subset M$, we write

$$
\left|e_{L}(z)\right|_{h}^{2}=e^{-K(z)} .
$$

The connection 1-form in this frame is the $(1,0)$ form $-\partial K(z)$, and the curvature $(1,1)$-form is given by

$$
\omega=\frac{i}{2} \Theta_{h}=\frac{i}{2} \partial \bar{\partial} K, \quad K=-\log \left|e_{L}\right|_{h}^{2}
$$


The Hermitian line bundle is said to be positive if $\omega$ is a positive $(1,1)$ form, in which case $K$ is called the Kähler potential. The Hermitian line bundle $(L, h)$ is negative if $\omega$ is a negative $(1,1)$ form.

In particular, the curvature of the Weil-Petersson metric on $H^{d, 0} \rightarrow \mathcal{M}$ is a positive $(1,1)$ form on $\mathcal{M}$, and hence it defines a Kähler form with potential (with respect to the frame $\left.\Omega_{z}\right)$

$$
K_{W P}=-\log h_{W P}\left(\Omega_{z}, \Omega_{z}\right)=-\log i \int_{X} \Omega \wedge \bar{\Omega} .
$$

For instance, consider the Hodge bundle $H_{\tau}^{1,0} \rightarrow \mathcal{E}$. It has a standard frame $d x+\tau d y$ for which $K=-\log \operatorname{Im} \tau$. Here, $\tau$ is the standard coordinate on the upper half plane. Then $\partial K=-\frac{1}{\tau-\bar{\tau}} d \tau$ and the Kähler form is $-\frac{i}{2(\tau-\bar{\tau})^{2}} d \tau \wedge d \bar{\tau}>>0$.

In the product situation of $M=X \times T^{2}, H_{z, \tau}^{4,0}\left(X \times T^{2}\right)=H_{z}^{3,0}(X) \otimes H_{\tau}^{1,0}\left(T^{2}\right)$. Thus, the line bundle $H^{4,0}\left(X \times T^{2}\right) \simeq H^{3,0}(X) \otimes H^{1,0}\left(T^{2}\right) \rightarrow \mathcal{C}$ is an exterior tensor product and the WP metric is a direct product. We denote an element of $H_{z}^{3,0}(X)$ by $\Omega_{z}$, and an element of $H_{\tau}^{1,0}\left(T^{2}\right)$ by $\omega_{\tau}$. We often assume that $\omega_{\tau}=d x+\tau d y$.

2.2. Variational derivatives and covariant derivatives. The bundle $H_{z}^{3,0}(X) \rightarrow \mathcal{M}$ is a holomorphic line bundle. Since $H_{z}^{3,0}(X) \subset H^{3}(X, \mathbb{C})$, one can view a holomorphically varying family $\Omega_{z} \in H_{z}^{3,0}(X)$ as a holomorphic map $\mathcal{M} \rightarrow H^{3}(X, \mathbb{C})$ or as a holomorphic section of $H_{z}^{3,0}(X)$. As a holomorphic vector valued function, $\Omega_{z}$ may be differentiated in $z$. If $z_{1}, \ldots, z_{h^{2,1}}$ are local holomorphic coordinates, and if $\left\{\frac{\partial}{\partial z_{j}}\right\}$ are the coordinate vector fields, then $\frac{\partial \Omega}{\partial z_{j}}$ is a well-defined element of $H^{3}(X, \mathbb{C})$.

By the Griffiths transversality theorem, see [GHJ], CO], (5.4)) or [Wa1, Wa2],

$$
\frac{\partial \Omega_{z}}{\partial z^{j}}=k_{j}(z) \Omega_{z}+\chi_{j}
$$

where $\chi_{j} \in H_{z}^{2,1}(X)$ and where $k \in C^{\infty}(\mathcal{M})$. Note that although $\frac{\partial \Omega_{z}}{\partial z^{j}}$ is holomorphic, neither term on the right hand side is separately holomorphic.

We define a Levi-Civita connection on the bundle $H^{3,0} \rightarrow \mathcal{M}$ by orthogonally projecting the derivatives $\frac{\partial \Omega_{z}}{\partial z^{j}}$ onto $H^{3,0}$. This defines the Weil-Petersson connection $\nabla_{W P}$ on $H^{3,0} \rightarrow$ $\mathcal{M}$

$$
\nabla_{W P}: C^{\infty}(\mathcal{M}, \mathcal{L}) \rightarrow C^{\infty}\left(\mathcal{M}, \mathcal{L} \otimes T^{*}\right)
$$

It follows from (40) that

$$
\frac{\partial}{\partial z^{j}} \int_{X} \Omega_{z} \wedge \bar{\Omega}_{z}=k_{j} \int_{X} \Omega_{z} \wedge \bar{\Omega}_{z}
$$

which by (39) implies

$$
k_{j}=-\frac{\partial K}{\partial z^{j}} .
$$

Hence,

$$
\nabla_{W P} \Omega_{z}=-\partial K \otimes \Omega_{z}=\sum k_{j} d z_{j} \otimes \Omega_{z}
$$

is the Chern connection of the Weil-Petersson Hermitian metric. 
We also define the forms

$$
\left\{\begin{array}{l}
\mathcal{D}_{j} \Omega_{z}=\frac{\partial}{\partial z^{j}} \Omega+\frac{\partial K}{\partial z^{j}} \Omega \\
\mathcal{D}_{j} \mathcal{D}_{k} \Omega_{z}=\left(\frac{\partial}{\partial z^{j}}+\frac{\partial K}{\partial z^{j}}\right)\left(\frac{\partial}{\partial z^{k}}+\frac{\partial K}{\partial z^{k}}\right) \Omega_{z} .
\end{array}\right.
$$

We then have

$$
\mathcal{D}_{j} \Omega_{z}=\frac{\partial \Omega_{z}}{\partial z^{j}}-k_{j} \Omega_{z}=\chi_{j} \in H^{2,1}\left(X_{z}\right)
$$

The operator $\mathcal{D}_{j} \Omega_{z}$ is analogous to the second fundamental form $I I(X, Y)=\left(\tilde{\nabla}_{X} Y\right)^{\perp}$ of an embedding, i.e. it is the 'normal' component of the ambient derivative. It is known that the first variational derivatives span $H^{2,1}$ (see e.g. Wa1, Wa2. (In the physics literature, $D_{\alpha}$ is often described as a connection, and is often identified with $\nabla_{W P}$, but this is not quite correct as it is applied to $\Omega_{z}$ ).

The Weil-Petersson Hermitian metric $\sum G_{i j} d z_{i} d \bar{z}_{\bar{j}}$ on $\mathcal{M}$ is the curvature $(1,1)$-form of the Hodge bundle. From (39) and (44), we have:

$$
G_{j \bar{k}}=\frac{\partial^{2}}{\partial z^{j} \partial \bar{z}^{k}} K(z, \bar{z})=-\frac{\int_{\mathcal{M}} \mathcal{D}_{j} \Omega_{z} \wedge \overline{\mathcal{D}_{k} \Omega_{z}}}{\int_{\mathcal{M}} \Omega_{z} \wedge \bar{\Omega}_{z}} .
$$

2.3. Yukawa couplings and special geometry of the moduli space. In formula (32), the density of critical points is expressed as an integral over a space $\mathcal{H}_{Z} \oplus \mathbb{C}$, where $\mathcal{H}_{Z}$ is a subspace of the complex symmetric matrices $\operatorname{Sym}\left(h^{2,1}+1, \mathbb{C}\right)$ spanned by the special matrices $\xi^{j}$ given in (27). Their components $\mathcal{F}_{i k}^{\bar{j}}(z)$ are known as Yukawa couplings and defined as follows: A priori, $\mathcal{D}_{k} \mathcal{D}_{j} \Omega_{z} \in H^{2,1} \oplus H^{1,2}$, and moreover its $H^{2,1}$ component vanishes (see e.g. CO, (5.5)]). Hence we may define $\mathcal{F}_{k j}^{\bar{l}}$ by

$$
\mathcal{D}_{k} \mathcal{D}_{j} \Omega_{z}=-\sqrt{-1} e^{K} \mathcal{F}_{k j}^{\bar{l}} \overline{\mathcal{D}_{l} \Omega} \quad\left(1 \leq j, k, l \leq h^{2,1}\right) .
$$

See also [St1, (28)]. It is further shown in [St1, (37)] (see also [AD, (4.8)], [LS2, Theorem 3.1]) that the Riemann tensor of the Weil-Petersson metric on the moduli space $\mathcal{M}$ of Calabi-Yau three-folds is related to the Yukawa couplings by

$$
R_{i \bar{j} k \bar{\ell}}=G_{i \bar{j}} G_{k \bar{\ell}}+G_{i \bar{\ell}} G_{k \bar{j}}-e^{2 K} \sum_{p, q} G^{p \bar{q}} \mathcal{F}_{i k p} \overline{\mathcal{F}_{j \ell q}} .
$$

The Yukawa couplings are related to the periods of $\Omega_{z}$ and to the so-called prepotential of $\mathcal{M}$. We pause to recall the basic relations and to direct the reader to the relevant references.

First, we consider periods. As a basis of $H_{3}(X, \mathbb{R})$ we choose the symplectic basis consisting of dually paired Lagrangian subspaces of $A$-cycles $A_{a}$ and $B$-cycles $B_{a}$. The periods of $\Omega_{z} \in H_{z}^{3,0}(X)$ over the $A$-cycles

$$
\zeta^{a}=\int_{A_{a}} \Omega_{z} \quad\left(1 \leq a \leq h^{2,1}+1=b_{3} / 2\right)
$$

define holomorphic coordinates on $\mathcal{L}_{\mathcal{M}}^{*}=H^{3,0} \rightarrow \mathcal{M}$. Alternately, we can view the $\zeta^{a}$ as 'special' projective coordinates on $\mathcal{M}$. The periods of $\Omega_{z}$ over the $B$-cycles are then holomorphic functions of the $\zeta^{a}$. The principal fact is that the image of $\mathcal{L}_{\mathcal{M}}^{*}$ under the 
period map is a complex Lagrangian submanifold of $H^{3}(M, \mathbb{C})$, and thus is determined by a single holomorphic function, the "prepotential" $\mathcal{F}=\mathcal{F}\left(\zeta^{1}, \ldots, \zeta^{b_{3} / 2}\right): \mathcal{L}_{\mathcal{M}}^{*} \rightarrow \mathbb{C}$ such that

$$
\int_{B_{a}} \Omega_{z}=\frac{\partial \mathcal{F}}{\partial \zeta^{a}}
$$

Furthermore, $\mathcal{F}$ is homogeneous of degree 2 in the periods $\zeta^{a}$,

$$
\sum_{j=1}^{b_{3} / 2} \zeta^{a} \frac{\partial \mathcal{F}}{\partial \zeta^{a}}=2 \mathcal{F}(z),
$$

and hence may be viewed as a holomorphic section of $\mathcal{L}_{\mathcal{M}}^{\otimes 2}$.

The local holomorphic 3-form $\Omega_{z}$ may be expressed in terms of the Poincaré duals of the symplectic basis by:

$$
\Omega_{z}=\sum_{a=1}^{b_{3} / 2}\left(\zeta^{a} \widehat{A}_{a}-\frac{\partial \mathcal{F}}{\partial \zeta^{a}} \widehat{B}_{a}\right) .
$$

(See CO, (3.8)). Further, in these coordinates, the Kähler potential (39) of the WeilPetersson metric may be written as

$$
K(z, \bar{z})=-\log i\left(\sum_{a=1}^{b_{3} / 2} \zeta^{a} \frac{\overline{\partial \mathcal{F}}}{\partial \zeta^{a}}-\overline{\zeta^{a}} \frac{\partial \mathcal{F}}{\partial \zeta^{a}}\right) .
$$

We also have:

$$
\mathcal{F}_{k j}^{\bar{l}}=\sum_{r=1}^{h^{2,1}} G^{r \bar{l}} \frac{\partial^{3} \mathcal{F}}{\partial z^{r} \partial z^{j} \partial z^{k}}
$$

See [CO, (4.5)] and [St1, (64)].

In summary, we reproduce the table from [CO]:

$$
\begin{array}{lc}
\text { Derivatives of the Basis } & \text { spans } \\
\Omega & H^{3,0} \\
\mathcal{D}_{j} \Omega & H^{2,1} \\
\mathcal{D}_{k} \mathcal{D}_{j} \Omega=-i e^{K} \mathcal{F}_{k j}^{\bar{\gamma}} \overline{\mathcal{D}_{\gamma} \Omega} & H^{12} \\
\mathcal{D}_{k} \mathcal{D}_{\bar{j}} \Omega=G_{k \bar{j}} \bar{\Omega} & H^{03}
\end{array}
$$

2.3.1. $\mathcal{C}$ as the moduli space of complex structures on $X \times T^{2}$. Above, we have reviewed the geometry of the moduli space of complex structures on the Calabi-Yau three-fold. Our configuration space $\mathcal{C}=\mathcal{M} \times \mathcal{H}$ may be viewed as (a component of) the moduli space of complex structures on $X \times T^{2}$. This point of view is used in DD1, but because the $T^{2}$ factor plays a distinguished role we do not emphasize this identification here. Further, formula (47) needs to be modified for the moduli space of complex structures on a Calabi-Yau four-fold. 
In [LS2, Theorem 3.1]), the Riemann tensor of the Weil-Petersson metric on the moduli space of a Calabi-Yau manifold of arbitrary dimension is shown to be

$$
R_{i \bar{j} k \bar{\ell}}=G_{i \bar{j}} G_{k \bar{\ell}}+G_{i \bar{\ell}} G_{k \bar{j}}-\frac{\left\langle\mathcal{D}_{k} \mathcal{D}_{i} \Omega, \overline{\left.\mathcal{D}_{\ell} \mathcal{D}_{j} \Omega\right\rangle}\right.}{\int_{\mathcal{M}} \Omega \wedge \bar{\Omega}} .
$$

In the case of three-folds, the vectors $\mathcal{D}_{j} \Omega$ form an orthonormal basis for $H^{2,1}$ and one can write the inner product in the form (47).

2.4. Hodge-Riemann form and inner products. The Hodge-Riemann bilinear form on $H^{3}(X, \mathbb{R})$ is the intersection form $(\alpha, \beta) \mapsto \int_{X} \alpha \wedge \beta$. We consider the sesquilinear pairing:

$$
(\alpha, \beta) \mapsto Q(\alpha, \bar{\beta})=-\sqrt{-1} \int_{X} \alpha \wedge \bar{\beta}, \quad \alpha, \beta \in H^{3}(X, \mathbb{C})
$$

An important fact is that under the Hodge decomposition (3) for a given complex structure, the Hodge-Riemann form is definite in each summand:

$$
(-1)^{p} Q(\alpha, \bar{\alpha})>0, \quad \alpha \in H^{p, 3-p}(X, \mathbb{C}),
$$

whose sign depends only on the parity of $p$. (See [GH, $\S 7$. Note that our definition of $Q$ has the extra sign $-\sqrt{-1}$. The inequality (54) holds only for primitive forms, but in our case all harmonic 3-forms are primitive, since we are assuming that $H^{1}(M, \mathbb{C})=0$.) To restate (54):

Proposition 2.1. Let $\operatorname{dim} X=3$, and let $b_{1}(X)=0$. Then for each $z \in \mathcal{M}$, the HodgeRiemann form is positive definite on $H_{z}^{2,1} \oplus H_{z}^{0,3}$ and negative definite on $H_{z}^{3,0} \oplus H_{z}^{1,2}$.

By Griffiths transversality (see (40)), for any local holomorphic frame $\Omega_{z}, \mathcal{D}_{j} \Omega_{z} \in H_{z}^{2,1}$ and these elements span $H_{z}^{2,1}$. Also, $\bar{\Omega}_{z}$ spans $H^{0,3}$. These forms provide us with an orthonormal basis for $H_{z}^{2,1} \oplus H_{z}^{0,3}$ :

Proposition 2.2. If $\left\{z_{j}\right\}$ are coordinates at $z_{0}$ such that $\left\{\partial /\left.\partial z_{j}\right|_{z_{0}}\right\}$ are orthonormal, and if $h_{W P}\left(\Omega_{z_{0}}, \Omega_{z_{0}}\right)=1$, then the basis $\left\{\mathcal{D}_{j} \Omega_{z_{0}}, \bar{\Omega}\left(z_{0}\right)\right\}$ is a complex orthonormal basis of $H_{z_{0}}^{2,1} \oplus$ $H_{z_{0}}^{0,3}$ with respect to the Hodge Riemann form $Q$.

Remark: Here and below, when we say that a basis of a complex vector space is complex orthonormal we mean that it is a complex basis and is orthonormal for the given inner product. By a real orthonormal basis of the same vector space we mean an orthonormal basis of the underlying real vector space.

Proof. It suffices to show that:

$$
\begin{aligned}
& \text { (i) } Q\left(\mathcal{D}_{j} \Omega_{z}, \overline{\mathcal{D}_{k} \Omega_{z}}\right)=-i \int_{X} \mathcal{D}_{j} \Omega_{z} \wedge \overline{\mathcal{D}_{k} \Omega_{z}}=G_{j \bar{k}} e^{-K} \\
& \text { (ii) } Q\left(\mathcal{D}_{j} \Omega_{z}, \Omega_{z}\right)=-i \int_{X} \mathcal{D}_{\bar{j}} \Omega_{z} \wedge \Omega_{z}=0 \\
& \text { (iii) } Q\left(\bar{\Omega}_{z}, \Omega_{z}\right)=-i \int_{X} \bar{\Omega}_{z} \wedge \Omega_{z}=h_{W P}\left(\Omega_{z}, \Omega_{z}\right)
\end{aligned}
$$

Equation (i) follows from (45), (ii) is by type considerations, and (iii) follows from (10). 
Remark: In the language of complex symplectic geometry, Proposition 2.1 says that $H_{z}^{2,1} \oplus$ $H_{z}^{0,3}$ is a positive complex polarization of $H^{3}(X, \mathbb{C})$. Let us recall the definitions. The space $\left(H^{3}(X, \mathbb{R}), Q\right)$ of real 3-cycles with its intersection form $Q(\alpha, \beta)=-i \int_{M} \alpha \wedge \beta$ is a real symplectic vector space. After complexifying, we obtain the complex symplectic vector space $\left(H^{3}(X, \mathbb{C}), Q\right)$. In general, if $(V, \omega)$ is a real symplectic vector space and if $\left(V_{\mathbb{C}}, \omega_{\mathbb{C}}\right)$ is its complexification, a complex Lagrangian subspace $F \subset V_{\mathbb{C}}$ is called a polarization. The polarization is called real if $F=\bar{F}$ and complex if $F \cap \bar{F}=\{0\}$. The polarization $F$ is called positive if $i \omega(v, \bar{w})$ is positive definite on $F$.

In our setting, $(V, \omega)=\left(H^{3}(X, \mathbb{R}), Q\right)$. We observe that for any complex structure $z$ on $X$ (as a complex manifold), the Hodge decomposition may be written in the form

$$
H^{3}(X, \mathbb{C})=F \oplus \bar{F}, \quad F=H^{2,1} \oplus H^{0,3} \bar{F}=H^{3,0} \oplus H^{1,2}, \quad,
$$

where $F$ is complex Lagrangian. By Proposition 2.1 this polarization is positive, i.e.

$$
Q(v, \bar{v})>0, \quad v \in F \backslash\{0\} .
$$

\section{CRitical points of Superpotentials}

In this section, we assemble some basic facts about critical points and Hessians of flux superpotentials.

3.1. Flux superpotentials as holomorphic sections. As discussed in the previous section, $\mathcal{L} \rightarrow \mathcal{C}$ is a negative line bundle. On a compact complex manifold, a negative line bundle has no holomorphic sections. However, $\left(\mathcal{C}, \omega_{W P}\right)$ is a non-compact, incomplete Kähler manifold of finite Weil-Petersson volume (see [LS1] for the latter statement), and the line bundle $\mathcal{L} \rightarrow \mathcal{C}$ has many holomorphic sections related to the periods of $X \times T^{2}$.

The sections relevant to this article are the flux superpotentials $W_{G}$ of (15)-(6). $W_{G}$ depends on two real fluxes $F, H \in H^{3}(X, \mathbb{Z})$, which we combine into a complex integral flux

$$
G=F+i H \in H^{3}(X, \mathbb{Z} \oplus \sqrt{-1} \mathbb{Z}) .
$$

The main reason to form this complex combination is that it relates the tadpole constraint (11) on the pair $(F, H)$ to the Hodge-Riemann form (2). However, none of subsequent identifications preserves this complex structure, and the reader may prefer to view $G$ as just the pair $G=(F, H) \in H^{3}(X, \mathbb{Z}) \oplus H^{3}(X, \mathbb{Z})$. Alternately, we can identifying $G=F+i H \in H^{3}(X, \mathbb{C})$ with the real cohomology class

$$
\widetilde{G}:=F \wedge d y-H \wedge d x \in H^{4}\left(X \times T^{2}, \mathbb{R}\right) \approx H^{3}(X, \mathbb{C}) .
$$

We shall consider the (real-linear) embedding

$$
\mathcal{W}: H^{3}(X, \mathbb{C}) \rightarrow H^{0}(\mathcal{C}, \mathcal{L}), \quad G \mapsto W_{G},
$$

where $W_{G}$ is given by formula (6) ; i.e.,

$$
\left(W_{G}(z, \tau), \Omega_{z} \otimes \omega_{\tau}\right)=\int_{X \times T^{2}} \widetilde{G} \wedge \Omega_{z} \wedge \omega_{\tau} .
$$

We denote by $\mathcal{S}=\operatorname{Image}(\mathcal{W})$ the range of this map, and by

$$
\mathcal{S}^{\mathbb{Z}}=\mathcal{W}\left(H^{3}(X, \mathbb{Z} \oplus i \mathbb{Z})\right)
$$


the lattice of sections satisfying the integrality condition. The map $G \rightarrow W_{G}$ is not complex linear, so $\mathcal{S}$ is not a complex subspace of $H^{0}(\mathcal{M} \times \mathcal{E}, \mathcal{L})$. Rather, it is a real subspace of dimension $2 b_{3}$ (over $\mathbb{R}$ ) and $\mathcal{S}^{\mathbb{Z}}$ is a lattice of rank $2 b_{3}$ in it. In fact $\mathcal{S} \approx \mathbb{R}^{2 b_{3}}$ is totally real in $H^{0}(\mathcal{C}, \mathcal{L}) \approx \mathbb{C}^{2 b_{3}}$.

We choose local holomorphic frames $\Omega_{z}$ of the Hodge bundle $H^{3,0} \rightarrow \mathcal{M}$ and $\omega_{\tau}=d x+\tau d y$ of $H^{1,0} \rightarrow \mathcal{E}$ and let $\Omega_{z}^{*} \otimes \omega_{\tau}^{*}$ denote the dual co-frame of $\mathcal{L}$. A holomorphic section of $\mathcal{L}$ can then be expressed as $W=f(z, \tau) \Omega_{z}^{*} \otimes \omega_{\tau}^{*}$ where $f \in \mathcal{O}(\mathcal{C})$ is a local holomorphic function. If $W=W_{G}$ is a flux superpotential, then the corresponding function $f_{G}$ is given by:

$$
f_{G}(z, \tau)=\int_{X \times T^{2}}(F \wedge d y-H \wedge d x) \wedge\left(\Omega_{z} \wedge \omega_{\tau}\right)
$$

When $\omega_{\tau}=d x+\tau d y$ (on a fundamental domain in Teichmüller space), we obtain the simpler form:

$$
f_{G}(z, \tau)=\int_{X}(F+\tau H) \wedge \Omega_{z}
$$

3.2. Critical points and Hessians of holomorphic sections. As preparation for critical points of superpotentials, we recall some basic notations and facts concerning critical points and Hessians of holomorphic sections of a general line bundle $L \rightarrow M$ (see [DSZ1]).

Let $(L, h) \rightarrow M$ be a holomorphic Hermitian line bundle, let $e_{L}$ denote a local frame over an open set $U$ and write a general holomorphic section as $s=f e_{L}$ with $f \in \mathcal{O}(U)$. Recall that the Chern connection $\nabla_{h}$ of $h$ is given locally as $\nabla\left(f e_{L}\right)=(\partial f-f \partial K) \otimes e_{L}$, where $K=-\log \left\|e_{L}\right\|_{h}^{2}$, i.e.

$$
\nabla s=\sum_{j=1}^{m}\left(\frac{\partial f}{\partial z^{j}}-f \frac{\partial K}{\partial z^{j}}\right) d z^{j} \otimes e_{L}=\sum_{j=1}^{m} e^{K} \frac{\partial}{\partial z^{j}}\left(e^{-K} f\right) d z^{j} \otimes e_{L} .
$$

The critical point equation thus reads,

$$
\frac{\partial f}{\partial z^{j}}-f \frac{\partial K}{\partial z^{j}}=0
$$

The Hessian of a holomorphic section $s$ of $(L, h) \rightarrow M$ at a critical point $Z_{0}$ is the tensor

$$
D \nabla s\left(Z_{0}\right) \in T^{*} \otimes T^{*} \otimes L
$$

where $D$ is a connection on $T^{*} \otimes L$. At a critical point $Z_{0}, D \nabla s\left(Z_{0}\right)$ is independent of the choice of connection on $T^{*}$. In a local frame and in local coordinates we have

$$
D^{\prime} \nabla^{\prime} s\left(Z_{0}\right)=\sum_{j, q} H_{j q}^{\prime} d z^{q} \otimes d z^{j} \otimes e_{L}, \quad D^{\prime \prime} \nabla^{\prime} s\left(Z_{0}\right)=\sum_{j, q} H_{j q}^{\prime \prime} d \bar{z}^{q} \otimes d z^{j} \otimes e_{L} .
$$

The Hessian $D \nabla s\left(Z_{0}\right)$ at a critical point thus determines the complex symmetric matrix $H^{c}$ (which we call the 'complex Hessian'):

$$
H^{c}:=\left(\begin{array}{ll}
H^{\prime} & H^{\prime \prime} \\
\overline{H^{\prime \prime}} & \overline{H^{\prime}}
\end{array}\right)=\left(\begin{array}{cc}
H^{\prime} & -f\left(Z_{0}\right) \Theta \\
-\overline{f\left(Z_{0}\right) \Theta} & \overline{H^{\prime}}
\end{array}\right)
$$


whose components are given by

$$
\begin{aligned}
& H_{j q}^{\prime}=\left(\frac{\partial}{\partial z^{j}}-\frac{\partial K}{\partial z^{j}}\right)\left(\frac{\partial}{\partial z^{q}}-\frac{\partial K}{\partial z^{q}}\right) f\left(Z_{0}\right) \\
& H_{j q}^{\prime \prime}=-\left.f \frac{\partial^{2} K}{\partial z^{j} \partial \bar{z}^{q}}\right|_{Z_{0}}=-f\left(Z_{0}\right) \Theta_{j q}, \quad \Theta_{h}\left(Z_{0}\right)=\sum_{j, q} \Theta_{j q} d z^{j} \wedge d \bar{z}^{q} .
\end{aligned}
$$

3.3. Supersymmetric critical points and the Hodge decomposition. We now specialize to the critical point equations for flux superpotentials $W_{G}(z, \tau)$. An important observation that is now standard in the physics literature is that the complex moduli $(z, \tau)$ at which a flux superpotential $W_{G}(z, \tau)$ satisfies $\nabla W_{G}=0$ are characterized by the following special Hodge decomposition of $H^{3}(X, \mathbb{C}$ ) at $z$ (see AD, $(3.5)-(3.8)$ ).

A local holomorphic frame for the Hodge bundle $\mathcal{L} \rightarrow \mathcal{C}$ is $e_{\mathcal{L}}=\Omega_{z}^{*} \otimes \omega_{\tau}^{*}$, where $\Omega_{z}^{*}$ is dual to the local frame $\Omega_{z}$ of the Hodge line bundle $H^{3,0} \rightarrow \mathcal{M}$ and $\omega_{\tau}^{*}$ is dual to the local frame $\omega_{\tau}=d x+\tau d y$ of $H^{1,0} \rightarrow \mathcal{E}$. We let $K(Z)=K_{X}(z)+K_{T^{2}}(\tau)$ be the Kähler potential for the local frame $\Omega_{z} \otimes \omega_{\tau}$ of the (positive) Hodge bundle $\mathcal{L}^{*}$. We then have

$$
\left|e_{\mathcal{L}}(Z)\right|_{h}^{2}=\left|\Omega_{z} \otimes \omega_{\tau}\right|_{h_{W P}}^{-2}=e^{K(Z)}=e^{K_{X}(z)} e^{K_{T^{2}}(\tau)} .
$$

Hence, the Weil-Petersson Kähler potential on $\mathcal{C}$ is

$$
K(Z)=-\log \int_{X} \Omega_{z} \wedge \bar{\Omega}_{z}-\log (\bar{\tau}-\tau) .
$$

In particular, the $\tau$-covariant derivative on $\mathcal{L}$ is given in the local frame $e_{\mathcal{L}}$ by

$$
\nabla_{\tau}=\frac{\partial}{\partial \tau}+\frac{1}{\bar{\tau}-\tau}
$$

Hence with $W_{G}=f_{G} e_{\mathcal{L}}$, we have

$$
\begin{aligned}
\nabla_{\tau} f_{G} & =\int_{X}\left[H+\frac{1}{\bar{\tau}-\tau}(F+\tau H)\right] \wedge \Omega_{z} \\
& =\frac{1}{\bar{\tau}-\tau} \int_{X}(F+\bar{\tau} H) \wedge \Omega_{z} .
\end{aligned}
$$

To compute the $z$-derivatives, we see from $\$ 2.2$ and (56) -(57) that

$$
\begin{aligned}
\nabla_{z^{j}} f_{G} & =\left(\frac{\partial f_{G}}{\partial z^{j}}+\frac{\partial K}{\partial z^{j}} f_{G}\right)(z, \tau)=\int_{X}(F+\tau H) \wedge\left(\frac{\partial \Omega_{z}}{\partial z^{j}}+\frac{\partial K}{\partial z^{j}} \Omega_{z}\right) \\
& =\int_{X}(F+\tau H) \wedge \chi_{j}=0,
\end{aligned}
$$

for $1 \leq j \leq h^{2,1}$. Thus, the supersymmetric critical point equations for the flux superpotential $W_{G}$ read:

$$
\left\{\begin{array}{l}
\int_{X}(F+\tau H) \wedge \mathcal{D}_{j} \Omega_{z}=0 \quad\left(1 \leq j \leq h^{2,1}\right) \\
\int_{X}(F+\tau H) \wedge \bar{\Omega}_{z}=0 .
\end{array}\right.
$$

As in (16), we denote by $\mathcal{S}_{Z}(Z=(\tau, z))$ the space of superpotentials $W_{G}$ with $\nabla W_{G}(Z)=$ 0 . Although the equation is complex linear on $H^{0}(\mathcal{C}, \mathcal{L}), \mathcal{S}$ is not a complex subspace of 
$H^{0}(\mathcal{C}, \mathcal{L})$, so $\mathcal{S}_{Z}$ is a real but not complex vector space. Put another way, for each $Z=(z, \tau)$, the critical point equation determines a real subspace

$$
H_{Z}^{3}(X, \mathbb{C})=\mathcal{W}^{-1}\left(\mathcal{S}_{Z}\right)=\left\{F+i H, \quad F, H \in H^{3}(X, \mathbb{R}), \text { (66) is true }\right\} .
$$

The critical point equations (66) put $b_{3}=2\left(h^{2,1}+1\right)$ independent real linear conditions on $2 b_{3}$ real unknowns $(F, H)$.

Proposition 3.1. AD, DD1] Let $G=F+i H$ with $F, H \in H^{3}(X, \mathbb{R})$, and let $\left\langle W_{G}(z, \tau), \Omega_{z} \wedge\right.$ $\left.\omega_{\tau}\right\rangle=\int_{X}(F+\tau H) \wedge \Omega_{z}$ be the associated superpotential. If $\nabla_{z, \tau} W_{G}(z, \tau)=0$, then $(F+\tau H) \in$ $H_{z}^{2,1} \oplus H_{z}^{0,3}$. Moreover, the map

$$
I_{\tau}: H^{3}(X, \mathbb{C}) \rightarrow H^{3}(X, \mathbb{C}), \quad I_{\tau}(F+i H)=F+\tau H
$$

restricts to give real linear isomorphisms

$$
I_{z, \tau}: H_{z, \tau}^{3} \rightarrow H_{z}^{2,1}(X) \oplus H_{z}^{0,3}(X),
$$

of real vector spaces.

Proof. We first prove that $(F+i H) \mapsto F+\tau H$ takes $H_{Z}^{3} \mapsto H_{z}^{2,1} \oplus H_{z}^{0,3}$. Suppose that $\nabla W_{G}=0$. Since the $\chi_{j}(z)$ span $H_{z}^{2,1}$, we conclude from the first equation of (66) that $(F+\tau H)_{z}^{1,2}=0$; by the second equation, we also have $(F+\tau H)_{z}^{3,0}=0$. Thus $F+\tau H \in$ $H_{z}^{2,1} \oplus H_{z}^{0,3}$.

Since $I_{z, \tau}$ is injective and since $\operatorname{dim}_{\mathbb{R}} H_{z, \tau}^{3}=\operatorname{dim}_{\mathbb{R}} H_{z}^{2,1} \oplus H_{z}^{0,3}=b_{3}$, it is clearly an isomorphism.

3.4. The map $(z, \tau) \rightarrow H_{z, \tau}^{3}$. As $(z, \tau)$ varies over $\mathcal{C}$, how do the spaces $H_{z, \tau}^{3}$ move in $H^{3}(X, \mathbb{C})$ ? This question is important in relating the pure lattice point problem in $H^{3}(X, \mathbb{C})$ to the vacuum distribution problem in $\mathcal{C}$. It depends on the geometry of the diagram

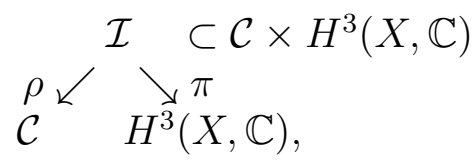

where $\mathcal{I}=\left\{(z, \tau, F, H): F+i H \in H_{(z, \tau)}^{3}(X)\right\}$, which is a replica of (15) in which $\mathcal{S}$ is replaced by $H^{3}(X, \mathbb{C})$.

To answer this question, we first note that for each $(z, \tau) \in \mathcal{C}$, the real-linear map

$$
H_{z, \tau}^{3} \rightarrow H^{3}(X, \mathbb{R}), \quad F+i H \mapsto H
$$

is bijective. Injectivity follows by noting that

$$
F \in H_{z, \tau}^{3} \Longrightarrow F \in H_{z}^{2,1} \oplus H_{z}^{0,3} \Longrightarrow F=\bar{F} \in H_{z}^{1,2} \oplus H_{z}^{3,0} \Longrightarrow F=0
$$

Since both spaces have dimension $b_{3}$, bijectivity follows. Thus there is a real linear isomorphism $\iota_{z, \tau}: H^{3}(X, \mathbb{R}) \rightarrow H_{z, \tau}^{3}$ of the form

$$
\iota_{z, \tau}(H)=F(z, \tau, H)+i H .
$$

To describe $F(z, \tau, H)$, we form the $z$-dependent basis

$$
\left\{\operatorname{Re} D_{1} \Omega_{z}, \ldots, \operatorname{Re} D_{h^{2,1}} \Omega_{z}, \operatorname{Re} \Omega_{z}, \operatorname{Im} D_{1} \Omega_{z}, \ldots, \operatorname{Im} D_{h^{2,1}} \Omega_{z},-\operatorname{Im} \Omega_{z}\right\}
$$

of $H^{3}(X, \mathbb{R})$. We then have

$$
F(z, \tau, H)=J_{\tau} H
$$


where $J_{\tau}$ is given by the block matrix

$$
J_{\tau}=\left(\begin{array}{cc}
\operatorname{Re} \tau I_{m} & -\operatorname{Im} \tau I_{m} \\
\operatorname{Im} \tau I_{m} & \operatorname{Re} \tau I_{m}
\end{array}\right) \quad\left(m=h^{2,1}+1\right),
$$

with respect to the basis (69).

This yields the following proposition:

Proposition 3.2. The mapping $(z, \tau, H) \mapsto\left(z, \tau, \iota_{z, \tau}(H)\right)$ gives an isomorphism $\mathcal{C} \times H^{3}(X, \mathbb{R}) \simeq \mathcal{I}$.

An important consequence is:

Proposition 3.3. For any open subset $U \subset \mathcal{C}$, the cone $\bigcup_{(z, \tau) \in U} H_{(z, \tau)}^{3}(X) \backslash\{0\}$ is open in $H^{3}(X, \mathbb{C}) \backslash\{0\}$.

Proof. We must show that

$$
\pi\left[\mathcal{I} \cap\left\{U \times H^{3}(X, \mathbb{C})\right\}\right] \backslash\{0\}
$$

is open. By Proposition 3.2, it suffices to show that the image of the map

$$
\iota: U \times\left[H^{3}(X, \mathbb{R}) \backslash\{0\}\right] \rightarrow H^{3}(X, \mathbb{C}), \iota(z, \tau, H)=\iota_{z, \tau}(H)=F(z, \tau, H)+i H,
$$

is open. We fix $\left(z_{0}, \tau_{0}, H_{0}\right)$ and consider the derivative $\left.D \iota\right|_{z_{0}, \tau_{0}, H_{0}}$ on $T_{z_{0}, \tau_{0}} \mathcal{C} \times H^{3}(X, \mathbb{R})$. since the linear map $\iota_{z, \tau}$ is bijective, if we vary $H$, we get all of $H_{z, \tau}^{3}$, so the issue is to prove that we obtain the complementary space by taking variations in $\tau, z$.

First, $H_{z, \tau}^{3}=I_{\tau}^{-1}\left(H_{z}^{2,1} \oplus H_{z}^{0,3}\right)$. The $z$ variations of $H_{z}^{2,1} \oplus H_{z}^{0,3}$ span this space plus $H_{z}^{1,2}$. By (69) - (71), variations in $\operatorname{Re} \tau$, resp. $\operatorname{Im} \tau$, produce $\operatorname{Re} \Omega_{z}, \operatorname{Im} \Omega_{z}$ and hence $H_{z}^{3,0}=\operatorname{span}\left(\Omega_{z}\right)$ is also in the image.

Remark: We could also ask what kind of set is swept out in $\bigcup_{z \in U} H_{z}^{2,1} \oplus H_{z}^{0,3}$ as $z$ ranges over an open set $U \subset \mathcal{M}$. Since $\operatorname{dim}_{\mathbb{C}} U=h^{2,1}$, the image of this map is a real codimension two submanifold.

3.5. Inner product on $\mathcal{S}_{Z}$. In Theorem 1.4 we have expressed $\mathcal{N}_{\psi}(L)$ in terms of a Gaussian type ensemble of holomorphic sections in $\mathcal{S}_{Z}$. We now specify the inner product, Gaussian measure and Szegö kernel on this space.

Proposition 3.4. The Hodge-Riemann Hermitian inner product on $H^{3}(X, \mathbb{C})$ restricts for each $Z=(z, \tau)$ to define a complex valued inner product on $H_{Z}^{3}$ which satisfies $Q_{Z}[G]>0$ for all $G \neq 0$. Moreover, the map $I_{\tau}: H_{Z}^{3} \rightarrow H_{z}^{2,1} \oplus H_{z}^{0,3}$ satisfies $Q\left[I_{\tau} G\right]=\operatorname{Im} \tau Q[G]$.

Proof. It follows by Proposition 2.1 that the symmetric bilinear form

$$
Q[F+\tau H]=i^{3} \int_{X}(F+\tau H) \wedge \overline{(F+\tau H)}=\operatorname{Im} \tau Q[F+i H]
$$

on $H_{z, \tau}^{3}(X, \mathbb{C})$ in $(67)$ is positive definite. 
Recall that we have the real-linear isomorphisms

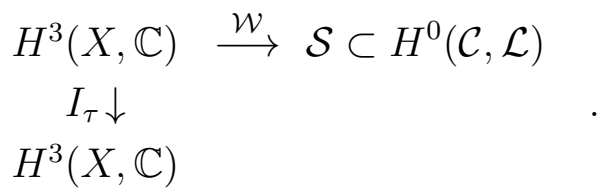

where $I_{\tau}(F+i H)=F+\tau H$. Restricting (73) to fluxes with a critical point at $Z=(z, \tau)$, we have isomorphisms

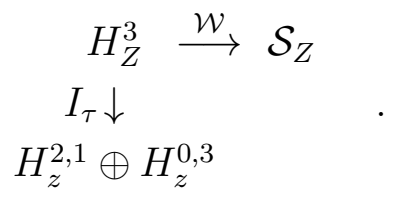

We let $\widetilde{Q}$ denote the Hermitian inner product on $H_{z}^{2,1} \oplus H_{z}^{0,3}$ transported from $\left(H_{Z}^{3}, Q\right)$ by $I_{\tau}$; i.e.,

$$
\widetilde{Q}[C]=Q\left[I_{\tau}^{-1}(C)\right], \quad C \in H_{z}^{2,1} \oplus H_{z}^{0,3} .
$$

Hence by (72), we have:

$$
Q[C]=(\operatorname{Im} \tau) \widetilde{Q}[C]
$$

\section{Counting CRitical Points: Proof of Proposition 1.3}

We now prove the first result on counting critical points of flux superpotentials $W_{G}$ where $G$ satisfies the tadpole constraint (II). Before starting the proof, we review the geometry of the lattice point problem and the critical point problem.

We wish to count vacua in a region of moduli space as $G$ varies over fluxes satisfying the tadpole constraint. Equivalently, we count inequivalent vacua in Teichmüller space. That is, $\Gamma$ acts on the pairs $(W, Z)$ of superpotentials and moduli by

$$
\gamma \cdot(G, Z)=(\varphi(\gamma) \cdot G, \gamma \cdot Z)
$$

Therefore $\Gamma$ acts on the incidence relation (14). We only wish to count critical points modulo the action of $\Gamma$. To do this, there are two choices: we could break the symmetry by fixing a fundamental domain $\mathcal{D}_{\Gamma} \subset \mathcal{C}$ for $\Gamma$ in $\mathcal{C}$, i.e. only count critical points in a fundamental domain. Or we could fix a fundamental domain for $\varphi(\Gamma)$ in $H^{3}(X, \mathbb{C})$ and count all critical points of these special flux superpotentials. When we do not know the group $\varphi(\Gamma)$ precisely, it seems simpler to take the first option and that is what we do in Proposition 1.3 and Theorem 1.4. We note that the number of critical points of $W_{G}$ in Teichmüller space equals the number of critical points of the $\Gamma$-orbit of $W_{G}$ in $\mathcal{C}$.

The level sets $Q[G]=C$ for $C>0$ are hyperboloids contained in $\{G: Q[G]>0\}$ and thus the tadpole constraint defines a hyperbolic shell in $\{G: Q[G]>0\}$. The critical point equation $\nabla W_{G}(Z)=0$ is homogeneous of degree 1 in $G$. Hence, summing a homogeneous function over $G \in\{G: Q[G]>0\}$ with $Q[G] \leq L$ may be viewed as summing a function on the hyperboloid $Q[G]=1$ over the radial projections of the lattice points $G$ in the shell $Q[G] \leq L$. The number which project over a compact subset of $Q[G]=1$ is finite. 
4.1. Approximating the sum by an integral. Our main argument in the proof of Proposition 1.3 is the following lemma:

Lemma 4.1. Let $\psi=\chi_{K}$ where $K \subset \mathcal{I}$ is as in Proposition 1.3. Then

$$
\mathcal{N}_{\psi}(L)=L^{b_{3}}\left[\int_{\mathcal{S}}\left\langle C_{W}, \psi\right\rangle_{Q}(W) d W+O\left(L^{-1 / 2}\right)\right] .
$$

Proof. We consider the integer-valued function

$$
f(W)=\left\langle C_{W}, \psi\right\rangle=\sum_{\{Z: \nabla W(Z)=0\}} \psi(Z, W)=\#\{Z \in \mathcal{C}:(Z, W) \in K\} .
$$

We note that the characteristic function of the set $\{0 \leq Q[W] \leq L\}$ is $\chi_{Q}(W / \sqrt{L})$. Using our symplectic basis to identify $H^{3}(X, \mathbb{Z} \oplus \sqrt{-1} \mathbb{Z})$ with $\mathbb{Z}^{2 b_{3}}$, we have

$$
\mathcal{N}_{\psi}(L)=\sum_{N \in \mathbb{Z}^{2 b_{3}}} f(N) \chi_{Q}(N / \sqrt{L})=\sum_{N \in \mathbb{Z}^{2 b_{3}}} f(N / \sqrt{L}) \chi_{Q}(N / \sqrt{L})=\sum_{N \in \mathbb{Z}^{2 b_{3}}} g(N / \sqrt{L}),
$$

where

$$
g=f \chi_{Q} .
$$

We note that $f$ is constant on each connected component of $\mathcal{S} \backslash[\mathcal{D} \cup \pi(\partial K)]$. Since the number of these components is finite, $f$ is bounded. We let $S\left(\mathcal{S}_{Z}\right)=\left\{N \in \mathcal{S}_{Z}:\|N\|=1\right\}$, where $\|N\|$ denotes the norm in $\mathbb{Z}^{2 b_{3}}$. Since $Q_{Z}$ is positive definite, the sphere $S\left(\mathcal{S}_{Z}\right)$ is contained in the interior of the cone $\{W \in \mathcal{S}: Q[W] \geq 0\}$. Let

$$
A_{\psi}=\sup _{Z \in \rho(\operatorname{Supp} \psi)}\left\|Q_{Z}^{-1}\right\|<+\infty
$$

Then

Now let

$$
\inf \left\{Q[W]: W \in \bigcup_{Z \in \rho(\operatorname{Supp} \psi)} S\left(\mathcal{S}_{Z}\right)\right\}=1 / A_{\psi}>0
$$

$$
Q_{0}:=\left\{W: Q[W] \leq 1,|W| \leq A_{\psi}\right\} \supset \operatorname{Supp} g .
$$

Approximating sums by integrals, we have

$$
L^{-b_{3}} \mathcal{N}_{\psi}(L)=L^{-b_{3}} \sum_{N \in \mathbb{Z}^{2 b_{3}}} g(N / \sqrt{L})=\int_{\mathbb{R}^{2 b_{3}}} g(W) d W+\sum_{N \in \mathbb{Z}^{2 b_{3}}} E_{N, L}
$$

where

$$
\begin{aligned}
E_{N, L}= & \int_{\mathcal{R}_{N, L}}[g(N / \sqrt{L})-g(W)] d W \\
& \mathcal{R}_{N, L}=\left\{W=\left(W_{1}, \ldots, W_{2 b_{3}}\right) \in \mathbb{R}^{2 b_{3}}: N_{j}<W_{j}<N_{j}+1 / \sqrt{L}\right\} .
\end{aligned}
$$

Let

$$
B=Q_{0} \cap[\partial Q \cup \mathcal{D} \cup \pi(\partial K)] .
$$

Since $g$ is locally constant on $\mathcal{S} \backslash B$, the error $E_{N, L}$ vanishes whenever $\mathcal{R}_{N, L} \cap B=\emptyset$. Hence

$$
\sum_{N \in \mathbb{Z}^{2 b_{3}}} E_{N, L} \leq(\sup f) L^{-b_{3}}\left[\#\left\{N: \mathcal{R}_{N, L} \cap B \neq \emptyset\right\}\right]=L^{-b_{3}} O\left(\sqrt{L}^{2 b_{3}-1}\right)=O\left(L^{-1 / 2}\right) .
$$


4.1.1. The index density. By applying precisely the same $\operatorname{argument}$ for $\operatorname{Ind} d_{\psi}(L)$, we obtain Lemma 4.2. Let $\psi=\chi_{K}$ where $K \subset \mathcal{I}$ is as in Proposition 1.3. Then

$$
\mathcal{I} n d_{\psi}(L)=L^{b_{3}}\left[\int_{\{Q[W] \leq 1\}}\left\langle\operatorname{Ind} d_{W}, \psi\right\rangle d W+O\left(L^{-1 / 2}\right)\right] .
$$

4.1.2. Non-clustering of critical points. Before concluding the proof of Proposition 1.3. we briefly consider the question of whether there exist real hypersurfaces $\Gamma \subset \mathcal{C}$ with the property that $\sim \sqrt{L}^{2 b_{3}-1}$ critical points of norm $\leq L$ cluster within a $1 / L$ tube around $\Gamma$. A domain in $\mathcal{C}$ whose boundary contained a piece of $\Gamma$ would attain the remainder estimate in Proposition 1.3 .

Since the number of critical points corresponding to $G \in H^{3}(X, \mathbb{Z} \oplus \sqrt{-1} \mathbb{Z})$ is bounded, such clustering of critical points could only occur if a sublattice of rank $2 b_{3}-1$ clustered around the hypersurface

$$
\bigcup_{(z, \tau) \in \Gamma} H_{z, \tau}^{3} \subset H^{3}(X, \mathbb{C}) .
$$

There do exist real hypersurfaces in $H^{3}(X, \mathbb{C})$ for which such exceptional clustering occurs, namely hyperplanes containing a sublattice of rank $2 b_{3}-1$. We refer to such a hyperplane as a rational hyperplane $L$. For instance, any pair of integral cycles $\gamma_{1}, \gamma_{2}$ defines a rational hyperplane

$$
L=L_{\gamma_{1}, \gamma_{2}}=\left\{G=F+i H \in H^{3}(X, \mathbb{C}): \ell(F+i G):=\int_{\gamma_{1}} F+\int_{\gamma_{2}} H=0\right\} .
$$

As mentioned in the introduction, projections of the lattice points $H^{3}(X, \mathbb{Z} \oplus \sqrt{-1} \mathbb{Z})$ to $\partial Q$ concentrate to sub-leading order $\sqrt{L}^{2 b_{3}-1}$ around the hypersurface of $\partial Q$ obtained by intersecting it with a rational hyperplane.

However, rational hyperplanes never have the form (80). Indeed, under the correspondence $\rho \circ \pi^{*}$ defined by the diagram (68), the image of a hyperplane always covers a region and not a hypersurface of $\mathcal{C}$. That is,

$$
\operatorname{dim}\left(L \cap H_{z, \tau}^{3}\right)>1 \quad \forall(z, \tau) \in \mathcal{C} .
$$

Indeed, under the identification $H_{z, \tau}^{3} \simeq H^{3}(X, \mathbb{R}),\left.L\right|_{H_{z, \tau}^{3}}$ becomes the real linear functional $L(H)=\int_{\gamma_{1}} F(z, \tau, H)+\int_{\gamma_{2}} H$ on $H^{3}(X, \mathbb{R})$. Here, we use that $F(z, \tau, H)$ is linear in $H$. Hence, $\operatorname{dim} L \cap H_{z, \tau}^{3} \geq b_{3}-1$ for any $(z, \tau)$.

As will be studied in [Ze2], clustering to order $\sqrt{L}^{2 b_{3}-1}$ can only occur if the second fundamental form of (80) is completely degenerate. Hence the fact that rational hyperplanes never have this form is strong evidence that there are no smooth hypersurfaces $\Gamma \subset \mathcal{C}$ for which lattice points cluster to subleading order around (80). 
4.2. Hessians and density of critical points. The final step in the proof of Proposition 1.3 is to change the order of integration over $\mathcal{C}$ and over $\mathcal{S}_{Z}$ :

Lemma 4.3. We have:

$$
\int_{\{Q[W] \leq 1\}}\left\langle C_{W}, \psi\right\rangle d W=\int_{\mathcal{C}} \int_{\mathcal{S}_{Z}} \psi(Z, W)\left|\operatorname{det} H^{c} W(Z)\right| \chi_{Q_{Z}}(W) d W d \operatorname{Vol}_{W P}(Z) .
$$

Combining the formulas in Lemmas 4.1 and 4.3. we obtain the formula of Proposition 1.3

The proof of Lemma 4.3 is in two parts. The first is an elementary exercise in changing variables in an integral, which we accomplish below by relating both sides to pushforwards from the incidence variety in the diagram (15). The second part involves special geometry, and is given in the next section.

We may interpret the integral

$$
\int_{\{Q[W] \leq 1\}}\left\langle C_{W}, \psi\right\rangle d W
$$

as an integral over $\mathcal{I}$ as follows. Implicitly, it defines a measure $d \mu_{\mathcal{I}}$ so that

$$
\int_{\mathcal{I}} \psi(Z, W) d \mu_{\mathcal{I}}=\int_{\{Q[W] \leq 1\}}\left\langle C_{W}, \psi\right\rangle d W .
$$

The measure $d \mu_{\mathcal{I}}$ may be expressed in terms of the Leray measure $d \mathcal{L}_{\mathcal{I}}$ defined by a measure $d \nu$ on $\mathcal{S}$ and the 'evaluation map'

$$
\varepsilon:(Z, W) \in \mathcal{C} \times \mathcal{S} \rightarrow \nabla W(Z) .
$$

The Leray form is the quotient $d \mathcal{L}_{\mathcal{I}}:=\frac{d V_{W P} \times d \nu}{d \varepsilon}$, i.e. the unique form satisfying

$$
d \mathcal{L}_{\mathcal{I}} \times d \varepsilon=d V_{W P} \times d \nu .
$$

This measure is often written $\delta(\nabla W(Z)) d W d V(Z)$ in the physics literature.

As suggested by the physics formula, $d \mu_{\mathcal{I}}=\nabla s(Z)^{*} \delta_{0}$. However, this formula is somewhat ambiguous. If we regard $s$ as fixed, then it is simply the pullback of $\delta_{0}$ under $Z \rightarrow \nabla s(Z)$. It is then well-known that

$$
\nabla s^{*} \delta_{0}=\sum_{Z: \nabla s(Z)=0} \frac{\delta_{Z}}{\left|\operatorname{det} H^{c} s(Z)\right|} .
$$

However, when interchanging the order of integration, we really wish to think of it as a function of $s$ for fixed $Z$. So we now have a function $\varepsilon_{Z}(s)=\nabla s(Z)$ which may be viewed as

$$
\varepsilon_{Z}: \mathcal{S} \rightarrow \mathbb{C}^{m} \equiv \mathbb{R}^{b_{3}}
$$

where $m=h^{2,1}+1=\frac{1}{2} b_{3}$. So now the zero set $\varepsilon_{Z}^{-1}(0)$ is the subspace $\mathcal{S}_{Z}$ rather than the discrete set $\operatorname{Crit}(s)$.

To simplify the notation, we now consider the general situation where we have a real $n$ dimensional manifold $M$ and a space $\mathcal{S}$ of functions $F: M_{n} \rightarrow \mathbb{R}^{n}$. In our case, $F=\nabla s$ and $M$ is a coordinate neighborhood in $\mathcal{C}$ where $M$ has local coordinates $\left(x_{1}, \ldots, x_{n}\right)$ and $\mathcal{L}$ has a local frame. Suppose that 0 is a regular value of $F$, so that $F$ is a local diffeomorphism in a neighborhood $U$ of any point $x_{0}$ of $F^{-1}(0)$. Let $h=F_{\mid U}^{-1}$ in a neighborhood of 0 . Then for $\varphi$ supported in a neighborhood of $x_{0}$, put

$$
\left\langle F^{*} \delta_{0}, \varphi\right\rangle=\left\langle\delta_{0}, \varphi(h(y))|\operatorname{det} d h(y)|\right\rangle .
$$


Let $\operatorname{dim}_{\mathbb{R}} \mathcal{S}=d \geq n$. In our case, $d=2 b_{3}>n=b_{3}$, so we introduce a supplementary linear map: for a point $u \in U \subset M, \mathcal{S}_{u}$ is the kernel of $\varepsilon_{u}$, and we supplement $\varepsilon_{u}$ with the projection $\Pi_{u}: \mathcal{S} \rightarrow \mathcal{S}_{u}$. Then,

$$
\left(\varepsilon_{u}, \Pi_{u}\right): \mathcal{S} \rightarrow \mathbb{R}^{n} \oplus \mathcal{S}_{u}
$$

is a linear isomorphism. Hence it equals its derivative, so

$$
\left\langle\varepsilon_{u}^{*} \delta_{0}, \varphi\right\rangle=\left\langle\delta_{0}, \varphi\left(\left(\varepsilon_{u}, \Pi_{u}\right)^{-1}\right)\left|\operatorname{det}\left(\varepsilon_{u}, \Pi_{u}\right)^{-1}\right|\right\rangle .
$$

Now, $\mathcal{S}$ is equipped with an inner product, which induces an inner product on $\mathbb{R}^{n} \oplus \mathcal{S}_{u}$. We choose an orthonormal basis $\left\{S_{1}, \ldots, S_{n}\right\}$ of $\mathcal{S}_{u}^{\perp}$, and $\left\{S_{n+1}, \ldots, S_{d}\right\}$ for $\mathcal{S}_{u}$. Since $\Pi_{u}: \mathcal{S}_{u} \rightarrow$ $\mathcal{S}_{u}$ is the identity, $\left(\varepsilon_{u}, \Pi_{u}\right)$ has a block diagonal matrix relative to the bases of $\mathcal{S}=\mathcal{S}_{u}^{\perp} \oplus \mathcal{S}_{u}$ and $\mathbb{R}^{n} \oplus \mathcal{S}_{u}$, with the identity in the $\mathcal{S}_{u}-\mathcal{S}_{u}$ block. Hence, $\operatorname{det}\left(\varepsilon_{u}, \Pi_{u}\right)=\operatorname{det}\left(\left.\varepsilon_{u}\right|_{\mathcal{S}^{\perp}}\right)$ where the determinant is with respect to these bases.

The general case of formula (81) states that

$$
d \mu_{\mathcal{I}}=|\operatorname{det} D W(u)| \times \frac{\chi_{Q} d u \times d W}{d \varepsilon} .
$$

We then compute the $\mathcal{I}$ integral as an iterated integral using the other singular fibration $\pi$, i.e. by first integrating over the fibers $\mathcal{S}_{u}$ :

$$
\int_{\mathcal{I}} \psi(u) d \mu_{\mathcal{I}}=\int_{U} \int_{\mathcal{S}_{u}} \frac{\psi(u)}{\left|\operatorname{det}\left(\varepsilon_{u} \mid \mathcal{S}_{u}^{\perp}\right)\right|} \chi_{Q_{u}}(W)|\operatorname{det} D W(u)| d W d u .
$$

Returning to our case where $F=\nabla s$, (184) becomes

$$
\int_{\mathcal{I}} \psi(Z) d \mu_{\mathcal{I}}=\int_{\mathcal{C}} \int_{\mathcal{S}_{Z}} \frac{\psi(Z, W)}{\left|\operatorname{det}\left(\left.\varepsilon_{Z}\right|_{\mathcal{S}_{Z}^{\perp}}\right)\right|}\left|\operatorname{det} H^{c} W(Z)\right| \chi_{Q_{Z}}(W) d W d \operatorname{Vol}_{W P}(Z) .
$$

4.3. Completion of the proof of Lemma 4.3. To complete the proof of the lemma, we need to show that $\left|\operatorname{det}\left(\left.\varepsilon_{Z}\right|_{\mathcal{S}_{Z}^{\perp}}\right)\right|=1$ with respect to normal coordinates and an adapted frame at $Z_{0}=\left(z_{0}, \tau_{0}\right) \in M$.

Recalling (73) $-(74)$, we write

$$
\widetilde{\mathcal{S}}_{Z}^{\perp}=I_{\tau} \circ \mathcal{W}^{-1}\left(\mathcal{S}_{Z}^{\perp}\right)=H_{z}^{3,0} \oplus H_{z}^{1,2}
$$

A complex orthonormal basis for $\widetilde{\mathcal{S}}_{Z_{0}}^{\perp}$ relative to $Q$ is $\left\{\bar{\chi}_{0}, \bar{\chi}_{1}, \ldots, \bar{\chi}_{h^{2,1}}\right\}$, where $\chi_{0}=\bar{\Omega}_{z_{0}}$. A basis (over $\mathbb{R}$ ) for $\mathcal{S}_{Z_{0}}^{\perp}$ is

$$
\bar{U}_{j}:=\mathcal{W} \circ I_{\tau}^{-1}\left(\bar{\chi}_{j}\right), \quad \bar{V}_{j}:=\mathcal{W} \circ I_{\tau}^{-1}\left(\sqrt{-1} \bar{\chi}_{j}\right), \quad 0 \leq j \leq h^{2,1} .
$$

The basis $\left\{\bar{U}_{j}, \bar{V}_{j}\right\}$ is orthogonal with respect to $Q_{Z_{0}}$, but not orthonormal. By (76)

$$
Q\left[\bar{U}_{j}\right]=\widetilde{Q}\left[\bar{\chi}_{j}\right]=\frac{1}{\operatorname{Im} \tau} Q\left[\bar{\chi}_{j}\right]=\frac{1}{\operatorname{Im} \tau}, \quad Q\left[\bar{V}_{j}\right]=\widetilde{Q}\left[\sqrt{-1} \bar{\chi}_{j}\right]=\frac{1}{\operatorname{Im} \tau} .
$$

To compute $\operatorname{det}\left(\varepsilon_{Z_{0}} \mid \mathcal{S}_{Z_{0}}^{\perp}\right)$, we let $\left(z_{1}, \ldots, z_{h^{2,1}}\right)$ be normal coordinates about $z_{0} \in \mathcal{M}$, and we let $\nabla_{j} f$ be given by

$$
\nabla_{\partial / \partial z^{j}}\left(f e_{\mathcal{L}}\right)=\left(\nabla_{j} f\right) \otimes e_{\mathcal{L}}
$$

for $1 \leq j \leq h^{2,1}$. We find it convenient to use the coordinate $\tau \in \mathcal{E}$, although it is not normal, and we use the normalized covariant derivative

$$
\nabla_{0}:=(\operatorname{Im} \tau) \nabla_{\tau}
$$


Now we write

$$
\bar{U}_{j}=f_{j}(z) \Omega_{z}^{*} \otimes \omega_{\tau}^{*}, \quad \bar{V}_{j}=g_{j}(z) \Omega_{z}^{*} \otimes \omega_{\tau}^{*},
$$

where the local frame $\Omega_{z}$ is normal at $z_{0}$, and $\omega_{\tau}=d x+\tau d y$. Note that the Weil-Petersson norm $\left|\omega_{\tau}^{*}\right|$ is given by

$$
\left|\omega_{\tau}^{*}\right|=|d x+\tau d y|^{-1}=\frac{1}{(\operatorname{Im} \tau)^{1 / 2}} .
$$

Taking into account (86)-(88), the $\operatorname{Im} \tau$ factors cancel out, and we obtain

$$
\left.\operatorname{det}\left(\varepsilon_{Z_{0}} \mid \mathcal{S}_{Z_{0}}^{\perp}\right)\right)=\left.\operatorname{det}\left(\begin{array}{cc}
\operatorname{Re} \nabla_{j} f_{k} & \operatorname{Re} \nabla_{j} g_{k} \\
\operatorname{Im} \nabla_{j} f_{k} & \operatorname{Im} \nabla_{j} g_{k}
\end{array}\right)\right|_{Z_{0}}, \quad \text { for } \quad 0 \leq j, k \leq h^{2,1}
$$

We now evaluate the entries of the matrix. By Proposition 2.2, we have

$$
\nabla_{k} f_{j}(Z)=\int_{X} \overline{\mathcal{D}_{j} \Omega_{z_{0}}} \wedge \mathcal{D}_{k} \Omega_{z}, \quad \nabla_{k} g_{j}(Z)=\int_{X} i \overline{\mathcal{D}_{j} \Omega_{z_{0}}} \wedge \mathcal{D}_{k} \Omega_{z}
$$

and hence

$$
\nabla_{j} f_{k}\left(Z_{0}\right)=-i \delta_{j k}, \quad \nabla_{j} g_{k}\left(Z_{0}\right)=\delta_{j k}, \quad \text { for } j, k \geq 1
$$

Also

$$
\nabla_{k} f_{0}=\int_{X} \Omega_{z_{0}} \wedge\left[\mathcal{D}_{k} \Omega_{z_{0}}-\left(\partial K / \partial z_{j}\right) \Omega_{z_{0}}\right]=0, \quad \nabla_{k} g_{0}=i \nabla_{k} f_{0}=0 \quad \text { for } k \geq 1 .
$$

By (64), we have

$$
\nabla_{0}\left(f_{j}\right)=(\operatorname{Im} \tau) \nabla_{\tau}\left(f_{j}\right)=\int_{X} \mathcal{D}_{j} \Omega_{z_{0}} \wedge \Omega_{z_{0}}=0, \quad \nabla_{0}\left(g_{j}\right)=-i \int_{X} \Omega_{z_{0}} \wedge \Omega_{z_{0}}=0, \quad j \geq 1,
$$

and

$$
\nabla_{0}\left(f_{0}\right)=\int_{X} \overline{\Omega_{z_{0}}} \wedge \Omega_{z_{0}}=i, \quad \nabla_{0}\left(g_{0}\right)=\int_{X} \overline{i \Omega_{z_{0}}} \wedge \Omega_{z_{0}}=1
$$

Therefore,

$$
\left.\mid \operatorname{det}\left(\varepsilon_{Z_{0}} \mid \mathcal{S}_{Z_{0}}^{\perp}\right)\right)|=| \operatorname{det}\left(\begin{array}{cc}
0 & I \\
D(1,-1, \ldots,-1) & 0
\end{array}\right) \mid=1
$$

\section{Proof of Theorem 1.4}

In this section we prove Theorem 1.4, which is a combination of an equidistribution theorem for radial projections of lattice points and an equidistribution theorem for critical points. 
5.1. A local van der Corput Theorem. We first illustrate the method of proof of Theorem 1.4 by providing a van der Corput type asymptotic estimate for the radial distribution of lattice points (Theorem 5.1). The estimate has much in common with the classical van der Corput estimate of Hlawka, Randol and others on lattice points in dilates of smooth convex sets (see for example, $[\mathrm{Ra}, \mathrm{Hl}]$ ), and we adapt the proof of the classical estimate to obtain our asymptotic equidistribution theorem.

Let $Q \subset \mathbb{R}^{n}(n \geq 2)$ be a bounded, smooth, strictly convex set with $0 \in Q^{\circ}$. Let $|X|_{Q}$ denote the norm of $X \in \mathbb{R}^{n}$ given by

$$
Q=\left\{X \in \mathbb{R}^{n}:|X|_{Q}<1\right\} .
$$

To measure the equidistribution of projections of lattice points, we consider the sums

$$
S_{f}(t)=\sum_{k \in \mathbb{Z}^{n} \cap t Q \backslash\{0\}} f\left(\frac{k}{|k|_{Q}}\right) \text {, with } f \in C^{\infty}(\partial Q), t>0 .
$$

We extend $f$ to $\mathbb{R}^{n}$ as a homogeneous function of degree 0 , so that $f(k)=f\left(\frac{k}{|k|_{Q}}\right)$. Our purpose is to obtain the following asymptotics of $S_{f}(t)$ :

THEOREM 5.1.

$$
S_{f}(t)=t^{n} \int_{Q} f d X+O\left(t^{n-\frac{2 n}{n+1}}\right), \quad t \rightarrow \infty .
$$

From this it is simple to obtain asymptotics of $S_{f}(t)$ when $f \in C^{\infty}(\partial Q)$ is extended as a homogeneous function of any degree $\alpha$ to $\mathbb{R}^{n}$ :

Corollary 5.2. Let $f \in \mathcal{C}^{\infty}\left(\mathbb{R}^{n} \backslash\{0\}\right)$ be homogeneous of degree $\alpha>0$, and let

$$
S_{f}(t)=\sum_{k \in \mathbb{Z}^{n} \cap t Q} f(k), \quad t>0
$$

Then

$$
S_{f}(t)=t^{n+\alpha} \int_{Q} f d X+O\left(t^{n-\frac{2 n}{n+1}+\alpha}\right), \quad t \rightarrow \infty .
$$

5.1.1. Littlewood-Paley. To deal with the singularity of $f$ at $x=0$ we use a dyadic LittlewoodPaley decomposition in the radial direction. Let $\eta \in C_{0}^{\infty}$ with $\eta(r)=1$ for $r \leq 1$ and with $\eta(r)=0$ for $r \geq 2$. We then define

$$
\rho \in C_{0}^{\infty}(\mathbb{R}), \quad \rho(r)=\eta(r)-\eta(2 r) .
$$

Then $\rho(r)$ is supported in the shell $1 / 2 \leq r \leq 2$, hence $\rho\left(2^{j} r\right)$ is supported in the shell $2^{-j-1} \leq r \leq 2^{-j+1}$. We then have:

$$
\eta(r)=\sum_{j=0}^{\infty} \rho\left(2^{j} r\right), \quad(r \neq 0)
$$

Indeed,

$$
\sum_{j=0}^{J} \rho\left(2^{j} r\right)=\eta(r)-\eta\left(2^{J} r\right) \rightarrow \eta(r)
$$

by the assumption that $\eta \in C_{0}^{\infty}$. 
We then write

$$
\begin{aligned}
& S_{f}(t)= \sum_{k \in \mathbb{Z}^{n}} f(k) \chi_{[0,1]}\left(\frac{|k|_{Q}}{t}\right)=S_{f}^{\prime}(t)+S_{f}^{\prime \prime}(t), \\
& S_{f}^{\prime}(t)=\sum_{k \in \mathbb{Z}^{n}} f(k) \eta\left(\frac{|k|_{Q}}{t}\right), \\
&\left.S_{f}^{\prime \prime}(t)=\sum_{k \in \mathbb{Z}^{n}} f(k)\left(\chi_{[0,1]}-\eta\right)\left(\frac{|k|_{Q}}{t}\right)\right) .
\end{aligned}
$$

We can assume without loss of generality that $f \geq 0$. We begin with the first sum in $S_{f}^{\prime}(t)$ :

\section{LEMMA 5.3.}

$$
S_{f}^{\prime}(t)=t^{n} \int_{\mathbb{R}^{n}} f(X) \eta\left(|X|_{Q}\right) d X+O(\log t) .
$$

Proof. We write the sum as

$$
S_{f}^{\prime}(t)=\sum_{j=0}^{\infty} \sum_{k \in \mathbb{Z}^{n}} f(k) \rho\left(\frac{2^{j}|k|_{Q}}{t}\right) .
$$

We further break up the dyadic sum into $\sum_{j=0}^{J(t)}+\sum_{j=J(t)+1}^{\infty}$ with $J(t)$ to be determined later. We first consider

$$
S_{1}^{\prime}:=\sum_{j=0}^{J(t)} \sum_{k \in \mathbb{Z}^{n}} f(k) \rho\left(\frac{2^{j}|k|_{Q}}{t}\right) .
$$

The function $f(X) \rho\left(2^{j}|X|_{Q}\right) \in C_{0}^{\infty}\left(\mathbb{R}^{n}\right)$ when $f$ is homogeneous of degree 0 and smooth on $\partial Q$. Hence we may apply the Poisson summation formula to the $k$ sum to obtain

$$
S_{1}^{\prime}=\sum_{j=0}^{J(t)} \sum_{N \in \mathbb{Z}^{n}} \int_{\mathbb{R}^{n}} e^{-i\langle X, N\rangle} f(X) \rho\left(\frac{2^{j}|X|_{Q}}{t}\right) d X .
$$

The terms with $N=0$ sum up to

$$
\begin{aligned}
t^{n} \int_{\mathbb{R}^{n}} f(X)\left\{\sum_{j=0}^{J(t)} \rho\left(2^{j}|X|_{Q}\right)\right\} d X & \left.=t^{n} \int_{\mathbb{R}^{n}} f(X)\left\{\eta\left(|X|_{Q}\right)-\eta\left(2^{J(t)+1}|X|_{Q}\right)\right)\right\} d X \\
& =t^{n} \int_{\mathbb{R}^{n}} f(X) \eta\left(|X|_{Q}\right) d X+O\left(t^{n} 2^{-n J(t)}\right),
\end{aligned}
$$

where the last estimate is a consequence of the fact that $\eta\left(2^{J(t)+1}|X|_{Q}\right)$ is supported on $2^{-J(t)} Q$.

To estimate the remaining terms in the sum $S_{1}^{\prime}$, we make the change of variables $Y=2^{j} X / t$ in the integral to obtain

$$
2^{-n j} t^{n} \int_{\mathbb{R}^{n}} f(Y) \rho(|Y|) e^{-i 2^{-j} t\langle Y, N\rangle} d Y
$$

Since the integrand is smooth, this term has the upper bound

$$
c 2^{-n j} t^{n}\left(1+2^{-j}|N| t\right)^{-K}, \quad \forall K>0 .
$$


(Again, we let $c$ denote a constant; $c$ depends on $f$ and $K$, but is independent of $j, t, N$.) The sum over $N \neq 0$ is then bounded by

$$
\begin{aligned}
c t^{n} \sum_{j \leq J(t)} 2^{-n j} \sum_{N \neq 0}\left(1+2^{-j}|N| t\right)^{-K} & \sim t^{n} \sum_{j \leq J(t)} 2^{-n j} \int_{0}^{\infty}\left(1+2^{-j} r t\right)^{-K} r^{n-1} d r \\
& =\sum_{j \leq J(t)} \int_{0}^{\infty}(1+s)^{-K} s^{n-1} d s=c J(t) .
\end{aligned}
$$

Therefore

$$
S_{1}^{\prime}=t^{n} \int_{\mathbb{R}^{n}} f(X) \eta\left(|X|_{Q}\right) d X+O\left(t^{n} 2^{-n J(t)}\right)+O(J(t)) .
$$

Recall that $S_{f}^{\prime}(t)=S_{1}^{\prime}+S_{2}^{\prime}$, where

$$
S_{2}^{\prime}=\sum_{j=J(t)+1}^{\infty} \sum_{k \in \mathbb{Z}^{n}} f\left(\frac{k}{|k|_{Q}}\right) \rho\left(\frac{2^{j}|k|_{Q}}{t}\right) .
$$

Since

$$
\sum_{j=J(t)+1}^{\infty} \rho\left(\frac{2^{j}|k|_{Q}}{t}\right)=\eta\left(\frac{2^{J(t)}|k|_{Q}}{t}\right) \leq \chi_{t 2^{-J(t)} Q}
$$

the remainder $S_{2}^{\prime}$ is bounded by the total number of lattice points in the shell $|k|_{Q} \leq 2^{-J(t)} t$, hence is of order $t^{n} 2^{-n J(t)}$. It follows that

$$
S_{f}^{\prime}(t)=t^{n} \int_{\mathbb{R}^{n}} f(X) \eta\left(|X|_{Q}\right) d X+O\left(t^{n} 2^{-n J(t)}\right)+O(J(t)) .
$$

To balance the terms, we choose $J(t)=\log _{2} t$, and then the last two terms of (92) have the form

$$
O\left(t^{n} t^{-n}\right)+O(\log t)=O(\log t)
$$

5.1.2. Stationary phase. Theorem 5.1 is an immediate consequence of Lemma 5.3 and the following assymptotics of the second sum $S_{f}^{\prime \prime}(t)$ from (91):

LEMMA 5.4.

$$
S_{f}^{\prime \prime}(t)=t^{n} \int_{\mathbb{R}^{n}} f(X)\left(\chi_{[0,1]}-\eta\right)\left(|X|_{Q}\right) d X+O\left(t^{n-\frac{2 n}{n+1}}\right)
$$

Proof. Let

$$
g(X)=f(X)\left(\chi_{[0,1]}-\eta\right)\left(|X|_{Q}\right)
$$

and mollify $g$ by a radial approximate identify $\varphi_{\varepsilon}$ to obtain a smooth approximation $g_{\varepsilon}=$ $g * \varphi_{\varepsilon}$. We claim that

$$
S_{f}^{\prime \prime}(t)=\sum_{k \in \mathbb{Z}^{n}} g\left(\frac{k}{t}\right)=\sum_{k \in \mathbb{Z}^{n}} g_{\varepsilon}\left(\frac{k}{t}\right)+O\left(\varepsilon t^{n}\right) .
$$


To see this, we break the sum into two parts. The first part is over the lattice points $k$ with $k / t$ in an $\varepsilon$ tube $T_{\varepsilon}$ about $\left\{|X|_{Q}=1\right\}$. The number of such $k$ is $O\left(\varepsilon t^{n}\right)$, so this part contributes the stated error. For the remaining sum, the error is

$$
\left|\sum_{k \in \mathbb{Z}^{n} \backslash t T_{\varepsilon}}\left[g\left(\frac{k}{t}\right)-g_{\varepsilon}\left(\frac{k}{t}\right)\right]\right| \leq \sum_{k / t \in \operatorname{Supp} g \backslash T_{\varepsilon}} \varepsilon \sup _{|X|_{Q}>1}|d g(X)|=O\left(\varepsilon t^{n}\right),
$$

which verifies (93).

The Poisson summation formula then gives

$$
\sum_{k \in \mathbb{Z}^{n}} g_{\varepsilon}(k / t)=t^{n} \sum_{N \in \mathbb{Z}^{n}} \hat{g}_{\varepsilon}(2 \pi t N)=t^{n} \sum \hat{g}(2 \pi t N) \hat{\varphi}(2 \pi t \varepsilon N) .
$$

The term $N=0$ yields

$$
t^{N} \int_{\mathbb{R}^{n}} g_{\varepsilon}(X) d X=t^{n} \int_{\mathbb{R}^{n}} f(X)\left(\chi_{[0,1]}-\eta\right)\left(|X|_{Q}\right) d X+O\left(\varepsilon t^{n}\right),
$$

where the last inequality is by breaking up the integral into two parts as above.

As for the remainder terms $N \neq 0$, we now show that

$$
\hat{g}(2 \pi t N)=O\left((1+|t N|)^{-\frac{(n+1)}{2}}\right) .
$$

To verify (94), we write

$$
\begin{aligned}
& g=-f \rho h=-(f \rho)\left(h \eta_{2}\right), \quad \text { with } \quad \eta_{2}(X)=\eta\left(\frac{1}{2}|X|_{Q}\right), \quad h=\theta \circ \lambda, \\
& \lambda(X)=|X|_{Q}-1, \quad \theta(t)=\text { Heaviside function }= \begin{cases}0, & \text { if } t<0 \\
1, & \text { if } t \geq 0 .\end{cases}
\end{aligned}
$$

Since $\widehat{g}=-\widehat{f \rho} * \widehat{h \eta_{2}}$ and $\widehat{f \rho}$ is rapidly decaying, it suffices to show that $\widehat{h \eta_{2}}$ satisfies (94). (Here, we use the elementary estimate $\|\alpha * \beta\|_{(K)} \leq c\|\alpha\|_{(K+n+1)}\|\beta\|_{(K)}$, where $\|\alpha\|_{(K)}=$ $\sup _{x \in \mathbb{R}^{n}}(1+|x|)^{K}|\alpha(x)|$.) Taking partial derivatives,

$$
\mathcal{D}_{j}\left(h \eta_{2}\right)=\mathcal{D}_{j} \eta_{2}+\left(\delta_{0} \circ \lambda\right) \mathcal{D}_{j} \lambda
$$

Since the latter term is given by integration over $\partial Q$, which is strictly convex, the standard stationary phase method (see Hörmander [Ho] $)$ immediately gives $\left(\delta_{0} \circ \lambda\right) \widehat{\Upsilon}(x)=O\left(x^{-\frac{(n-1)}{2}}\right)$, and hence

$$
x_{j} \widehat{h \eta_{2}}=\left[\mathcal{D}_{j}\left(h \eta_{2}\right) \widetilde{\Gamma}=O\left(x^{-\frac{(n-1)}{2}}\right)\right.
$$

which implies (94).

Hence the remainder is bounded above by

$$
c t^{n} \sum_{N \neq 0}(1+|t N|)^{-\frac{(n+1)}{2}}(1+|\varepsilon t N|)^{-K}
$$


The sum (95) can be replaced by the integral

$$
\begin{aligned}
c t^{n} \int_{\mathbb{R}^{n}}(1+|t N|)^{-\frac{(n+1)}{2}}(1+|\varepsilon t N|)^{-K} d N & =c t^{n} \int_{0}^{\infty}(1+t r)^{-\frac{(n+1)}{2}}(1+\varepsilon t r)^{-K} r^{n-1} d r \\
& =c \varepsilon^{\frac{1-n}{2}} \int_{0}^{\infty}(\varepsilon+s)^{-\frac{(n+1)}{2}}(1+s)^{-K} s^{n-1} d s \\
& \leq c \varepsilon^{\frac{1-n}{2}} \int_{0}^{\infty}(1+s)^{-K} s^{\frac{n-3}{2}} d s=c \varepsilon^{\frac{1-n}{2}} .
\end{aligned}
$$

Hence

$$
S_{f}^{\prime \prime}(t)=t^{n} \int_{\mathbb{R}^{n}} f(X)\left(\chi_{[0,1]}-\eta\right)\left(|X|_{Q}\right) d X+O\left(\varepsilon t^{n}\right)+O\left(\varepsilon^{-(n-1) / 2}\right) .
$$

To optimize, we choose $\varepsilon$ so that $\varepsilon t^{n}=\varepsilon^{-(n-1) / 2}$, i.e. $\varepsilon=t^{-2 n /(n+1)}$, which gives the result. (To be precise, it is the sum of the terms in (95) with $|N| \geq \sqrt{n}$ that is bounded by the above integral. But there are only finitely many terms with $|N|<\sqrt{n}$, and each of these terms is $<c t^{n-\frac{n+1}{2}}$, which is better than $O\left(t^{n-\frac{2 n}{n+1}}\right)$ when $n \geq 2$.)

5.1.3. Van der Corput for homogeneous weights $f$. Proof of Corollary 5.2: This time, we have

$$
S_{f}(t)=\sum_{k \in \mathbb{Z}^{n} \cap t Q \backslash\{0\}}|k|_{Q}^{\alpha} f\left(\frac{k}{|k|_{Q}}\right) .
$$

The set of norms of lattice points $\left\{t_{j} \in \mathbb{R}^{+}: \exists k \in \mathbb{Z}^{n} \quad \ni \quad|k|_{Q}=t_{j}\right\}$ is a countable set without accumulation point. We order the $t_{j}$ so that $t_{j} \leq t_{j+1}$. We then define the monotone increasing step function on $\mathbb{R}$

$$
\mu(T)=\sum_{j: t_{j} \leq T}\left\{\sum_{k:|k|_{Q}=t_{j}} f\left(\frac{k}{|k|_{Q}}\right)\right\} .
$$

It is clear that

Hence, by Theorem 5.1 .

$$
\mu(T)=S_{f_{0}}(T), \quad f_{0}(x)=\frac{f(x)}{|x|_{Q}}
$$

$$
S_{f_{0}}(t)=t^{n} \int_{Q} f_{0} d X+O\left(t^{n-\frac{2 n}{n+1}}\right), \quad t \rightarrow \infty .
$$

We further have

$$
S_{f}(T)=\int_{0}^{T} t^{\alpha} d \mu(t)
$$

Indeed,

$$
d \mu(t)=\sum_{j}\left\{\sum_{k:|k|_{Q}=t_{j}} f\left(\frac{k}{|k|_{Q}}\right)\right\} \delta\left(t_{j}\right),
$$

and

$$
\int_{0}^{T} t^{\alpha} d \mu(t)=\sum_{j: t_{j} \leq T}\left\{\sum_{k:|k|_{Q}=t_{j}} f\left(\frac{k}{|k|_{Q}}\right)\right\} t_{j}^{\alpha}=S_{f}(T) .
$$


Integrating (97) by parts and applying (96), we get

$$
\begin{aligned}
S_{f}(T) & =T^{\alpha} \mu(T)-\alpha \int_{0}^{T} t^{\alpha-1} \mu(t) d t \\
& =T^{\alpha}\left[T^{n} \int_{Q} f_{0} d X+O\left(T^{n-\frac{2 n}{n+1}}\right)\right]-\alpha \int_{0}^{T} t^{\alpha-1}\left[t^{n} \int_{Q} f_{0} d X+O\left(t^{n-\frac{2 n}{n+1}}\right)\right] d t \\
& =T^{n+\alpha}\left[\int_{Q} f_{0} d X\right] \frac{n}{\alpha+n}+O\left(T^{n-\frac{2 n}{n+1}+\alpha}\right) \\
& =T^{n+\alpha} \int_{Q} f d X+O\left(T^{n-\frac{2 n}{n+1}+\alpha}\right) .
\end{aligned}
$$

5.2. Van der Corput for critical points. We prove Theorem 1.4 by following the arguments of the proofs of Theorem [5.1 and Corollary 5.2 with hardly any changes. We first assume that $\psi$ is homogeneous of order 0 in $\mathcal{S}$. We let $K_{\psi}=\rho(\operatorname{Supp} \psi) \subset \mathcal{C}$, a compact set.

To begin, we recall that if $W$ has critical points, then $W$ is in the 'light cone' $Q[W]>0$. For $W$ in the light cone, we write

$$
|W|_{Q}=Q[W]^{\frac{1}{2}}, \quad \text { for } Q[W]>0 .
$$

The main difference between this case and our previous one, is that now the set $Q$ given by (89), in addition to not being convex, is not compact. However, since only with those $W$ with critical points in the support of $\psi$ contribute to the sum, we consider

$$
Q_{\psi}:=Q \cap \mathcal{S}_{\psi}, \quad \mathcal{S}_{\psi}=\left(\bigcup_{\tau \in K_{\psi}} \mathcal{S}_{\tau}\right)
$$

which is a compact subset of $\mathcal{S}$.

We let $f(W)=\left\langle C_{W}, \psi\right\rangle$, which is a smooth function supported in $\mathcal{S}_{\psi}$. Then

$$
\mathcal{N}_{\psi}(L)=S_{f}(L)=\sum_{k \in \mathbb{Z}^{n} \cap \sqrt{L} Q \backslash\{0\}} f(k),
$$

as before. Now we follow the previous proof, with $t=\sqrt{L}$. Our first modification is to verify (93), we instead let $T_{\varepsilon}$ be the epsilon tube over $\mathcal{S}_{\psi} \cap \partial Q$. Finally, the estimate $\left(\delta_{0} \circ \lambda\right) \widehat{(}(t)=O\left(t^{-\frac{(n-1)}{2}}\right)$, which was based on the convexity of $Q$ in our previous argument, holds in this case, since the phase $\psi(Y)=L\langle Y, N\rangle$ has (two) non-degenerate critical points whenever $N$ is in the light cone. Thus we have

$$
\mathcal{N}_{\psi}(L)=L^{b_{3}}\left[\int_{\{Q[W] \leq 1\}}\left\langle C_{W}, \psi\right\rangle d W+O\left(L^{-\frac{2 b_{3}}{2 b_{3}+1}}\right)\right] .
$$

The case $\alpha=0$ now follows from Lemma 4.3, and the general case then follows exactly as in the proof of Corollary 5.2 . 


\section{SPECIAL GEOMETRY AND DENSITY OF CRITICAL POINTS}

The aim of this section is to compute the critical point density $\mathcal{K}^{\text {crit }}(Z)$ and verify Corollaries 1.5]1.6] At the same time, we compute the index density and prove Theorem 1.8. As in [DSZ1, we do this by pushing forward the integrand of (25) under the Hessian map. The Hessian map turns out to be an isomorphism, hence the discussion is more elementary than in DSZ1. To make the change of variables, we first evaluate the image of the Hessian using the special geometry of Calabi-Yau moduli spaces and then check how the Hessian map distorts inner products. Our discussion gives an alternate approach to the formulas in the article [DD1], and connects the special critical point density formula in this article with the general ones in DSZ1, DSZ2.

6.1. The range of the Hessian map. We now study the complex Hessian map:

$$
H^{c}(Z): W \rightarrow\left(\begin{array}{cc}
H^{\prime} & -x \Theta(Z) \\
-\bar{x} \bar{\Theta}(Z) & \bar{H}^{\prime}
\end{array}\right) .
$$

To describe $H^{c}(Z)$ in local coordinates, we fix a point $Z_{0}=\left(z_{0}, \tau_{0}\right)$ and choose normal coordinates $\left\{z^{1}, \ldots, z^{h^{2,1}}\right\}$ at $z_{0} \in \mathcal{M}$. We let $\Omega$ be a local normal frame for $H^{3,0} \rightarrow \mathcal{M}$ at $z_{0}$, and we let $\omega=d x+\tau d y$. Recall that $\omega$ is not a normal frame, since $\left|\omega_{\tau}\right|=(\operatorname{Im} \tau)^{1 / 2}$. We let $\widetilde{e}_{\mathcal{L}}=\left(\operatorname{Im} \tau_{0}\right)^{1 / 2} \Omega^{*} \otimes \omega^{*}$, so that $\left|\widetilde{e}_{\mathcal{L}}\left(Z_{0}\right)\right|=1$.

As in $\$ 3.2$ the matrix $\left(H_{j k}\right)$ of the holomorphic Hessian is given by

$$
H^{\prime}\left(Z_{0}\right)=\left.\sum_{j, q} H_{j q}^{\prime} d z^{q} \otimes d z^{j} \otimes \widetilde{e}_{\mathcal{L}}\right|_{Z_{0}}, \quad 0 \leq j, q \leq h^{2,1},
$$

where

$$
\left.d z^{0}\right|_{Z_{0}}=\left.\frac{1}{\operatorname{Im} \tau_{0}} d \tau\right|_{z_{0}}
$$

is the unit holomorphic cotangent vector (with respect to the Weil-Petersson, or hyperbolic, metric on $\mathcal{E}$ ) at $\tau_{0}$.

We wish to express formulas (59)-(60) for the complex Hessian in terms of these coordinates and frames. We write

$$
\left(\nabla_{j} f\right) \otimes e_{\mathcal{L}}=\nabla_{\partial / \partial z^{j}}\left(f e_{\mathcal{L}}\right), \quad 1 \leq j \leq h^{2,1}, \quad\left(\nabla_{0} f\right) \otimes e_{\mathcal{L}}=\left(\operatorname{Im} \tau_{0}\right) \nabla_{\partial / \partial \tau}\left(f e_{\mathcal{L}}\right) .
$$

( $\nabla_{0}$ is the normalized covariant $\tau$-derivative given by (87).) The complex Hessian matrix is given by:

$$
H^{c}\left(Z_{0}\right)=\left(\begin{array}{ll}
H^{\prime}\left(Z_{0}\right) & f\left(Z_{0}\right) I \\
\hline f\left(Z_{0}\right) I & \overline{H^{\prime}\left(Z_{0}\right)}
\end{array}\right), \quad H^{\prime}=\left(\nabla_{j} \nabla_{q} f\right)_{0 \leq j, q \leq h^{2,1}} .
$$

Identifying the off-diagonal components with $f\left(Z_{0}\right) \in \mathbb{C}$, we view the image space as a subspace of $\operatorname{Sym}\left(h^{2,1}+1, \mathbb{C}\right) \oplus \mathbb{C}$, so we can write the Hessian map in the form

$$
H_{Z_{0}}: \mathcal{S}_{Z} \rightarrow \operatorname{Sym}\left(h^{2,1}+1, \mathbb{C}\right) \oplus \mathbb{C}, \quad W \mapsto\left(H^{\prime}\left(Z_{0}\right), f\left(Z_{0}\right)\right) \text {. }
$$

Lemma 6.1. The range of the Hessian map $H_{Z_{0}}: \mathcal{S}_{Z_{0}} \rightarrow \operatorname{Sym}\left(h^{2,1}+1, \mathbb{C}\right) \oplus \mathbb{C}$ is of the form $\mathcal{H}_{Z_{0}} \oplus \mathbb{C}$, where $\mathcal{H}_{Z_{0}}$ is a real subspace of $\operatorname{Sym}\left(h^{2,1}+1, \mathbb{C}\right)$ of real dimension $2 h^{2,1}$ spanned over $\mathbb{R}$ by the matrices

$$
\xi^{k}=\left(\begin{array}{cc}
0 & e_{k} \\
e_{k}^{t} & \mathcal{F}^{k}(z)
\end{array}\right), \quad \xi^{h^{2,1}+k}=\left(\begin{array}{cc}
0 & \sqrt{-1} e_{k} \\
\sqrt{-1} e_{k}^{t} & -\sqrt{-1} \mathcal{F}^{k}(z)
\end{array}\right), \quad 1 \leq k \leq h^{2,1},
$$


given by (27), where $e_{k}$ is the $k$-th standard basis element of $\mathbb{C}^{h^{2,1}}$ and $\mathcal{F}^{k}(z) \in \operatorname{Sym}\left(h^{2,1}, \mathbb{C}\right)$ is the matrix $\left(\mathcal{F}_{j q}^{\bar{k}}(z)\right)$ of (46).

In other words, $\mathcal{H}_{Z_{0}}$ is the set of matrices of the form

$$
\left(\begin{array}{cc}
0 & \left(\bar{v}_{1}, \ldots, \bar{v}_{h^{2,1}}\right) \\
\left(\bar{v}_{1}, \ldots, \bar{v}_{h^{2,1}}\right)^{t} & \sum_{k=1}^{h^{2,1}} \mathcal{F}^{k}(z) v_{k}
\end{array}\right), \quad\left(v_{1}, \ldots, v_{h^{2,1}}\right) \in \mathbb{C}^{h^{2,1}} .
$$

We emphasize that $\mathcal{H}_{Z} \subset \operatorname{Sym}\left(h^{2,1}+1, \mathbb{C}\right)$ is only a real and not a complex subspace. We also note that $\operatorname{dim}_{\mathbb{R}} \mathcal{H}_{Z}=2 h^{2,1}$ and hence $\operatorname{dim}_{\mathbb{R}}\left(\mathcal{H}_{Z} \oplus \mathbb{C}\right)=b_{3}=\operatorname{dim}_{\mathbb{R}} \mathcal{S}_{Z}$; i.e., $H_{Z}$ is an isomorphism.

Proof of Lemma 6.1; We shall use the notation $1 \leq j, k, l \leq h^{2,1}, 0 \leq \alpha, \beta, \gamma \leq h^{2,1}$. By (74), we have the (real-linear) isomorphism

$$
\widetilde{\mathcal{W}}_{Z_{0}}=\mathcal{W} \circ I_{\tau}^{-1}: H_{z_{0}}^{2,1} \oplus H_{z_{0}}^{0,3} \stackrel{\approx}{\rightarrow} \mathcal{S}_{Z_{0}} \text {. }
$$

Recall that $H_{z_{0}}^{2,1} \oplus H_{z_{0}}^{0,3}$ has a complex orthonormal basis $\left\{\chi_{\alpha}\right\}$ of the form

$$
\chi_{j}=\mathcal{D}_{j} \Omega_{Z_{0}}, \quad 1 \leq j \leq h^{2,1}, \quad \chi_{0}=\bar{\Omega}_{Z_{0}} .
$$

By (76), a real orthonormal basis of $\mathcal{S}_{Z_{0}}$ is

$$
U_{\alpha}:=(\operatorname{Im} \tau)^{1 / 2} \widetilde{\mathcal{W}}_{Z_{0}}\left(\chi_{\alpha}\right), \quad V_{\alpha}:=(\operatorname{Im} \tau)^{1 / 2} \widetilde{\mathcal{W}}_{Z_{0}}\left(\sqrt{-1} \chi_{\alpha}\right)
$$

We write:

$$
U_{\alpha}=f_{\alpha} \widetilde{e}_{\mathcal{L}}, \quad V_{\alpha}=g_{\alpha} \widetilde{e}_{\mathcal{L}}
$$

equivalently

$$
\widetilde{\mathcal{W}}_{Z_{0}}\left(\chi_{\alpha}\right)=f_{\alpha} e_{\mathcal{L}}, \quad \widetilde{\mathcal{W}}_{Z_{0}}\left(\sqrt{-1} \chi_{\alpha}\right)=g_{\alpha} e_{\mathcal{L}}
$$

We must compute the matrices

$$
H_{Z_{0}}^{\prime}\left(f_{\alpha} \widetilde{e}_{\mathcal{L}}\right)=\left.\left(\nabla_{\beta} \nabla_{\gamma} f_{\alpha}\right)\right|_{Z_{0}}, \quad H_{Z_{0}}^{\prime}\left(g_{\alpha} \widetilde{e}_{\mathcal{L}}\right)=\left.\left(\nabla_{\beta} \nabla_{\gamma} g_{\alpha}\right)\right|_{Z_{0}},
$$

where $H_{Z_{0}}^{\prime}: \mathcal{S}_{Z_{0}} \rightarrow \operatorname{Sym}\left(h^{2,1}+1, \mathbb{C}\right)$ is the holomorphic Hessian map.

We shall show that:

$$
\left\{\begin{array}{l}
\text { (i) } \nabla_{0}^{2} f_{G}\left(Z_{0}\right)=0, \quad \forall G \in H_{Z_{0}}^{3}(X, \mathbb{C}) \quad\left(\text { where } W_{G}=f_{G} e_{\mathcal{L}}\right) \\
\text { and thus } \nabla_{0}^{2} f_{\alpha}\left(Z_{0}\right)=\nabla_{0}^{2} g_{\alpha}\left(Z_{0}\right)=0 \\
\text { (ii) } \nabla_{j} \nabla_{0} f_{0}\left(Z_{0}\right)=\nabla_{j} \nabla_{0} g_{0}\left(Z_{0}\right)=0, \\
\text { (iii) } \nabla_{k} \nabla_{j} f_{0}\left(Z_{0}\right)=\nabla_{k} \nabla_{j} g_{0}\left(Z_{0}\right)=0, \\
\text { (iv) } \nabla_{k} \nabla_{0} f_{j}\left(Z_{0}\right)=-\sqrt{-1} \delta_{j k}, \quad \nabla_{k} \nabla_{0} g_{j}\left(Z_{0}\right)=-\delta_{j k}, \\
\text { (v) } \nabla_{k} \nabla_{l} f_{j}\left(Z_{0}\right)=\mathcal{F}_{k l}^{\bar{j}}, \quad \nabla_{k} \nabla_{l} g_{j}\left(Z_{0}\right)=\sqrt{-1} \mathcal{F}_{k l}^{\bar{j}} .
\end{array}\right.
$$

First,

$$
\nabla_{0} f_{G}(z, \tau)=\frac{\left|\operatorname{Im} \tau_{0}\right|}{\operatorname{Im} \tau} \int_{X}(F+\bar{\tau} H) \wedge \Omega_{z}
$$


It follows that

$$
\nabla_{0}^{2} f_{G}\left(z_{0}, \tau_{0}\right)=\frac{\left|\operatorname{Im} \tau_{0}\right|^{2}}{\operatorname{Im} \tau} \frac{\partial}{\partial \tau} \int_{X}(F+\bar{\tau} H) \wedge \Omega_{z}=0
$$

by the critical point equation $\nabla_{0} f_{G}\left(z_{0}, \tau_{0}\right)=0$. This proves (i).

Next, differentiating (103) with $f_{G}=f_{\alpha}$, we get

$$
\nabla_{j} \nabla_{0} f_{\alpha}\left(Z_{0}\right)=\int \overline{\chi_{\alpha}} \wedge \mathcal{D}_{j} \Omega_{Z_{0}}=\int \overline{\chi_{\alpha}} \wedge \chi_{j}=-i \delta_{j \alpha}
$$

and similarly,

$$
\nabla_{j} \nabla_{0} g_{\alpha}\left(Z_{0}\right)=\int \overline{i \chi_{\alpha}} \wedge \chi_{j}=-\delta_{j \alpha}
$$

This verifies (ii) and (iv).

Finally, we have by (46),

$$
\nabla_{k} \nabla_{j} f_{\alpha}=\int \chi_{\alpha} \wedge \mathcal{D}_{k} \mathcal{D}_{j} \Omega=-i \sum_{l} \mathcal{F}_{k j}^{\bar{l}} \int \chi_{\alpha} \wedge \overline{\mathcal{D}_{l} \Omega}
$$

and hence

$$
\nabla_{k} \nabla_{j} f_{\alpha}\left(Z_{0}\right)=-i \sum_{l} \mathcal{F}_{k j}^{\bar{l}} \int \chi_{\alpha} \wedge \bar{\chi}_{l}=-i \sum_{l} \mathcal{F}_{k j}^{\bar{l}} \delta_{l \alpha}=\left\{\begin{array}{ll}
-i \mathcal{F}_{k j}^{\bar{\alpha}} & \text { for } \quad \alpha \geq 1 \\
0 & \text { for } \alpha=0
\end{array} .\right.
$$

We also have $\nabla_{k} \nabla_{j} g_{\alpha}\left(Z_{0}\right)=i \nabla_{k} \nabla_{j} f_{\alpha}\left(Z_{0}\right)$, verifying (iii) and (v).

Thus, the holomorphic Hessian $H^{\prime}\left(Z_{0}\right)$ maps the orthonormal fluxes

$$
i U_{1}, \ldots, i U_{h^{2,1}},-i V_{1}, \ldots,-i V_{h^{2,1}}
$$

to the matrices $\xi^{1}, \ldots, \xi^{2 h^{2,1}}$ given by (27). Furthermore,

$$
f_{0}\left(Z_{0}\right)=1, H^{\prime}\left(U_{0}\right)=0, g_{0}\left(Z_{0}\right)=i, H^{\prime}\left(V_{0}\right)=0,
$$

while

$$
f_{j}\left(Z_{0}\right)=g_{j}\left(Z_{0}\right)=0 .
$$

Thus $H^{c}\left(Z_{0}\right)$ maps the orthonormal fluxes (104) to the elements $\xi^{a} \oplus 0 \in \operatorname{Sym}\left(h^{2,1}+1, \mathbb{C}\right) \oplus \mathbb{C}$, and maps $U_{0}$ to $0 \oplus 1$ and $V_{0}$ to $0 \oplus i$.

6.2. Distortion of inner product under the Hessian map. We recall that the space $\operatorname{Sym}\left(h^{2,1}+1, \mathbb{C}\right)$ of complex symmetric matrices, regarded as a real vector space, has the inner product

$$
(A, B)_{\mathbb{R}}=\operatorname{Re}\langle A, B\rangle_{H S}=\operatorname{Re}\left(\text { Trace } A B^{*}\right) .
$$

Recalling that $\mathcal{S}_{Z}=\widetilde{\mathcal{W}}_{Z}\left(H_{z}^{2,1} \oplus H_{z}^{0,3}\right)$, we consider its codimension 1 subspace

$$
\mathcal{S}_{Z}^{\prime}=\widetilde{\mathcal{W}}_{Z}\left(H_{z}^{2,1}\right)
$$

By the proof of Lemma 6.1, the holomorphic Hessian map

$$
H_{Z}: \mathcal{S}_{Z}^{\prime} \rightarrow \mathcal{H}_{Z}
$$

is bijective, but as a map between inner product spaces, it is not an isometry. The distortion is given by the positive definite operator $\Lambda_{Z}$. We write

$$
\Lambda_{Z} \xi^{a}=\sum_{b=1}^{2 h^{2,1}} \Lambda_{a b} \xi^{b}
$$


so that

$$
\left(\xi^{a}, \xi^{b}\right)_{\mathbb{R}}=\left(\Lambda_{Z}^{-1} \Lambda_{Z} \xi^{a}, \xi^{b}\right)_{\mathbb{R}}=\sum_{c} \Lambda_{a c}\left(\Lambda_{Z}^{-1} \xi^{c}, \xi^{b}\right)_{\mathbb{R}}=\sum_{c} \Lambda_{a c} \delta_{c b}=\Lambda_{a b}
$$

Tracing through the definitions, we obtain that $\left(\Lambda_{a b}\right)$ is the matrix

$$
\left(\begin{array}{cc}
\Lambda^{\prime} & \Lambda^{\prime \prime} \\
\Lambda^{\prime \prime} & \Lambda^{\prime}
\end{array}\right), \quad \Lambda_{j k}^{\prime}=2 \delta_{j k}+\operatorname{Re} \operatorname{Tr} \mathcal{F}^{j} \mathcal{F}^{k *}, \quad \Lambda_{j k}^{\prime \prime}=\operatorname{Im} \operatorname{Tr} \mathcal{F}^{j} \mathcal{F}^{k *} .
$$

of Hilbert-Schmidt inner products of the matrices in Lemma 6.1. Hence,

$$
\Lambda_{j k}^{\prime}+\sqrt{-1} \Lambda_{j k}^{\prime \prime}=2 \delta_{j k}+\operatorname{Tr} \mathcal{F}^{j} \mathcal{F}^{k *}
$$

To tie this discussion together with that in [AD] and [DSZ2, §2.1], we note that we can consider $\mathcal{H}_{Z}$ as a complex vector space by redefining complex multiplication in $\mathcal{H}_{Z}$ :

$$
c \odot\left(\begin{array}{cc}
0 & u \\
u^{t} & A
\end{array}\right)=\left(\begin{array}{cc}
0 & \bar{c} u \\
\bar{c} u^{t} & c A
\end{array}\right) .
$$

We then define a Hermitian inner product on $\mathcal{H}_{Z}$ :

$$
\left(\left(\begin{array}{cc}
0 & u \\
u^{t} & A
\end{array}\right), \overline{\left(\begin{array}{cc}
0 & v \\
v^{t} & B
\end{array}\right)}\right)=2 \bar{u} \cdot v+\operatorname{Tr}\left(A B^{*}\right) .
$$

We recall from (29) that

$$
\Lambda_{Z}=\sum_{j=1}^{h^{2,1}} \xi^{j} \otimes \xi^{j *}
$$

where the $\xi^{j}$ are $\left(h^{2,1}+1\right) \times\left(h^{2,1}+1\right)$ matrices. Each term $\xi^{j} \otimes \xi^{j *}$ in $\Lambda_{Z}$ may be expressed in matrix form as $\left(\xi_{a b}^{j} \bar{\xi}_{c d}^{j}\right)$; i.e.,

$$
\left(\Lambda_{Z} H\right)_{k l}=\sum_{p, q}\left[\Lambda_{Z}\right]_{k l}^{p q} H_{p q}, \quad\left[\Lambda_{Z}\right]_{k l}^{p q}=\sum_{j=1}^{h^{2,1}} \xi_{k l}^{j} \bar{\xi}_{p q}^{j}, \quad 0 \leq k, l, p, q \leq h^{2,1} .
$$

As in [DSZ2, §2.1], the result may be expressed in terms of the Szegö kernel $\Pi_{Z}$, i.e. the kernel of the orthogonal projection onto $\mathcal{S}_{Z}$. By (102) and (109), we have

$$
\left[\Lambda_{Z}\right]_{k l}^{p q}=\left.\nabla_{\zeta_{k}} \nabla_{\zeta_{l}} \nabla_{\bar{\eta}_{p}} \nabla_{\bar{\eta}_{q}} F_{Z}(\zeta, \eta)\right|_{\zeta=\eta=Z}
$$

where $F_{Z}$ is the local representative of $\Pi_{Z}$ in a frame (cf. [DSZ2]).

In addition, $\Lambda_{Z}$ determines an operator $\tilde{\Lambda}_{Z}$ on the space $\mathcal{H}^{c}$ of complex matrices of the form

$$
H^{c}:=\left(\begin{array}{cc}
H & x I \\
\bar{x} I & \bar{H}
\end{array}\right), H \in \operatorname{Sym}\left(h^{21}, \mathbb{C}\right),
$$

defined by

$$
\tilde{\Lambda}_{Z}\left(\begin{array}{cc}
H & x I \\
\bar{x} I & \bar{H}
\end{array}\right)=\left(\begin{array}{cc}
\Lambda_{Z} H & x I \\
\bar{x} I & \overline{\Lambda_{Z} H}
\end{array}\right)
$$

We now relate the $(1,1)$-form $\omega_{\Lambda}$ of (31) and the operator $\Lambda$ to the curvature of the Weil-Petersson metric on $\mathcal{C}$. 
Proposition 6.2. We have:

i) $\left[\Lambda_{Z}\right]_{j^{\prime} q^{\prime}}^{j q}=-G^{q \bar{p}} R_{j^{\prime} q^{\prime} \bar{p}}^{j}+\delta_{j^{\prime}}^{j} \delta_{q^{\prime}}^{q}+\delta_{q^{\prime}}^{j} \delta_{j^{\prime}}^{q}$, where $R$ is the curvature tensor of the WeilPetersson metric on $\mathcal{C}$;

ii) $\omega_{\Lambda}=(m+3) \omega_{W P}+\operatorname{Ric}\left(\omega_{W P}\right)$ where Ric is the Ricci curvature $(1,1)$ form of the Weil-Petersson metric of $\mathcal{M}$, i.e.

$$
\operatorname{Ric}\left(\omega_{W P}\right)=\frac{i}{2} \sum_{i \bar{j}} \operatorname{Ric}_{i \bar{j}} d z^{i} \wedge d \bar{z}^{j}, \quad R i c_{i \bar{j}}:=-G^{k \bar{\ell}} R_{i \bar{j} \bar{k}} .
$$

Thus, $\omega_{\Lambda}$ is the Hodge metric [Lu, Wa2].

Proof. To prove (i), it suffices to combine (110) and (52), raising and lowering indices as appropriate. (In (110), a normal frame at $Z$ is assumed.)

For (ii) we note that the $(1,1)$-form

$$
\omega_{\Lambda}=\frac{i}{2} \sum\left[2 \delta_{i j}+\operatorname{Tr} \mathcal{F}^{i}(Z) \mathcal{F}^{j *}(Z)\right] d z^{i} \wedge d \bar{z}^{j}
$$

On the other hand, by (47),

$$
\begin{aligned}
R i c_{i \bar{j}} & =-G^{k \bar{\ell}}\left[G_{i \bar{j}} G_{k \bar{\ell}}+G_{i \bar{\ell}} G_{k \bar{j}}-\frac{1}{\int_{\mathcal{M}} \Omega \wedge \bar{\Omega}} \sum_{p, q} G^{p p \bar{q}} \mathcal{F}_{i k p} \overline{\mathcal{F}_{j \ell q}}\right] \\
& =-(m+1) G_{i \bar{j}}+\operatorname{Tr}_{\mathcal{F}} \mathcal{F}^{i} \mathcal{F}^{j *}
\end{aligned}
$$

Remark: To facilitate comparison with [AD, DSZ1, we note that our notational conventions are the same as in DSZ1]. In [AD, the Szegö kernel $\Pi_{Z}$ is denoted $G_{Z}$. The formulas in [AD] (4.8) are the same as (110), resp. Proposition 6.2(1). Also $F_{a b \mid \bar{c} \bar{d}}=\Lambda_{a b}^{p q} G_{p \bar{c}} G_{q \bar{d}}$. The coefficients $F_{a \bar{b} \mid c \bar{d}}$ in $[\mathrm{AD}]$ correspond to the off-diagonal blocks of $\tilde{\Lambda}$.

6.3. Proof of Theorem 1.8, All but one of the ingredients of the proof are precisely the same as in Theorem 1.4. We first define the analogue of (25) and (32) for the signed sum:

$$
\begin{aligned}
\operatorname{Ind}(Z) & :=\int_{\mathcal{S}_{Z}} \operatorname{det} H^{c} W(Z) \chi_{Q_{Z}} d W \\
& =\frac{1}{b_{3} ! \sqrt{\operatorname{det} \Lambda_{Z}}} \int_{\mathcal{H}_{Z} \oplus \mathbb{C}} \operatorname{det}\left(H^{*} H-|x|^{2} I\right) e^{-\left(\Lambda_{Z}^{-1} H, H\right)_{\mathbb{R}}-|x|^{2}} d H d x .
\end{aligned}
$$

By Lemma 4.2 and the proof of Lemma 4.3, we conclude that

$$
\mathcal{I}_{n d_{\chi_{K}}}(L)=L^{b_{3}}\left[\int_{K} \mathcal{I} n d(Z) d \operatorname{Vol}_{W P}+O\left(L^{-1 / 2}\right)\right] .
$$

To complete the proof of Theorem 1.8, we evaluate the integral in (116):

Lemma 6.3. We have

$$
b_{3} ! \mathcal{I} n d(Z) d \operatorname{Vol}_{W P}=\frac{\pi^{2 m}}{2^{m}} c_{m}\left(T^{*(1,0)}(\mathcal{C}) \otimes \mathcal{L}, \omega_{W P} \otimes h_{W P}^{*}\right)=\left(\frac{\pi}{2}\right)^{m} \operatorname{det}(-R-\omega \otimes I) .
$$


Proof. This follows by a supersymmetric formula for the determinant, used in this context in [AD] and also in [BSZ2]. We briefly review the fermionic formalism referring to [BGV], BSZ2] for further details in a similar setting.

Let $M=\left(M_{j^{\prime}}^{j}\right)$ be an $n \times n$ complex matrix. Then,

$$
\operatorname{det} M=\int^{B^{2 n}} e^{-\langle M \eta, \bar{\eta}\rangle} d \eta, \quad\langle M \eta, \bar{\eta}\rangle=\sum_{j, j^{\prime}} \eta_{j} M_{j^{\prime}}^{j} \bar{\eta}_{j^{\prime}}
$$

where $\eta_{j}, \bar{\eta}_{j}(1 \leq j \leq n)$ are anti-commuting (or "fermionic") variables. The integral $\int^{B}=$ $\int^{B^{2 n}}$ is the Berezin integral, a notation for the linear functional $\int^{B}: \bigwedge^{\bullet} \mathbb{C}^{2 n} \rightarrow \mathbb{C}$ defined by

$$
\int^{B} \mid \wedge^{t} \mathbb{C}^{2 n}=0 \quad \text { for } t<2 n, \quad \int^{B}\left(\prod_{j} \bar{\eta}_{j} \eta_{j}\right)=1
$$

We now apply this formalism to $\operatorname{det}\left(H^{*} H-|x|^{2} I\right)=\operatorname{det} H^{c}$ where $H^{c}$ is defined as in (112) and refer to the discussion in $\$ 6.2$. The matrix $H^{c}$ is of rank $b_{3}$, and we write

$$
\operatorname{det} H^{c}=\int^{B^{2 b_{3}}} e^{-\left\langle H^{c}(\eta, \bar{\eta}),(\theta, \bar{\theta})\right\rangle} d \eta d \theta
$$

where $\eta=\left(\eta_{1}, \ldots, \eta_{b_{3} / 2}\right), \theta=\left(\theta_{1}, \ldots, \theta_{b_{3} / 2}\right)$, and

$$
\left\langle H^{c}(\eta, \bar{\eta}),(\theta, \bar{\theta})\right\rangle=\sum\left(H_{j k} \eta_{j} \theta_{k}+x \delta_{j k} \eta_{j} \bar{\theta}_{k}+\bar{x} \delta_{j k} \bar{\eta}_{j} \theta_{k}+\bar{H}_{j k} \bar{\eta}_{j} \bar{\theta}_{k}\right)
$$

The quadratic form $\left(\Lambda_{Z}^{-1} H, H\right)_{\mathbb{R}}+|x|^{2}$ in the exponent of the Gaussian integral may be expressed in the form $\frac{1}{2}\left(\tilde{\Lambda}_{Z}^{-1} H^{c}, H^{c}\right)$, where $\tilde{\Lambda}_{Z}$ is the restriction of the operator defined in (113) to $\mathcal{H}_{Z}^{c}$. Indeed, both quadratic forms are equivalent to $Q_{Z}(W, W)$ under a linear change of variables $\left(W \rightarrow H_{Z}(W)\right.$ in the case of $\Lambda_{Z}$ and $W \rightarrow H^{c}(W)$ in the case of $\left.\tilde{\Lambda}_{Z}\right)$.

Then

$$
b_{3} ! \mathcal{I} n d(Z)=\frac{1}{\sqrt{\operatorname{det} \tilde{\Lambda}_{Z}}} \int_{\mathcal{H}_{Z}^{c}} \int^{B^{2 b_{3}}} e^{-\left\langle H^{c}(\eta, \bar{\eta}),(\theta, \bar{\theta})\right\rangle-\left\langle\tilde{\Lambda}_{Z}^{-1} H^{c}, H^{c}\right\rangle} d H^{c} d \eta d \theta .
$$

We let

$$
\Omega=(\eta, \bar{\eta}) \otimes(\theta, \bar{\theta})^{t}=\left(\begin{array}{cc}
\left(\eta_{j} \theta_{k}\right) & \left(\eta_{j} \bar{\theta}_{k}\right) \\
\left(\bar{\eta}_{j} \theta_{k}\right) & \left(\bar{\eta}_{j} \bar{\theta}_{k}\right)
\end{array}\right),
$$

so that $\left\langle H^{c}(\eta, \bar{\eta}),(\theta, \bar{\theta})\right\rangle=\left(H^{c}, \Omega\right)=\operatorname{Tr} H^{c} \Omega^{t}$. Then the $d H^{c}$ integral in (120) becomes the Fourier transform of the Gaussian function $e^{-\left\langle\tilde{\Lambda}^{-1} H^{c}, H^{c}\right\rangle}$ evaluated at $i \Omega$. Recalling that the Fourier transform of $e^{-\langle A x, x\rangle / 2}$ equals $(2 \pi)^{n / 2}(\operatorname{det} A)^{-1 / 2} e^{-\left\langle A^{-1} \xi, \xi\right\rangle / 2}$, we have that the $d H^{c}$ integral equals $(\operatorname{det} \tilde{\Lambda})^{\frac{1}{2}} e^{-\frac{1}{4}\langle\tilde{\Lambda} \Omega, \Omega\rangle}$. After cancelling $(\operatorname{det} \tilde{\Lambda})^{\frac{1}{2}}$, we obtain

$$
b_{3} ! \mathcal{I} n d(Z)=\pi^{m} \int^{B^{2 b_{3}}} e^{-\frac{1}{4}(\tilde{\Lambda} \Omega, \Omega)_{\mathbb{R}}} d \eta d \theta
$$


where in normal coordinates, we have (by (113) and Proposition 6.2)

$$
\begin{aligned}
\left(\tilde{\Lambda}_{Z} \Omega, \Omega\right)_{\mathbb{R}} & =\operatorname{Trace}\left[\left(\begin{array}{cc}
\Lambda_{Z} \eta \otimes \theta & \eta \otimes \bar{\theta} \\
\bar{\eta} \otimes \theta & \bar{\Lambda}_{Z} \bar{\eta} \otimes \bar{\theta}
\end{array}\right)\left(\begin{array}{cc}
\eta \otimes \theta & \eta \otimes \bar{\theta} \\
\bar{\eta} \otimes \theta & \bar{\eta} \otimes \bar{\theta}
\end{array}\right)^{*}\right] \\
& =\sum_{j q j^{\prime} q^{\prime}}\left(\Lambda_{j^{\prime} q^{\prime}}^{j q} \eta_{j} \theta_{q} \bar{\eta}_{j^{\prime}} \overline{\theta_{q^{\prime}}}+\bar{\Lambda}_{j^{\prime} q^{\prime}}^{j q} \bar{\eta}_{j} \bar{\theta}_{q} \eta_{j^{\prime}} \theta_{q^{\prime}}\right)+\sum_{j q}\left(\eta_{j} \bar{\theta}_{q} \bar{\eta}_{j} \theta_{q}+\bar{\eta}_{j} \theta_{q} \eta_{j} \bar{\theta}_{q}\right) \\
& =2 \sum_{j q j^{\prime} q^{\prime}}\left(\Lambda_{j^{\prime} q^{\prime}}^{j q}-\delta_{j j^{\prime}} \delta_{q q^{\prime}}\right) \eta_{j} \theta_{q} \bar{\eta}_{j^{\prime}} \bar{\theta}_{q^{\prime}} \\
& =2 \sum_{j q j^{\prime} q^{\prime}}\left(R_{j \bar{j}^{\prime} q \bar{q}^{\prime}}+\delta_{j q} \delta_{j^{\prime} q^{\prime}}\right) \eta_{j} \theta_{q} \bar{\eta}_{j^{\prime}} \overline{\theta_{q^{\prime}}} .
\end{aligned}
$$

(Here we used the fact that $\bar{\Lambda}_{j^{\prime} q^{\prime}}^{j q}=\Lambda_{j q}^{j^{\prime} q^{\prime}}$; see (110).) Thus

$$
\begin{aligned}
b_{3} ! \mathcal{I} n d(Z) & =\pi^{m} \int^{B^{2 b_{3}}} e^{-\frac{1}{2}\left(R_{j j^{\prime} q q^{\prime}}+\delta_{j q^{\prime}} \delta_{j^{\prime} q^{\prime}}\right) \eta_{j} \bar{\eta}_{j^{\prime}} \theta_{q} \bar{\theta}_{q^{\prime}}} d \eta d \theta \\
& =\left(\frac{\pi}{2}\right)^{m} \frac{\operatorname{det}(-R-\omega \otimes I)}{d \mathrm{Vol}_{W P}}
\end{aligned}
$$

Remark: The index density computation in special geometry is closely related to the asymptotics in [DSZ2, §5] for critical point densities for powers of a positive line bundle $L$ on a compact Kähler manifold $M$. The expansions in $\S 5.1$ of [DSZ2] can be used to show that the (first few) terms in the asymptotic expansion of the index density equal those of the Chern form corresponding to $c_{m}\left(T^{* 1,0} \otimes L^{N}\right)$.

6.4. Examples. We describe in this section the critical point distribution for the cases where the dimension $h^{2,1}(X)$ of the moduli space is 0 and 1 , i.e. when $\operatorname{dim} \mathcal{C}$ is 1 and 2 , respectively.

6.4.1. $h^{2,1}(X)=0$. The simplest example is the case where the Calabi-Yau manifold $X$ is rigid, i.e. $\mathcal{M}=\{p t\}$. (See [AD, DD1] for further details and computer graphics of critical points in this case.) Then only the parameter $\tau \in \mathcal{H}$ varies. Let $G=F+i H$, and consider the flux superpotential $W_{G}$. Its critical point equation is

$$
F+\tau H \in H^{0,3}
$$

(since in this case $H^{2,1}(X, \mathbb{C})=0$ ). So we write

$$
F=A \Omega+\overline{A \Omega}, \quad H=B \Omega+\overline{B \Omega}, \quad A=a_{1}+i a_{2}, \quad B=b_{1}+i b_{2} \in \mathbb{Z}+\sqrt{-1} \mathbb{Z} .
$$

Then writing $W_{G}=W_{A, B}$, we have

$$
\nabla W_{A, B}=0 \Longleftrightarrow F+\tau H \in H^{0,3} \Longleftrightarrow A+\tau B=0 \Longleftrightarrow \tau=-\frac{A}{B} .
$$

Each flux superpotential $W_{A, B} \in \mathcal{S}$ (with $A, B \in \mathbb{C}$ ) has a unique critical point in $\mathcal{H}$, which may or may not lie in the fundamental domain $\mathcal{C}$. In the notation of (15),

$$
\pi(\mathcal{S})=\left\{W_{A, B}:-\frac{A}{B} \in \mathcal{C}\right\}
$$


is a domain with boundary in $\mathbb{C}^{2}$. Each $S L(2, \mathbb{Z})$-orbit of fluxes (or superpotentials) contains a unique element whose critical point lies in $\mathcal{C}$, so $\pi(S)$ is a fundamental domain for the action of $\Gamma$ on $\mathcal{S}$.

Thus, counting critical points is equivalent to counting $S L(2, \mathbb{Z})$ orbits of superpotentials satisfying the tadpole constraint. The pair $(A, B)$ corresponds to the element $\left(\begin{array}{ll}a_{1} & b_{1} \\ a_{2} & b_{2}\end{array}\right) \in$ $G L(2, \mathbb{Z})$ and the Hodge-Riemann form quadratic form may be identified with the indefinite quadratic form

$$
Q[(A, B)]=a_{1} b_{2}-b_{2} a_{1}
$$

on $\mathbb{R}^{4}$. The modular group $S L(2, \mathbb{Z})$ acts by the standard diagonal action on $(A, B) \in \mathbb{R}^{2} \times \mathbb{R}^{2}$ preserving $Q[(A, B)]$ or equivalently by left multiplication preserving det. Thus, the set of superpotentials satisfying the tadpole constraint is parametrized by:

$$
\left\{\left(\begin{array}{ll}
a_{1} & b_{1} \\
a_{2} & b_{2}
\end{array}\right) \in G L(2, \mathbb{Z}): 0<\operatorname{det}\left(\begin{array}{ll}
a_{1} & b_{1} \\
a_{2} & b_{2}
\end{array}\right) \leq L\right\}
$$

and we want to count the number of $S L(2, \mathbb{Z})$-orbits in this set. Counting the number of $S L(2, \mathbb{Z})$ orbits in $\mathcal{D}_{L}$ is equivalent to determining the average order of the classical divisor function $\sigma(m)$, see for instance Hardy-Wright [HW], Theorem 324]:

$$
\mathcal{N}^{\text {crit }}(L)=\sum_{m=1}^{L} \sum_{k \mid m} k=\sum_{m=1}^{L} \sigma(m) \sim \frac{\pi^{2}}{12} L^{2}+O(L \log L) .
$$

As verified in [DD1] (and as follows very simply from Theorem [1.4), the critical points are uniformly distributed relative to the hyperbolic area form.

6.4.2. $h^{2,1}(X)=1$. We now illustrate our notation and results with the case where the moduli space of complex structures on $X$ is one-dimensional over $\mathbb{C}$. (This case is also studied in DD1 from a slightly different point of view.) In this case, there is a single Yukawa coupling $\mathcal{F}_{11}^{\overline{1}}(z)$ defined by $D_{z}^{2} \Omega_{z}=\mathcal{F}_{11}^{\overline{1}}(z) \overline{D_{z} \Omega_{z}}$.

The space $\mathcal{S}_{z, \tau} \simeq H^{2,1} \oplus H^{0,3} \simeq \mathbb{C}^{2}$. The space is spanned as a real vector space by four superpotentials $U_{0}, U_{1}, V_{0}, V_{1}$ corresponding to $\left\{\overline{\Omega_{z}}, \mathcal{D}_{z} \Omega_{z}, i \overline{\Omega_{z}}, i \mathcal{D}_{z} \Omega_{z}\right\}$. By the proof of Lemma 6.1, the holomorphic Hessians of $U_{0}$ and $V_{0}$ at a critical point equal zero, so we only need to consider the holomorphic Hessian map on $U_{1}$ and $V_{1}$. The corresponding space of Hessians is the real 2-dimensional subspace $\mathcal{H}_{Z}$ of $\operatorname{Sym}(2, \mathbb{C})$ spanned by

$$
\xi^{1}=\left(\begin{array}{cc}
0 & 1 \\
1 & F(z)
\end{array}\right), \quad \xi^{2}=\sqrt{-1}\left(\begin{array}{cc}
0 & 1 \\
1 & -F(z)
\end{array}\right)
$$

where we write $F=\mathcal{F}_{11}^{\overline{1}}$. Hence, we may parameterize the space $\mathcal{H}_{Z}$ of holomorphic Hessians by

$$
w=y_{1}+i y_{2} \mapsto H(w)=\left(\begin{array}{cc}
0 & w \\
w & F(z) \bar{w}
\end{array}\right)
$$

By (25), we have:

$$
\mathcal{K}^{\mathrm{crit}}(Z)=\frac{1}{2 !} \int_{\mathbb{C} \oplus \mathbb{C}}\left|\operatorname{det}\left(H(w)^{*} H(w)-|x|^{2} I\right)\right| e^{-|w|^{2}+|x|^{2}} d w d x .
$$


We note that

$$
\operatorname{det}\left(H(w)^{*} H(w)-|x|^{2} I\right)=|w|^{4}+|x|^{4}-\left(2+|F(z)|^{2}\right)|x|^{2}|w|^{2} .
$$

Hence

$$
\mathcal{K}^{\mathrm{crit}}(Z)=\left.\frac{1}{2 !} \int_{\mathbb{C} \oplus \mathbb{C}}|| w\right|^{4}+|x|^{4}-\left(2+|F(z)|^{2}\right)|x|^{2}|w|^{2} \mid e^{-|w|^{2}+|x|^{2}} d w d x,
$$

agreeing with (3.19) of [DD1]. There, the integral is evaluated as

$$
\mathcal{K}^{\mathrm{crit}}(Z)=\frac{\pi^{2}}{2}\left(2-|F|^{2}+\frac{2|F|^{3}}{\sqrt{4+|\tilde{F}|^{2}}}\right) \text {. }
$$

Remark: In this example, the discriminant variety is given by

$$
\widetilde{\mathcal{D}}=\left\{\left(Z, x W_{0}(Z)+w W_{1}(Z)\right) \in \mathcal{I}:|w|^{2}-|x|^{2}= \pm\left|w x F(z)^{2}\right|\right\},
$$

where $W_{\alpha}=U_{\alpha}+i V_{\alpha}$. The matrix $\Lambda$ is given by

$$
\Lambda=\left(\begin{array}{cc}
2+|F|^{2} & 0 \\
0 & 2+|F|^{2}
\end{array}\right) .
$$

\section{Problems And heuristics on the StRing Theory LANDSCAPE}

In this section, we continue the discussion begun in \$1.6 on the bearing of our methods and results on the physicists' picture of the string theory landscape. We briefly review some of the heuristic estimates in the physics discussions, and then discuss a number of mathematical pitfalls in the heuristics. In \$7.2, we state some mathematical problems suggested by the heuristics and by rigorous vacuum statistics. In \$7.3, we give our own (tentative) heuristic estimate of the dependence of the critical point density $\mathcal{K}^{\text {crit }}(Z)$ on the dimension $b_{3} / 2$ of $\mathcal{C}$.

7.1. Complexity of the string theory landscape. As mentioned in \$1.6 the possible vacua in string/M theory are often represented as valleys in a complex string theory landscape, and the number of valleys is often estimated at $10^{500}$.

L. Susskind and others have argued that such a large number of possible vacua should essentially be a consequence of the large number of variables in the potential. A common and general argument to arrive at this number of vacua without specifying any particular string theory model is to reason that the potential energy is a function of roughly 1000 variables. A generic polynomial $f$ of degree $d$ on $\mathbb{C}^{m}$ has $(d-1)^{m}$ critical points since critical points are solutions of the $m$ equations $\frac{\partial f}{\partial z_{j}}(w)=0$ of degree $d-1$. Thus, the number of critical points would seem to grow at an exponential rate in the number of variables. Such an exponential growth rate of critical points also appears in the physics of spin glasses, where the growth in the number of metastable states (local minima of the Hamiltonian) in terms of the number of variables is often used to measure the complexity of the energy landscape. In special model of random Hamiltonians on domains in $\mathbb{R}^{N}$, exponential growth of the number of local minima in $N$ has recently been proved rigorously $[\mathrm{Fy}$.

In the specific models of type IIb flux compactifications on a CY 3 -fold $X$, the number of variables is $b_{3}(X)$. As mentioned above, for a typical $C Y$-fold, $b_{3}$ is often around 300 and 
sometimes as high as 1000 (cf. GHJ, CO ), and therefore the scalar potential $V_{W}$ in (11) is a function of this number of variables. By naive counting of variables one would thus arrive at a figure like $10^{500}$ for such models. The more sophisticated estimate $N_{v a c} \simeq \frac{L^{b_{3}}}{b_{3} !} f\left(b_{3}\right)$ in flux compactifications (see \$1.6 for the notation) does not supplant the naive counting argument since the order of magnitude of $f\left(b_{3}\right)$ is unknown. We recall that it is the integral over $\mathcal{C}$ of the Gaussian integral in (32) (see (125). The Gaussian integral for $\mathcal{K}^{\text {crit }}$ in that line resembles to some extent the integral formula for the expected number of critical points in spin glass theory, which has exponential growth (see e.g. $[\mathrm{Fy}]$ ).

Although the naive counting of variables or the analogy to complexity of energy landscapes bring some insight into vacuum counting, we now point out some pitfalls in estimating numbers of vacua or the coefficient $f\left(b_{3}\right)$ in flux compactifications on this basis.

(1) The critical point equation (12) is $C^{\infty}$ but not holomorphic, so vacua are critical points of a real system of equations, and it is not obvious how many connection critical points to expect even a polynomial of a given degree to have. This number depends on the connection, and is studied in detail in [DSZ1, DSZ2] and in the present paper.

(2) A flux superpotential $W$ is not a polynomial and it is not clear how to assign it a 'degree' which reflects its number of critical points on all of Teichmüller space, or equivalently, the number of critical points in $\mathcal{C}$ corresponding to the $\Gamma$-orbit of $W$. Examples (e.g. in 6.4.1) show that this number can be relatively small.

(3) It seems reasonable to say that the number of fluxes rather than the number of critical points per flux that dominates the number of vacua. In flux compactifications, the landscape should therefore be viewed as the graph of the scalar potential $V_{W}(Z)$ on $\mathcal{C} \times \mathcal{S}$, i.e. as a function of both variables $W, Z$, and the local minima should be viewed as pairs $\left(W_{G}, Z\right)$ with $G \in H^{3}(X, \mathbb{Z} \oplus \sqrt{-1} \mathbb{Z})$ and with $Z \in \operatorname{Crit}\left(W_{G}\right)$.

(4) However (see the problems below) it is not straightforward

to define 'per vacua', since the tadpole constraint is hyperbolic, and the total number of lattice points in the shell $0<Q[G]<L$ is infinite.

(5) In estimating $\mathcal{K}^{\text {crit }}(Z)$ we are fixing $Z$ in the interior of $\mathcal{C}$. But there could exist singular points of $\mathcal{C}$ at which $\mathcal{K}^{\text {crit }}(Z)$ blows up (see DD1 for discussion of conifold points). It would also be interesting to study $\mathcal{K}^{\text {crit }}(Z)$ as $Z \rightarrow \partial \mathcal{C}$.

(6) As mentioned in \$1.6 (see also 97.3), there may be a significant difference between the order of magnitude of the density of critical points and of the number of critical points, since $\mathcal{C}$ is an incomplete Kähler manifold of possibly quite small volume. See [LS1 for the current state of the art on the volume. There is no analogue of the small volume of the configuration space in spin glass complexity.

(7) The tadpole constraint (11) becomes much more highly constraining as the number $b_{3}$ of variables increases for fixed $L$ and is responsible for the factor $\frac{1}{\left(b_{3}\right) !}$ in Theorem 1.4. Again, no such feature exists in complexity estimates in spin glasses.

7.2. Problems. The issues mentioned above (and the detailed heuristics in \$7.3) suggest a number of problems. The ultimate goal is:

Problem 7.1. Does string theory contain a vacuum consistent with the standard model, and if so, how many? Find examples of Calabi-Yau manifolds, and any other postulated structures, for which it is certain that such a vacuum exists. 
Now testing consistency with the standard model requires elucidating far more structure of a candidate vacuum - the gauge group, the matter content, and so forth - than we are considering here. To address this ultimate problem, one would need many more statistical results, along the lines set out in [Do. However one can make arguments (admittedly quite speculative at this point) that the dominant multiplicity in vacuum counting arises from the multiplicity of flux vacua we are discussing here. An important problem in this context is

Problem 7.2. How large does $L$ need to be to ensure that there exists a vacuum with

$$
\left|W_{G}(Z)\right|^{2} \leq \lambda_{*}
$$

for a specified $\lambda_{*}$ ? In that case, how many such vacua are there? Find examples of CalabiYau manifolds where it is certain that such a vacuum exists.

To solve this problem for type IIb flux compactifications, we would need to sharpen Theorem 1.4 in many ways which lead to the subsequent problems stated below.

The constraint (123) on $\left|W_{G}(Z)\right|^{2}$ is a simple example of 'consistency with the standard model.' If the real world were (counter-factually) exactly supersymmetric, this would be the constraint that the vacuum should have a cosmological constant $V_{W}(Z)=-3\left|W_{G}(Z)\right|^{2}$ (as in (11)) consistent with the known value. While the physical discussion requires taking supersymmetry breaking into account, as discussed in DD2, vacua can exist in which supersymmetry is broken by effects not taken into account here, making additional contributions to the vacuum energy which lift the exact vacuum energy to be consistent with the known value (essentially, zero). For such a vacuum, the quantity $3\left|W_{G}(Z)\right|^{2}$ would be the mass squared of the gravitino, a quantity which could be constrained by physical observations.

An independent motivation for (123) is that some proposals for stabilizing the moduli we did not discuss, such as that of [KKLT, are believed only to work under such a constraint.

In any case, as discussed in [DD1] (§3.3), one can count such vacua by choosing the test function to be $\theta\left(\lambda_{*}-\left|W_{G}(Z)\right|^{2}\right)$ where $\theta(x)=1$ for $x>0$ and $=0$ for $x \leq 0$. This test function is not homogeneous but can be handled by the methods of this paper (loc. cit.).

Theorem 1.4 is asymptotic in $L$ and we have also analyzed to some degree the $b_{3}$ dependence. But as mentioned in \$1.6, $L$ depends on the topology of $X$. There, we stated that in many examples $L \simeq C b_{3}$ with $1 / 3 \leq C \leq 3$. To bridge one gap between Theorem 1.4 and Problem 7.2, we state:

Problem 7.3. How are the order of magnitudes of $b_{3}(X)$ and $L$ of (36) related as $X$ varies over topologically distinct Calabi-Yau manifolds?

We have already mentioned the importance of obtaining effective estimates in $b_{3}$ of the coefficient (24) in Theorem 1.4.

Problem 7.4. Obtain an effective estimate of $\mathcal{K}^{\mathrm{crit}}(Z)$ and of its integral over $\mathcal{C}$ in $b_{3}$. Also, obtain such an estimate of the remainder.

Among the difficulties with this problem is that $\mathcal{K}^{\text {crit }}(Z)$ depends on special features of the moduli space $\mathcal{C}$ which depend on more than just the dimension $b_{3}$ and which may change in an irregular way as the dimension increases. We consider this problem below in $\$ 7.3$,

To gain insight into the size of the leading coefficient (24), one could write the principal term in Theorem 1.4 in the form $\frac{L^{b_{3}}}{b_{3} !} \times f\left(b_{3}\right)$ that is often used in string theory (cf. \$1.6), 
with $f\left(b_{3}\right)$ the Gaussian integral in (32). As mentioned above, it is natural to try to separate out the effects of the number of fluxes and the number of vacua per flux, or more precisely:

(1) the number of fluxes $G$ satisfying the tadpole constraint with a critical point in a compact subset $\mathcal{K} \subset \mathcal{C}$;

(2) the number of critical points 'per flux', or more precisely per $\Gamma$-orbit of fluxes, in $\mathcal{K}$ (see 6.4 .1 to clarify this distinction);

(3) the total number of critical points in $\mathcal{K}$ of all fluxes satisfying the tadpole constraint.

We can define the first quantity precisely as the sum

$$
\Theta_{K}(L)=\sum_{G \in H^{3}(X, \mathbb{Z} \oplus i \mathbb{Z}): Q[G] \leq L} \theta\left(\sum_{Z \in \mathcal{C}: \nabla W_{G}(Z)=0} \chi_{K}(Z)\right) .
$$

Thus, the problem we pose is:

Problem 7.5. Determine the asymptotics of $\Theta_{K}(L)$ as $L \rightarrow \infty$.

The second quantity is the ratio $\mathcal{N}_{K}(L) / \Theta_{K}(L)$. A possibly more tractable way to restate this problem is in terms of the 'average number of critical points' of a superpotential $W_{G}$ in $\mathcal{K}$. To define 'average' we need to introduce a probability measure on $\mathcal{F}$ which is compatible with $\chi_{Q} d W$. The most natural probability measures seem to be the normalized Gaussian measures $\gamma_{Z_{0}}$ on the spaces $\mathcal{S}_{Z_{0}}$ defined by the inner product $Q_{Z_{0}}$. Thus, we ask for the average number of critical points of $W \in \mathcal{S}_{Z_{0}}$ with respect to $\gamma_{Z_{0}}$. It would be interesting to study the number of critical points in a fixed $\mathcal{K} \subset \mathcal{C}$ or in all of $\mathcal{C}$ or indeed in all of Teichmüller space (which corresponds to counting critical points in $\mathcal{C}$ for a $\Gamma$-orbit of fluxes).

We observe that $W \in \mathcal{S}_{Z_{0}}$ has a critical point at $Z$ if and only if $W \in \mathcal{S}_{Z_{0}} \cap \mathcal{S}_{Z}$. In the case of flux superpotentials, $\operatorname{dim} \mathcal{S}_{Z_{0}}=\frac{1}{2} \operatorname{dim} \mathcal{F}$ so for generic pairs $Z, Z_{0}, \mathcal{S}_{Z_{0}} \cap \mathcal{S}_{Z}=\{0\}$. Thus, $\mathbf{E}_{Z_{0}}(\#$ Crits $(W))$ will be an integral over the special variety $\Sigma_{Z_{0}}=\left\{Z \in \mathbb{C}: \operatorname{dim} \mathcal{S}_{Z_{0}} \cap \mathcal{S}_{Z}>\right.$ $0\}$. This variety is obviously stratified by $h^{2,1}$ strata $\Sigma_{d}$ on which the dimension $d$ takes the values $d=1,2, \ldots, h^{2,1}$, and $\mathbf{E}_{Z_{0}}(\# \operatorname{Crits}(W))$ is a sum of integrals over each strata.

Problem 7.6. Determine the asymptotics of $\mathbf{E}_{Z_{0}}\left(\chi_{Q_{Z_{0}}(G / L)} \# \operatorname{Crits}\left(W_{G}\right)\right)$

We also recall that in Theorem [1.4 we ignored the effect of the discriminant variety and the boundary of the region of $\mathcal{C}$.

Problem 7.7. Estimate the remainder if $\psi$ does not vanish near the discriminant variety $\mathcal{D}$, or if $\psi$ is a characteristic function of a smooth region $K \subset \mathcal{C}$. Investigate the boundary behavior as $\mathcal{K}$ fills out to $\mathcal{C}$.

An analogue problem about studying accumulation of lattice points around boundaries of domains on non-degenerate surfaces is studied in [Ze1.

7.3. Heuristic estimate of the critical point density. We now present a heuristic estimate on the $b_{3}$-dependence of the critical point density (relative to the Weil-Petersson volume form)

$$
\mathcal{K}^{\text {crit }}(Z)=\left.\frac{1}{b_{3} ! \sqrt{\operatorname{det} \Lambda_{Z}}} \int_{\mathcal{H}_{Z} \oplus \mathbb{C}}\left|\operatorname{det} H^{*} H-\right| x\right|^{2} I \mid e^{-\left(\Lambda_{Z}^{-1} H, H\right)_{\mathbb{R}}-|x|^{2}} d H d x
$$


for $Z$ in regions of moduli space where the norm of $\Lambda_{Z}$ satisfies bounds independent of $b_{3}$. We recall (cf. Proposition [6.2) that $\Lambda_{Z}$ is the Hodge metric, hence we are studying the density of critical points in regions $K \subset \mathcal{C}$ where the absolute values of the eigenvalues of the Ricci curvature of the Weil-Petersson metric $\omega_{W P}$ are bounded by a uniform constant. In the notation $N_{v a c}(L) \sim \frac{L^{b_{3}}}{b_{3} !} f\left(b_{3}\right)$, we have

$$
f\left(b_{3}\right)=\left.\int_{\mathcal{C}} \chi_{K}(Z) \frac{1}{\sqrt{\operatorname{det} \Lambda_{Z}}} \int_{\mathcal{H}_{Z} \oplus \mathbb{C}}\left|\operatorname{det} H^{*} H-\right| x\right|^{2} I \mid e^{-\left(\Lambda_{Z}^{-1} H, H\right)_{\mathbb{R}}-|x|^{2}} d H d x
$$

where $\mathcal{K}$ is the region in which we are counting the critical points.

Our heuristic estimate is that the Gaussian integral (i.e. $b_{3} ! \mathcal{K}^{\text {crit }}(Z)$ ) has growth rate $\left(b_{3} / 2\right) ! N_{\mu}^{b_{3}}$ for $Z$ in a region $K=K_{\mu}$ of moduli space where $\left\|\Lambda_{Z}\right\| \leq \mu$. Here, $N_{\mu}$ is a constant depending only on $\mu$. It follows that $\mathcal{K}^{\text {crit }}(Z)$ would have the decay rate $b_{3}^{-b_{3} / 2}$ for $Z$ in $K_{\mu}$. We note that this heuristic estimate is consistent with the heuristic estimate given by Ashok-Douglas $\mathrm{AD}$ that $\mathcal{K}^{\text {crit }}(Z)$ should have the same order of magnitude as $\mathcal{I} n d(Z)$ (116). By Proposition 6.3, $b_{3} ! \mathcal{I} n d(Z)$ is a differential form depending polynomially on the curvature. The density of $b_{3} ! \mathcal{I} n d(Z)$ relative to $d V o l_{W P}=\frac{\omega_{W P}^{b_{3} / 2}}{\left(b_{3} / 2\right) !}$ thus has the growth $\left(b_{3} / 2\right) ! N_{\mu}^{b_{3}}$ we predict. We present the new heuristic to give evidence that the absolute value only changes the coefficient and not the order of magnitude in vacuum counting.

Before going into the heuristic estimate, we first discuss the consequences for vacuum counting. As mentioned in the introduction, it has been tentatively conjectured at this time of writing (Z. Lu) that the Weil-Petersson volume of $K_{\mu}$ is bounded above by the volume of a ball of radius $r(\mu)$ in $\mathbb{C}^{b_{3} / 2}$ depending only on $\mu$, and the latter volume decays like $\frac{1}{\left(b_{3} / 2\right) !}$. Thus it would appear that $N_{v a c, K_{\mu}}(L) \sim \frac{\left(C_{1} L N_{\mu}\right)^{b_{3}}}{b_{3} !}$. We include a constant $C_{1}$ to take into account the dependence on various parameters including $r(\mu)$, factors of $\pi$ and so on. If we then take the (often) observed value $L \sim C b_{3}$ with $C \in\left[\frac{1}{3}, 3\right]$, then the number of vacua in $K_{\mu}$ satisfying the tadpole constraint would grow at an exponential rate in $b_{3}$.

We now explain the heuristic estimate regarding the order of magnitude of $\mathcal{K}^{\text {crit }}(Z)(24)$ : the latter depends on two inputs, the subspace $\mathcal{H}_{Z}$ (or equivalently the orthogonal projection $P_{Z}$ onto $\left.\mathcal{H}_{Z}\right)$ and the eigenvalues of $\Lambda_{Z}$. To obtain upper and lower bounds on $\mathcal{K}^{\text {crit }}(Z)$ we note that

$$
2 P_{Z} \leq \Lambda_{Z} \leq \mu_{\max }(Z) P_{Z}
$$

where $\mu_{\max }(Z)$ is the maximum eigenvalue of $\Lambda_{Z}$. We recall here that $\Lambda_{Z}$ is the matrix of the Hodge metric (see (30) ), and its eigenvalues can be estimated in terms of the WeilPetersson metric and its curvature (cf. [Lu] ). In particular, its minimum eigenvalue satisfies $\mu_{\min }(Z) \geq 2$, and that explains the lower bound $2 P_{Z}$ in (126). For most CY 3 -folds $X$, the Weil-Petersson metric on $\mathcal{C}$ is incomplete, and $\mu_{\max }(Z) \rightarrow \infty$ as $Z$ tends to the boundary (Z. Lu).

By (126), we have

$$
J_{-}\left(\mu, P_{Z}\right) \leq\left(b_{3} !\right) \mathcal{K}^{\mathrm{crit}}(Z) \leq J_{+}\left(\mu, P_{Z}\right), \quad\left(\forall \mu \geq \mu_{\max }(Z)\right)
$$

where

$$
J_{+}\left(\mu, P_{Z}\right):=\left.\frac{1}{b_{3} / 2-1} \int_{\mathcal{H}_{Z} \oplus \mathbb{C}}\left|\operatorname{det} H^{*} H-\right| x\right|^{2} I \mid e^{-\left(\mu^{-1} \operatorname{Tr} H^{*} H-|x|^{2}\right)} d H d x
$$


and where

$$
J_{-}\left(\mu, P_{Z}\right):=\left.\frac{1}{\mu^{\left(b_{3} / 2-1\right)}} \int_{\mathcal{H}_{Z} \oplus \mathbb{C}}\left|\operatorname{det} H^{*} H-\right| x\right|^{2} I \mid e^{-\left(2^{-1} \operatorname{Tr} H^{*} H-|x|^{2}\right)} d H d x,
$$

Thus we obtain upper and lower bounds for the density in regions $K_{\mu} \subset \mathcal{C}$ for which the absolute values of the eigenvalues of the Hodge metric relative to the Weil-Petersson metric satisfy $\mu_{\max }(Z) \leq \mu$. We have bounded the determinant of $\Lambda$ by a power of an extremal eigenvalue, but it could also be identified with the volume density of the Hodge metric. We note that the lower bound tends to zero and the upper bound tends to infinity in $\sim \pm b_{3}$ powers of $\mu_{\max }(Z)$ as $Z \rightarrow \partial \mathcal{C}$ when the Weil-Petersson metric is incomplete and the norm of the Ricci curvature of $\omega_{W P}$ tends to infinity.

We now estimate $J_{ \pm}\left(\mu, P_{Z}\right)$ under the assumption that $\mathcal{H}_{Z}$ is a 'sufficiently random' subspace. The subspace $\mathcal{H}_{Z}$ is a real subspace of dimension $b_{3}-2$ of $\operatorname{Sym}\left(b_{3} / 2-1\right.$, $\left.\mathbb{C}\right)$, but by modifying the definition of the complex structure it becomes a complex $b_{3} / 2$-dimensional one. Hence, we may view $Z \rightarrow \mathcal{H}_{Z}$ as a map $\mathcal{C} \rightarrow \operatorname{Gr}\left(b_{3} / 2-1, \operatorname{Sym}\left(b_{3} / 2-1, \mathbb{C}\right)\right)$ to the complex Grassmannian of $b_{3} / 2-1$ dimensional complex subspaces. Lacking knowledge of the distribution of the image of $Z \rightarrow \mathcal{H}_{Z}$, we make the assumption that it is random, or more precisely we approximate $J_{ \pm}\left(\mu, P_{Z}\right)$ by the expected value of $J_{ \pm}(\mu, P)$, where $P$ is the projection corresponding to a random element $\mathcal{H} \in G r\left(b_{3} / 2-1, \operatorname{Sym}\left(b_{3} / 2-1, \mathbb{C}\right)\right)$.

This approximation by the expected value seems to be reasonable because Grassmannians $\operatorname{Gr}(k, N)$ are examples of Gromov-Milman 'Levy families' of Riemannian manifolds for which concentration of measure phenomena hold as $N \rightarrow \infty$ GM, Ta. Concentration of measure refers to a metric space $(X, d)$ with a probability measure $P$ and a concentration function $\alpha(P, t)$, which is the smallest number such that the measure of a set $A$ and the metric tube $A_{t}=\{x: d(x, A)<t\}$ around $A$ are related by $P(A) \geq 1 / 2 \Longrightarrow P\left(A_{t}\right) \geq 1-\alpha(P, t)$. If $f$ is a Lipschitz function and if $M_{f}$ is a median for $f$, we put $A=\left\{x: f(x) \leq M_{f}\right\}$, and then $P\left(\left|f-M_{f}\right|>t\right) \leq 2 \alpha\left(P, \frac{t}{\|f\|_{L i p}}\right)$. Concentration of measure occurs if $\alpha(P, t)$ decays rapidly in $t$, and thus $f$ is highly concentrated around its median. In a Lévy family $\left(X_{N}, d_{N}\right)$, the functions $\alpha_{N}(P, t)$ decay at ever faster rates depending on $N$. For instance on the unit $N$-sphere $S^{N}$, the rate is (a universal constant times) $e^{-\frac{(N-1)}{2} t^{2}}$.

In our setting, the family consists of Grassmannians $\operatorname{Gr}\left(b_{3} / 2-1, \operatorname{Sym}\left(b_{3} / 2-1, \mathbb{C}\right)\right)$ equipped with the invariant probability measure $d \nu$ and with the standard bi-invariant metric. It is pointed out in [GM] that $\operatorname{Gr}(k, N)$ is a Lévy family for fixed $k$ (see section (3.3) of [GM]), and the same argument should apply to $k_{N} \sim N / 2$. Moreover, $\{U(N)\}$ with its Haar probability measure and bi-invariant metric is Lévy, and by section (2.1) of [GM] its quotients should be. The function $f$ is $J_{ \pm}(\mu, P)$ for fixed $\mu$. Since we are mainly interested in factorial dependencies, we set $\mu=1$ and change the exponent $2^{-1}$ to 1 to make the Gaussian measure a probability measure. In general, the result would be modified by a $\pm b_{3}$ power of $\mu$. In this heuristic discussion, we will not attempt to determine $\alpha_{N}(P, t)$ or $M_{f}$ but will assume that $\alpha\left(P, \frac{t}{\|f\|_{\text {Lip }}}\right)$ has rapid decrease in $t$ which improves with the dimension. We also note that when $\alpha(P, t)$ is small, we can replace the median of $J_{ \pm}(\mu, P)$ (with $\left.\mu=1\right)$ by its mean

$$
\int_{\operatorname{Gr}\left(b_{3} / 2-1, \operatorname{Sym}\left(b_{3} / 2-1, \mathbb{C}\right)\right.}\left\{\int_{\mathcal{H} \oplus \mathbb{C}}\left|\operatorname{det}\left(H^{*} H-|x|^{2} I\right)\right| e^{-T r H^{*} H-|x|^{2}} d H d x\right\} d \nu(\mathcal{H})
$$


with a small error (cf. [Ta]). This mean equals

$$
\int_{\operatorname{Sym}\left(b_{3} / 2-1, \mathbb{C}\right) \oplus \mathbb{C}}\left|\operatorname{det}\left(H^{*} H-|x|^{2} I\right)\right| e^{-T r H^{*} H-|x|^{2}} d H d x
$$

since both measures are invariant probability measures and are therefore equal. Here we ignore factors of $(2 \pi)$ (etc.) for the sake of simplicity, since we are primarily interested in the factorially growing quantities. Due to the concentration of measure, the spaces $\mathcal{H}_{Z}$ would have to be very 'rare events' if $J_{ \pm}\left(\mu, P_{Z}\right)$ differed appreciably from its mean. We note that since $H_{Z}^{3}$ is a complex polarization, $P_{Z}$ has special features that do not hold for random subspaces, but we have no reason to believe that these special features bias $J\left(\mu, P_{Z}\right)$ away from its mean.

We now observe that (130) (with any choice of $\mu$ ) is similar to the integral for the density of critical points for holomorphic sections of $\mathcal{O}(N) \rightarrow \mathbb{C P}^{m}$ with $m=b_{3} / 2-1$ with respect to the Fubini-Study connection for a fixed degree $N$ [DSZ2] ( $\$ 4)$. There, the $\Lambda_{Z}$ matrix was (for every $Z$ ) a two-block diagonal matrix with a large scalar block and a $1 \times 1$ scalar block. When $\mu=1$ (130) agrees with that $\mathcal{O}(N) \rightarrow \mathbb{C P}^{m}$ density in the case $N=1$. As noted in DSZ2, the total number of critical points of a given Morse index appears to grow at a rate $N^{m}$ times a rational quantity in $m$ as $m \rightarrow \infty$. This growth rate may also be easily verified for the Euler characteristic $\chi\left(T^{* 1,0} \otimes \mathcal{O}(N)\right)$, i.e. the alternating sum over the Morse indices, which is given by

$$
\chi\left(T^{* 1,0} \otimes \mathcal{O}(N)\right)=\left(\frac{c(\mathcal{O}(N-1))^{m+1}}{c(\mathcal{O}(N))},\left[\mathbb{C P}^{m}\right]\right)=\frac{(N-1)^{m+1}+(-1)^{m}}{N} .
$$

Since the volume of $\mathbb{C P}^{m}$ is $\frac{1}{m !}$, this would imply that the density of critical points grows like $m$ ! with the dimension. On this basis, we would expect that $J_{ \pm}\left(\mu, P_{Z}\right)$ for $\mu \simeq 1$ grows with the dimension at the rate $\left(b_{3} / 2\right) ! N_{\mu}^{b_{3}}$ for some $N_{\mu}>0$.

We note that the Ashok-Douglas heuristic that the density of critical points should have the same order of magnitude as the index density is indeed correct in the setting of $\mathcal{O}(N) \rightarrow \mathbb{C P}^{m}$. Further, the origin of the factorials $\left(b_{3} / 2\right)$ ! is essentially in both the $\mathcal{C}$ and $\mathbb{C P}^{m}$ settings.

Thus our heuristics give $\mathcal{K}^{\text {crit }}(Z) \sim \frac{\left(b_{3} / 2\right) ! N_{\mu}^{b_{3}}}{b_{3} !}$. If we integrate over $K_{\mu}$ and apply the conjectural volume bound $\frac{1}{\left.\left(b_{3} / 2\right) !\right)}$ for $K_{\mu}$, we would get roughly $\frac{L^{b_{3}} N_{\mu}^{b_{3}}}{b_{3} !}$. Further applying the observed relation $L \sim C b_{3}$ with $C \in[1 / 3,3]$ gives an exponential growth rate for numbers of vacua in $K_{\mu}$.

\section{REFERENCES}

[AD] S. Ashok and M. R. Douglas, Counting Flux Vacua, JHEP 0401 (2004) 060 (hep-th/0307049).

[BGV] N. Berline, E. Getzler, and M. Vergne, Heat kernels and Dirac operators. Grundlehren der Mathematischen Wissenschaften 298. Springer-Verlag, Berlin, 1992.

[BSZ1] P. Bleher, B. Shiffman and S. Zelditch, Universality and scaling of correlations between zeros on complex manifolds, Invent. Math. 142 (2000) 2, 351-395.

[BSZ2] P. Bleher, B. Shiffman and S. Zelditch, Correlations between zeros and supersymmetry, Commun. Math. Phys. 224 (2001) 1, 255-269.

[BP] R. Bousso and J. Polchinski, Quantization of four-form fluxes and dynamical neutralization of the cosmological constant, J. High Energy Phys. 2000, no. 6, Paper 6.

[CHSW] P. Candelas, G. Horowitz, A. Strominger, and E. Witten, Vacuum configurations for superstrings. Nuclear Phys. B 258 (1985), no. 1, 46-74. 
[CO] P. Candelas, X. C. de la Ossa, Moduli space of Calabi-Yau manifolds. Nuclear Phys. B 355 (1991), no. 2, 455-481; also appeared in Strings '90 (College Station, TX, 1990), 401-429, World Sci. Publishing, River Edge, NJ, 1991.

[DD1] F. Denef and M. R. Douglas, Distributions of flux vacua, J. High Energy Phys. 2004, no. 5, 072 (hep-th/0404116).

[DD2] F. Denef and M. R. Douglas, Distributions of nonsupersymmetric flux vacua, hep-th/0411183.

[DGKT] O. DeWolfe, A. Giryavets, S. Kachru and W. Taylor, Enumerating Flux Vacua with Enhanced Symmetries (hep-th/0411061).

[Do] M. R. Douglas, The statistics of string/M theory vacua. J. High Energy Phys. 2003, no. 5, 046 (hep-th/0303194).

[DSZ1] M. R. Douglas, B. Shiffman and S. Zelditch, Critical Points and supersymmetric vacua I, Comm. Math. Phys. 252 (2004), no. 1-3, 325-358.

[DSZ2] M. R. Douglas, B. Shiffman and S. Zelditch, Critical Points and supersymmetric vacua II: Asymptotics.

[DO] W. Duke and O. Imamoglu, Lattice points in cones and Dirichlet series, IMRN 53 (2004).

[Fy] Y. V. Fyodorov, Complexity of Random Energy Landscapes, Glass Transition and Absolute Value of Spectral Determinant of Random Matrices Physical Review Letters v. 92 (2004), 240601; Erratum: ibid. v. 93 (2004), 149901 (cond-mat/0401287).

[GKP] S.B. Giddings, S. Kachru, and J. Polchinski, Hierarchies from fluxes in string compactifications. Phys. Rev. D (3) 66 (2002), no. 10, 106006.

[GKT] A. Giryavets, S. Kachru and P. K. Tripathy, On the taxonomy of flux vacua, JHEP 0408:002, 2004 (hep-th/0404243).

[GH] P. Griffiths and J. Harris, Principles of Algebraic Geometry, Wiley-Interscience, New York (1978).

[GM] M. Gromov and V. D. Milman, A topological application of the isoperimetric inequality. Amer. J. Math. 105 (1983), no. 4, 843-854.

[GHJ] M. Gross, D. Huybrechts, and D. Joyce, Calabi-Yau Manifolds and Related Geometries, Springer Universitext, Springer, New York (2003).

[GVW] S. Gukov, C. Vafa, and E. Witten, CFT's from Calabi-Yau four-folds. Nuclear Phys. B 584 (2000), no. $1-2,69-108$.

[HW] G. H. Hardy and E. M. Wright, An introduction to the theory of numbers. Fifth edition. The Clarendon Press, Oxford University Press, New York, 1979.

[Hl] E. Hlawka, Über Integrale auf konvexen Körpern I, II, Monatsh. Math. 54 (1950), 1-36, 81-99.

$[\mathrm{Ho}] \quad$ L. Hörmander, The analysis of linear partial differential operators. I. Distribution theory and Fourier analysis. Classics in Mathematics. Springer-Verlag, Berlin, 2003.

[KKLT] S. Kachru, R. Kallosh, A. Linde and S. P. Trivedi, De Sitter vacua in string theory, Phys. Rev. D 68, 046005 (2003), (hep-th/0301240).

[KL] R. Kallosh and A. Linde, Landscape, the scale of SUSY breaking, and inflation, hep-th/0411011.

[KLRY] A. Klemm, B. Lian, S. S. Roan and S. T. Yau, Calabi-Yau fourfolds for M- and F-theory compactifications, Nucl. Phys. B 518, 515 (1998) (arXiv:hep-th/9701023).

[Lu] Z. Lu, On the Hodge metric of the universal deformation space of Calabi-Yau threefolds. J. Geom. Anal. 11 (2001), no. 1, 103-118.

[LS1] Z. Lu and X. Sun, On the Weil-Petersson volume and the first Chern Class of the moduli space of Calabi-Yau manifolds, math.DG/0510021

[LS2] Z. Lu and X. Sun, Weil-Petersson geometry on moduli space of polarized Calabi-Yau manifolds, J. Inst. Math. Jussieu (2004) 3(2), 185-229 (math.DG/0510020).

[NR] M. Nechayeva and B. Randol, Approximation of measures on $S^{n}$ by discrete measures (preprint, 2005).

[Po] Ch. Pommerenke, Über die Gleichverteilung von Gitterpunkten auf $m$-dimensionalen Ellipsoiden. Acta Arith. 5 (1959), 227-257.

[Ra] B. Randol, A lattice-point problem. Trans. Amer. Math. Soc. 121 (1966), 257-268. 
[Si] E. Silverstein, AdS and dS Entropy from String Junctions, in From Fields to Strings: Circumnavigating Theoretical Physics, Ian Kogan Memorial collection, vol. 3* Shifman, M. (ed.), World Scientific, pp. 1848-1863 (hep-th/0308175).

[St1] A. Strominger, Special geometry. Comm. Math. Phys. 133 (1990), no. 1, 163-180.

[St2] A. Strominger, Kaluza-Klein compactifications, supersymmetry and Calabi-Yau manifolds, in Quantum fields and strings: a course for mathematicians, P. Deligne et. al., eds., American Mathematical Society, Providence, RI; Institute for Advanced Study (IAS), Princeton, NJ, 1999, Vol. 2, pp. 1091-1115.

[Sul] D. Sullivan, Infinitesimal computations in topology. Inst. Hautes Études Sci., Publ. Math. No. 47, (1977), 269-331 (1978).

[Sus] L. Susskind, The anthropic landscape of string theory, hep-th/0302219.

[Ta] Michel Talagrand, A new look at independence, Ann. Probab. 24 (1996), no. 1, 1-34.

[Wa1] C.-L. Wang, On the incompleteness of the Weil-Petersson metrics along degenerations of CalabiYau manifolds, Math. Res. Lett. 1 (1997), 157-171.

[Wa2] C.-L. Wang, Curvature properties of the Calabi-Yau moduli, Doc. Math. 8 (2003), 577-590.

[WB] J. Wess and J. Bagger, Supersymmetry and supergravity. Second edition. Princeton Series in Physics. Princeton University Press, Princeton, NJ, 1992.

[Ze1] S. Zelditch, Angular distribution of lattice points (in preparation).

[Ze2] S. Zelditch, Random complex geometry and vacua, or: How to count universes in string/M theory (preprint, 2005).

NhetC and Department of Physics and Astronomy, Rutgers University, Piscataway, nJ 08855-0849, USA; And I.H.E.S., Bures-Sur-Yvette, France

E-mail address: mrd@physics.rutgers.edu

Department of Mathematics, Johns Hopkins University, Baltimore, MD 21218, USA

E-mail address: shiffman@math.jhu.edu

Department of Mathematics, Johns Hopkins University, Baltimore, MD 21218, USA

E-mail address: zelditch@math.jhu.edu 